\title{
Towards personalized robot-assisted gait training
}

Simone Fricke 


\section{TOWARDS PERSONALIZED ROBOT-ASSISTED GAIT TRAINING}

Simone Sarah Fricke 



\title{
TOWARDS PERSONALIZED ROBOT-ASSISTED GAIT TRAINING
}

\section{DISSERTATION}

\author{
to obtain \\ the degree of doctor at the University of Twente, \\ on the authority of the rector magnificus, \\ prof.dr. T.T.M. Palstra, \\ on account of the decision of the Doctorate Board, \\ to be publicly defended \\ on Friday 10 July 2020 at 12.45
}

by

Simone Sarah Fricke

born on 20 July 1990

in Bremen, Germany 
This dissertation has been approved by:

Supervisor:

prof.dr.ir. H. van der Kooij

Co-supervisor:

dr. E.H.F. van Asseldonk

Cover design: Milou Mulder

Printed by: Ipskamp printing

ISBN: 978-90-365-5002-4

DOI: $10.3990 / 1.9789036550024$

(C) 2020 Simone Sarah Fricke, Enschede, The Netherlands. This dissertation is published under the terms of the Creative Commons AttributionNonCommercial 4.0 International License, which permits unrestricted use, distribution, and reproduction in any medium, provided the original work is properly credited and any changes to the used material are indicated. 


\section{GRADUATION COMMITTEE}

Chairman/secretary

prof.dr.ir. H.F.J.M. Koopman

University of Twente

\section{Supervisor}

prof.dr.ir. H. van der Kooij

University of Twente

Co-supervisor

dr. E.H.F. van Asseldonk

University of Twente

Committee members

prof.dr. A.C.H. Geurts

Radboud University

dr.ir. J.C. Moreno

Spanish National Research Council

dr. A.R. den Otter

University of Groningen

prof.dr. J.S. Rietman

University of Twente

dr. E. Swinnen

Vrije Universiteit Brussel

prof.dr.ir. P.H. Veltink

University of Twente 
This work is part of the research programme AWARD with project number 12850, which is financed by the Netherlands Organisation for Scientific Research (NWO).

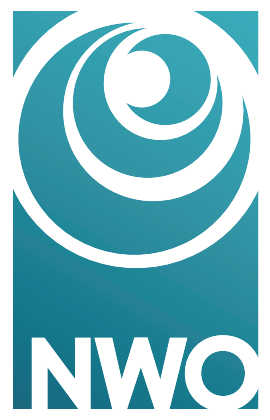




\section{Contents}

\begin{tabular}{lr}
\hline Summary & 9
\end{tabular}

$\begin{array}{ll}\text { Samenvatting } & 11\end{array}$

$\begin{array}{lll}1 & \text { General introduction } & 15\end{array}$

1.1 Robot-assisted gait training . . . . . . . . . . . . . . . . . 16

1.2 Robot-assisted assessment of sensorimotor impairments and gait . 24

1.3 Thesis objectives and outline $\ldots \ldots \ldots \ldots \ldots \ldots$

References....................... 26

2 Design and evaluation of a lower limb perturbator to estimate hip $\begin{array}{ll}\text { joint impedance during walking } & 41\end{array}$

2.1 Introduction . . . . . . . . . . . . . . . . . . . . . . 42

2.2 Requirements . . . . . . . . . . . . . . . . . . . . 42

2.3 Methods . . . . . . . . . . . . . . . . . . . . . . . . . 43

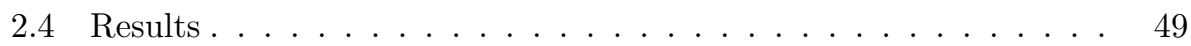

2.5 Discussion . . . . . . . . . . . . . . . . . . . . . . . . . . 52

2.6 Conclusion $\ldots \ldots \ldots \ldots \ldots$

References ............................ 56

3 Performance-based adaptive assistance for diverse subtasks of walking in a robotic gait trainer: Description of a new controller $\begin{array}{ll}\text { and preliminary results } & 61\end{array}$

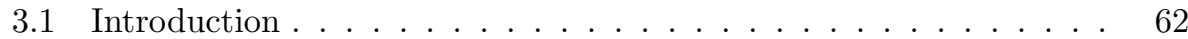

3.2 Methods . . . . . . . . . . . . . . . . . . . . . . . . 63

3.3 Results . . . . . . . . . . . . . . . . . . . . . . 66

3.4 Discussion and conclusion . . . . . . . . . . . . . . . . . . . . . . 69

References.............................. 71

4 Automatic versus manual tuning of robot-assisted gait training $\begin{array}{ll}\text { in people with neurological disorders } & 73\end{array}$

4.1 Introduction $\ldots \ldots \ldots \ldots \ldots \ldots \ldots \ldots$

4.2 Methods . . . . . . . . . . . . . . . . . . . . . . . . . . 75

4.3 Results . . . . . . . . . . . . . . . . . . . . . . . . . . . . . . . . . . . 84

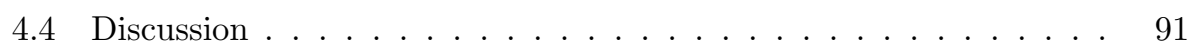

4.5 Conclusion $\ldots \ldots \ldots \ldots \ldots$ 


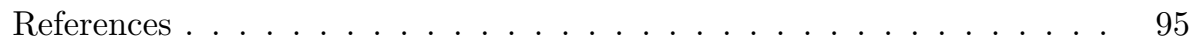

\begin{tabular}{|lll|}
5 & Effects of selectively assisting impaired subtasks of walking in \\
\hline & chronic stroke survivors & 101
\end{tabular}

5.1 Introduction . . . . . . . . . . . . . . . . . . . . 102

5.2 Methods . . . . . . . . . . . . . . . . . . . 103

5.3 Results . . . . . . . . . . . . . . . . . . . . . . . . 110

5.4 Discussion . . . . . . . . . . . . . . . . 115

5.5 Conclusion . . . . . . . . . . . . . . . . . . . . . . 119

References . . . . . . . . . . . . . . . . . . 119

$\begin{array}{lll}6 & \text { General discussion } & 123\end{array}$

6.1 Major findings and implications . . . . . . . . . . . . . . . . . . . 124

6.2 Future steps and recommendations . . . . . . . . . . . . . . . . . . 129

6.3 Final remarks . . . . . . . . . . . . . . . . . . . . . 131

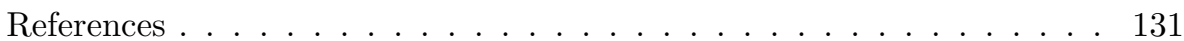

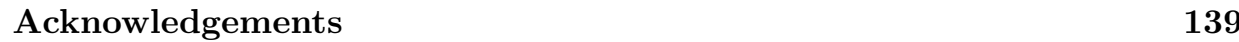

\begin{tabular}{ll}
\hline Biography & 141
\end{tabular}

\begin{tabular}{ll}
\hline Scientific contributions & 143
\end{tabular} 


\section{Summary}

Robot-assisted gait training (RAGT) is a promising tool to improve walking function after stroke and spinal cord injury (SCI), especially when combined with conventional physical therapy. The way how the robot is controlled can have a large influence on active participation of the user and the effectiveness of the training. Previous studies suggest that personalized assistance based on patients' abilities can be beneficial as it can increase active participation of the user. Besides, robotic gait trainers cannot only provide training, but also have the potential to be used for assessment of walking function and impairments affecting walking function. The goal of this thesis is to take a next step towards personalized robot-assisted gait training by 1 . developing assessment tools to quantify walking function and underlying impairments affecting walking function, and 2. improving subtask-based assistance and optimizing assistance tuning based on users' walking abilities.

Knowledge about the mechanical properties of the joints (i.e. joint impedance) can be used to monitor patients' progress, improve training protocols and adjust controllers for RAGT. While ankle joint impedance during walking has been determined in previous studies, hip joint impedance during walking is unknown. The goal of Chapter 2 is to develop and evaluate a device that can be used to determine hip joint impedance during walking, and to get first estimates of apparent hip joint impedance during walking. We developed the LOPER (LOwer limb PERturbator) which has negligible effects on the walking pattern when attached to the upper leg, and can apply force perturbations during swing phase. Through application of perturbations at different instances of the swing phase, we were able to get first estimates of the time-varying behaviour of the apparent hip joint impedance during walking in healthy participants. In the future, the device and applied estimation methods should be evaluated in people with neurological disorders. In addition, the device and estimation methods should be extended to the knee joint to be able to estimate knee joint impedance and possible interactions between knee and hip joint impedance.

The minimal assistance that a user needs to walk in a robotic gait trainer can be a measure of the user's walking function. The lower this assistance is, the better the walking function. Controllers that automatically adjust assistance based on users' performance could be used as assessment tools for walking function. However, results from current automatically-tuned (AT) algorithms can be difficult to interpret, as assistance is adjusted for several intervals of the gait cycle which are not directly related to functional aspects of gait. In addition, it is not known how the applied assistance is affected by changes in training parameters, such as partial 
body weight support (PBWS) and walking speed, that can occur during therapy. In Chapter 3, we describe a controller that automatically adjusts the assistance for various subtasks of walking (e.g. foot clearance, stability during stance) based on users' performance. The effects of changes in PBWS and walking speed on the applied assistance and subtask performance were evaluated. Experiments in ten healthy participants showed that subtask performance, and thus applied assistance, was influenced by both, PBWS and walking speed. The applied assistance by the AT subtask-based controller can be used as an assessment tool of walking function, but only if PBWS and walking speed are kept constant.

Manual tuning of subtask-based assistance can be difficult, time-intensive and depends on subjective decisions of therapists. Automatic assistance tuning can tackle these problems, but potentially has drawbacks, too. The goal of Chapter 4 is to determine differences and similarities between manually- and automaticallytuned (MT and AT) subtask-based assistance in people with neurological disorders (stroke or SCI). Participants' preferences, time to tune the assistance, final assistance levels and errors compared to reference trajectories were analyzed. Participants did not prefer one approach (AT or MT) over the other regarding comfort, safety, and amount and effect of applied assistance. We found several advantages of AT assistance compared to MT assistance in people with neurological disorders who were walking in the LOPES II gait trainer: quicker assistance tuning, lower assistance levels and separate tuning of each subtask resulting in a good performance for all subtasks. Clinical trials are needed to show whether these apparent advantages of AT assistance also result in better clinical outcomes.

In Chapters 3 and 4 , automatic assistance tuning was performed for all subtasks simultaneously. We did not consider whether assisting one subtask (e.g. the most impaired one) would also affect other subtasks. In Chapter 5, we aimed to get a better understanding of separate assistance for the most impaired subtasks of walking after stroke: foot clearance (FC), stability during stance (SS) and weight shift (WS). Performance for the impaired, assisted subtasks clearly improved in mildly impaired chronic stroke survivors compared to walking in LOPES II without assistance. Our WS assistance can be further optimized so that users shift the weight better towards the paretic leg. Performance improvements for the assisted subtasks only rarely resulted in more general changes of the walking pattern, i.e. effects on other subtasks or spatiotemporal parameters. Therefore, in mildly impaired stroke survivors, assistance for various subtasks of walking can be tuned simultaneously resulting in quick assistance tuning. There is no need for specific, time-intensive, tuning protocols in these patients, such as tuning subtasks after each other while starting with the most impaired subtask.

To sum up, we developed assessment methods and a control algorithm that automatically adjusts subtasks-based assistance based on users' performance. We have taken a next step towards personalized robot-assisted gait training. In the discussion we show that there are still some challenges to overcome in order to apply the optimal robotic gait assistance for each individual. Future research should focus on the long-term effect of various controllers and a better understanding of the exact effect of RAGT on neurorehabilitation after stroke and SCI to further personalize and improve RAGT. 


\section{Samenvatting}

Robotisch ondersteunde looptraining is een veelbelovende training voor het verbeteren van de loopfunctie na een beroerte of dwarslaesie, vooral in combinatie met conventionele fysiotherapie. De manier waarop de robot aangestuurd wordt kan een grote invloed hebben op de eigen activiteit van de patiënt en de effectiviteit van de training. Eerdere studies suggereren dat gepersonaliseerde ondersteuning op basis van de vaardigheden van de patiënt voordelig kan zijn. Bovendien kunnen looprobots niet alleen voor de training gebruikt worden, maar ze zouden ook gebruikt kunnen worden voor het meten van de loopfunctie of beperkingen die de loopfunctie beïnvloeden. Het doel van dit proefschrift is om een volgende stap te zetten naar gepersonaliseerde robotisch ondersteunde looptraining door 1 . het ontwikkelen van meetmethoden voor de loopfunctie en beperkingen die de loopfunctie beïnvloeden, en 2 . het verbeteren van subtaak-gebaseerde ondersteuning en het optimaliseren van het instellen van de ondersteuning op basis van de loopcapaciteiten van de patiënt.

Kennis over de mechanische eigenschappen van gewrichten (i.e. gewrichtimpedantie) kan gebruikt worden om de voortgang van patiënten te volgen, oefenprotocollen te verbeteren en aansturingsmechanismen voor robotisch ondersteunde looptraining aan te passen. De enkel-impedantie is al in eerdere studies bepaald, maar de heup-impedantie is onbekend. Het doel van Hoofdstuk 2 is om een apparaat te ontwikkelen en te beoordelen dat gebruikt kan worden om de heup-impedantie tijdens het lopen te meten, en om een eerste schatting van de heup-impedantie te maken. We hebben de LOPER (LOwer limb PERturbator) ontwikkeld die verwaarloosbare effecten op de loopcyclus heeft als die aan het bovenbeen is vastgemaakt, en die krachtverstoringen tijdens de zwaaifase kan aanbrengen. Door het aanbrengen van verstoringen op verschillende tijdstippen tijdens de zwaaifase, hebben we eerst schattingen van het tijdsvariante gedrag van de heup-impedantie tijdens het lopen in gezonde proefpersonen gekregen. In de toekomst zouden het apparaat en de gebruikte schattingsmethoden in proefpersonen met neurologische aandoeningen geëvalueerd moeten worden. Daarnaast moeten het apparaat en de schattingsmethodes uitgebreid worden naar het kniegewricht om knie-impedantie en mogelijke interacties tussen knie- en heup-impedantie te kunnen schatten.

De minimale ondersteuning die een gebruiker nodig heeft om in een robotische looptrainer te lopen zou een maat voor de loopfunctie kunnen zijn. Hoe lager deze ondersteuning is, hoe beter de loopfunctie. Aansturingsmechanismen die automatisch de ondersteuning aanpassen gebaseerd op de prestaties van de gebruiker 
zouden als meetinstrumenten voor de loopfunctie gebruikt kunnen worden. Uitkomsten van eerdere studies met een automatisch aangepaste ondersteuning zijn moeilijk te interpreteren omdat de ondersteuning voor een aantal intervallen van de loopcyclus, die niet aan functionele taken gerelateerd zijn, wordt aangepast. Daarnaast is onbekend hoe de ondersteuning beïnvloedt wordt door veranderingen in oefenparameters, zoals de mate van gewichtsondersteuning of loopsnelheid, die tijdens de therapie kunnen optreden. In Hoofdstuk 3 beschrijven we een aansturingsmechanisme dat automatisch de ondersteuning voor diverse subtaken van het lopen (bijv. creëren van voldoende ruimte tussen de teen en de grond tijdens de zwaaifase ('foot clearance'), stabiliteit tijdens de standfase) aanpast op basis van de prestaties van de gebruiker. We hebben het effect van veranderingen van de gewichtsondersteuning en loopsneldheid op de toegepaste ondersteuning en prestaties voor verschillende subtaken beoordeeld. Metingen in tien gezonde proefpersonen lieten zien dat prestaties voor verschillende subtaken, en dus ook de hoeveelheid ondersteuning, afhankelijk waren van beide oefenparameters (loopsnelheid en gewrichtsverplaatsing). De toegepaste ondersteuning van het automatische subtaak-gebaseerde aansturingsmechanisme kan als een meetinstrument gebruikt worden, maar alleen als de gewichtsondersteuning en loopsnelheid constant zijn.

Het handmatige aanpassen van de op subtaken gebaseerde ondersteuning kan moeilijk en tijdrovend zijn, en is afhankelijk van subjectieve beslissingen van therapeuten. Een automatische aanpassing van de ondersteuning kan deze problemen voorkomen, maar heeft mogelijk andere nadelen. Het doel van Hoofdstuk 4 is om handmatig en automatisch aangepaste ('manually-tuned' en 'automatically-tuned', MT en AT) op subtaken gebaseerde ondersteuning te vergelijken in proefpersonen met neurologische aandoeningen (beroerte en dwarslaesie). We hebben de voorkeuren van de proefpersonen, de tijd om de ondersteuning aan te passen, uiteindelijke niveaus van ondersteuning en fouten ten opzichte van referentiepatronen geanalyseerd. Wat betreft het comfort, de veiligheid, de hoeveelheid ondersteuning en het effect van de ondersteuning, hadden proefpersonen geen voorkeur voor één van de manieren (MT of AT). We hebben een aantal voordelen van de AT ondersteuning vergeleken met de MT ondersteuning in proefpersonen met neurologische aandoeningen gevonden die in de LOPES II looptrainer liepen: snellere aanpassing van de ondersteuning, lagere ondersteuningsniveaus, en de ondersteuning werd voor elke subtaak apart aangepast wat leidde tot goede prestaties voor alle subtaken. Klinische studies zijn nodig om te laten zien of deze mogelijke voordelen van de AT ondersteuning ook tot betere klinische uitkomsten leiden.

In Hoofdstuk 3 en 4 werd de ondersteuning voor alle subtaken tegelijkertijd automatisch aangepast. We hebben niet onderzocht of ondersteuning voor één subtaak (bijv. de meest aangedane subtaak) ook andere subtaken beïnvloedt. Het doel van Hoofdstuk 5 is om beter te begrijpen wat er gebeurt als de meest aangedane subtaken (foot clearance (FC), stabiliteit tijdens de standfase (SS) en gewichtsverplaatsing ('weight shift' (WS)) apart ondersteund worden. De prestaties voor de aangedane, ondersteunde subtaken waren aanzienlijk verbeterd vergeleken met lopen zonder ondersteuning in licht aangedane proefpersonen die een beroerte hebben gehad. Onze WS ondersteuning kan verder verbeterd worden zodat proefpersonen hun gewicht beter naar het paretische been verplaatsen. Verbeteringen 
van de prestaties voor de ondersteunde subtaken leidden slechts zelden tot algemene veranderingen van het looppatroon, i.e. effecten op andere subtaken of spatiotemporele parameters. Dat betekent dat in licht aangedane patiënten met een beroerte de ondersteuning voor meerdere subtaken tegelijkertijd aangepast kan worden. Dit leidt tot een snelle aanpassing van de ondersteuning. Het is niet nodig om in deze patiënten andere, tijdrovende protocollen voor het aanpassen van de ondersteuning te gebruiken, zoals subtaken na elkaar aanpassen terwijl er met de meest aangedane subtaak wordt begonnen.

Samenvattend, we hebben meetinstrumenten en een aansturingsmechanisme dat automatisch op subtaken gebaseerde ondersteuning aanpast afhankelijk van de prestaties van de gebruiker ontwikkeld. We hebben de volgende stap gezet in de richting van gepersonaliseerde robotisch ondersteunde looptraining. In de discussie laten we zien dat er nog steeds een aantal uitdagingen zijn die overwonnen moeten worden om voor iedereen de optimale robotische ondersteuning te kunnen bieden. Toekomstige onderzoeken zouden gericht moeten zijn op langetermijneffecten van verschillende aansturingsmechanismen en een beter begrip van het exacte effect van robotisch ondersteunde looptraining op de neurorevalidatie na een beroerte of dwarslaesie om de training verder te kunnen personaliseren en verbeteren. 


\section{Chapter 1}

\section{General introduction}

Walking is a common activity of daily living. For most people walking happens 'automatically'. Therefore, many people are not aware that walking is a highly complex activity, depending on various systems like the musculoskeletal, nervous and cardiovascular systems [1]. Unfortunately, since walking is such a complex activity, it is also prone to disturbances resulting in a diminished ability to walk or even complete immobility. This can have devastating consequences like a decreased quality of life, fear of falling and loss of independence 2 - 4 .

Stroke and spinal cord injury (SCI) are two disorders of the central nervous system (CNS) that commonly result in a diminished walking ability. A stroke occurs mainly in elderly. There are more than 400000 stroke survivors in the Netherlands and this number is expected to increase in the coming years [5]. A stroke is the result of either a rupture of a vessel in the brain (hemorrhagic stroke) or a blockage of a vessel (ischemic stroke) [6, [7]. In both cases, parts of the brain get damaged due to a lack of oxygen and nutrients, resulting in sensorimotor impairments (e.g. spasticity, muscle weakness, lack of muscle control), mostly on one side of the body, and problems during walking.

SCI can occur at any level of the spinal cord. SCI is often the result of a trauma (e.g. due to an accident), but can, for example, also be caused by diminished blood flow to the spinal cord or a tumor 8. Each year, about 240 people suffer a traumatic SCI in the Netherlands 9]. No reliable numbers are available about incidence of non-traumatic SCI, and prevalence of SCI 9]. Damage of the spinal cord distorts the communication between the central and peripheral nervous system which can again result in sensorimotor impairments (e.g. spasticity, muscle weakness, lack of muscle control) and problems during walking. The exact impairments depend on the location of the injury and the severity (complete/incomplete).

Recovery of gait is an important therapy goal after stroke and SCI [10 - 12]. Robot-assisted gait training (RAGT) has been developed to reduce physical load for therapists, and to provide people with stroke and SCI with intensive and taskspecific training. RAGT can result in effective training, especially when combined with conventional physical therapy [13, 14]. Still, therapy outcomes of RAGT could be further improved by increasing active participation of the user. This could be achieved by adjusting the assistance based on patients' impairments and 
performance during training $[15]-[17$.

This thesis describes four studies that were performed to improve RAGT by taking a next step towards personalized robot-assisted gait training. In the introduction, we present previous studies and controllers for RAGT. Subsequently, we focus on robot-assisted assessment of walking and underlying impairments affecting walking functions. Lastly, we present the goals and outline of this thesis.

\subsection{Robot-assisted gait training}

A number of principles to enhance recovery after stroke or SCI by common treatments can also be included into RAGT [17, [18. First, robotic gait trainers can be used to apply task-specific training. Task-specific training means that a complete task (e.g. walking) is practised, and training is not only based on impairments (e.g. muscle strength) or other tasks (e.g. standing). This task-specific training can lead to plastic changes in the CNS facilitating recovery after stroke and SCI [19-25]. Second, robotic gait trainers can provide training at high dosages which is beneficial for neurorehabilitation 18, 26], 27. Third, sensory feedback is crucial for learning. Robotically-guided movements result in sensory feedback from joint and load receptors [28. The lower the amount of partial body weight support (PBWS) during walking in robotic devices, the larger the amount of sensory feedback. Fourth, RAGT can be combined with feedback (e.g. auditory or haptic, or visual feedback based on virtual reality) which can further improve recovery 29 . Fifth, gait variability and active participation are crucial to (re)learn walking [17], 30]- 32]. However, as discussed later in this thesis (Section 1.1.2), this gait variability and active participation can be further optimized for RAGT.

RAGT has several advantages compared to previously used therapies, such as manually-assisted body weight supported treadmill training (i.e. where therapists manually guide movements of the patients) [17. RAGT largely reduces physical load for therapists because therapists do not have to move the legs of the patients manually when using RAGT 14. In addition, training protocols depend less on therapists' physical capacities and longer training sessions are possible [17], 33. Robotic gait trainers can provide full assistance which means that training sessions in severely affected patients can start earlier after stroke/SCI. Training sessions that were stopped in the past when therapists were fatigued, can continue and result in more intensive training for patients. Furthermore, sensors integrated in training devices allow for objective assessments during training. These assessments can be used to quantify impairments during walking (Section 1.2).

The following sections describe results from clinical studies and especially discuss previously implemented control algorithms for RAGT and possible improvements.

\subsubsection{Effect of RAGT on walking ability after stroke and SCI}

Reviews of several studies in stroke and SCI patients (including in total more than 1000 patients) have shown positive effects of RAGT compared to conventional gait training, but mainly when RAGT was combined with conventional gait training 
[13], [14], 34]-37]. The probability of independent walking was higher [13], and a higher walking speed and FAC (functional ambulation category [38]) [14] were found in stroke survivors who received combined training (RAGT and conventional therapy) compared to conventional training only. The largest improvements were seen in participants who received RAGT in the first three months after stroke and in participants who were not able to walk at the beginning of the therapy 13 .

Although reviews indicate that RAGT combined with conventional therapy is more effective than conventional therapy alone, results should be interpreted with caution. It cannot be assumed that RAGT always has beneficial effects due to possibly large differences between studies 13 . Several factors as different patient populations (e.g. severity, location of stroke/SCI), robotic gait trainers (e.g. end effector, exoskeleton, differences in transparency and degrees of freedom), training protocols (e.g. number and duration of training sessions), control algorithms and outcome measures were used [13], 14], 34. Especially differences in control algorithms (and the respective assistance that is applied) can have a crucial effect on active user participation and gait variability [39], influencing the effectiveness of RAGT as described in the following section.

\subsubsection{Control algorithms for RAGT}

Various control algorithms have been developed to apply assistance in a robotic device 15]. In this thesis, we focus on algorithms that were developed for exoskeleton type robotic gait trainers (e.g. Lokomat, LOPES II (Figures 1.1 and 1.2). The first developed control algorithms for robotic gait trainers were based on reference trajectories (Figure 1.3). Reference trajectories were derived from healthy participants [40], 41], determined based on movements of the less impaired leg of the patient [42], or by measuring movements of the impaired leg while a therapist manually assisted movements of the patient [43]. Other, more recent, control algorithms are based on neuromechanical models [4], [45] or apply pulses at specific parts of the gait cycle [46] (Figure 1.3). However, so far, no extensive clinical studies have been performed to show the effectiveness of these more recent controllers in people with stroke or SCI. Most of the previous research was related to controllers that use reference trajectories, and we focus on these reference trajectory based controllers in the following paragraphs.

Assistive controllers that require reference trajectories can be subdivided into the following two groups: position control and impedance control (Figure 1.3). Position control means that the patient is moved along reference trajectories by the device. This (passive) movement therapy is only suitable for severely or completely disabled patients. They are provided with sensory feedback and taskspecific training, but they do not have to move their legs themselves [17. These position-controlled devices do not require active participation of the user as the legs are moved by the device. With this guidance, patients with residual motor capacities are not sufficiently challenged and they will generate less muscle activity, which is also called 'slacking' 41], [47]. Training in a position-controlled device thus is not suitable for patients with residual movement capabilities.

Impedance control has been developed to increase active participation 


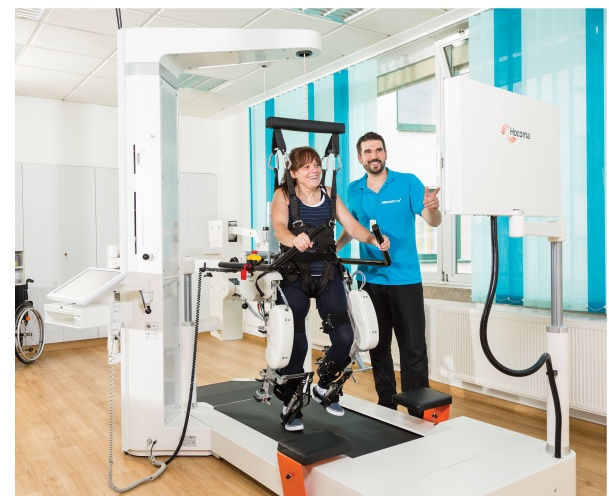

Figure 1.1: The Lokomat gait trainer. The device can actively assist the hip and knee joints in the sagittal plane during walking. Toes can be passively lifted with toelifters. The FreeD module allows for active assistance of lateral translation and transverse rotation of the pelvis, hip abduction is passively guided. Picture: Hocoma, Switzerland

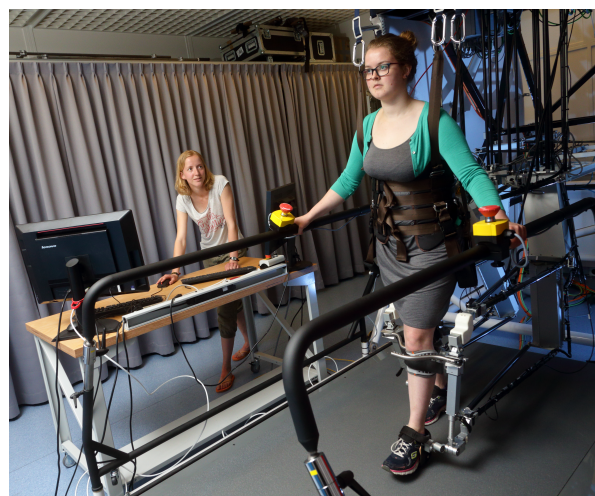

Figure 1.2: The LOPES II gait trainer. The pelvis (mediolateral, anteroposterior), hip (sagittal and frontal plane) and knee (sagittal) can be actively assisted. Toes can be passively lifted with toelifters. Rotation of the pelvis is possible in all planes. Picture: Gijs van Ouwekerk, Enschede, The Netherlands

and movement variability in patients with residual motor capacities. The torques/forces that are applied by impedance-based controllers depend on deviations from reference trajectories 39. The larger the deviation from reference trajectories, the higher the torques/forces that move the user towards the reference trajectories. Several variations of impedance-based controllers for RAGT have been developed and were used in previous studies (Figure 1.4). Although this list might not be exhaustive, the following aspects often differed between controllers: allowed variability, assistance timing, tunable parameters, assistance tuning and formulation. For each of these aspects various options are shown in Figure 1.4 Different options for these aspects can be combined in a specific controller.

A disadvantage of the first impedance-based controllers was that they did not allow for much spatial and temporal variability during gait [48. To increase active participation and gait variability during RAGT, several variations of impedancebased controllers were developed (see 'Allowed variability' in Figure 1.4). To allow for variability in timing, algorithms which take into account the preferred cadence of the user can be used [49], [50]. These algorithms check whether the user advances or runs behind the reference trajectory. Based on this, the replay speed of the reference trajectory is increased or decreased. This temporal variability was implemented in the control of devices such as LOPES I and II 51, 52 as well as the Lokomat [50]. To allow for spatial gait variability, a 'deadband' can be included around the reference trajectories [50], [53]-55]. Free movements are allowed within this deadband and assistance is only applied outside of the deadband. This deadband has already been implemented in the control of devices 


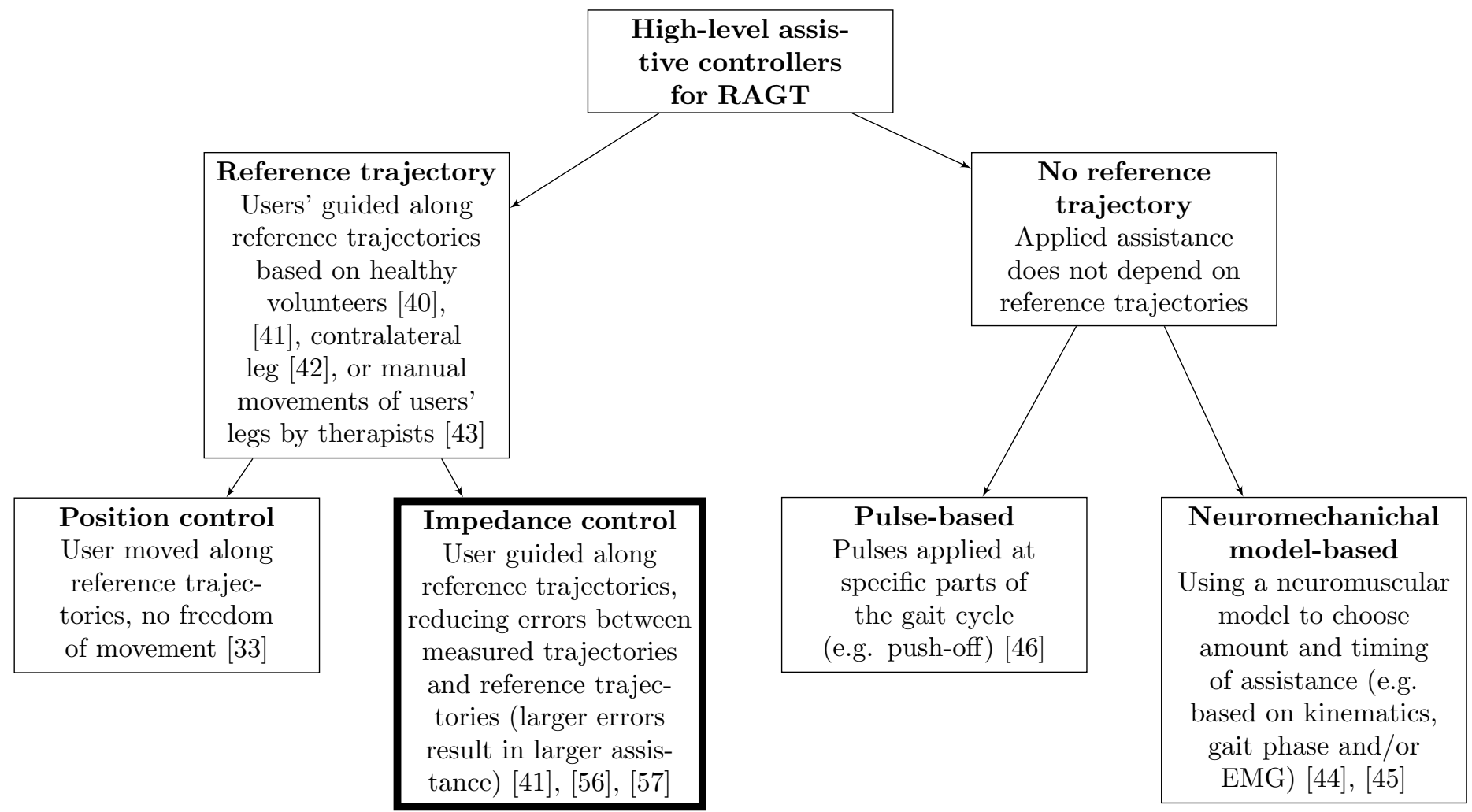

Figure 1.3: Overview of several high-level controllers that can be used for assisting patients during RAGT. Previous studies mostly focused on impedance-based controllers. Several variations of these impedance-based controllers are illustrated in Figure 1.4 
Allowed variability Assistance timing

\begin{tabular}{|c|}
\hline No spatial or \\
temporal variability \\
Assistance for all \\
deviations, replay \\
speed reference tra- \\
jectory not adjusted \\
automatically 48$]$ \\
\hline
\end{tabular}

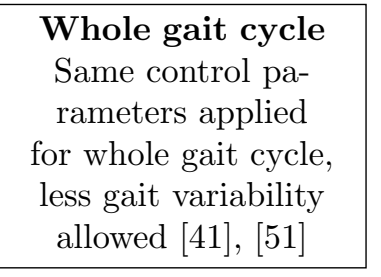

\section{Specific parts \\ of gait cycle}

Forces not applied for the whole gait cycle, but tuned for specific (chosen) parts (e.g. subtasks [56], subphases of gait 58], or gait split in bins 43], 59], 60])
Tunable param.

\begin{tabular}{|c|c|}
\hline $\begin{array}{c}\text { Amount of } \\
\text { assistance }\end{array}$ & $\begin{array}{c}\text { Manual tuning } \\
\text { Mostly virtual } \\
\text { Therapists adjusts } \\
\text { tunable parame- } \\
\text { spring stiffness, also }\end{array}$ \\
ters [41], [51], [56]
\end{tabular}

called 'guidance force' [41], [53], [56]

\begin{tabular}{|c|}
\hline Reference \\
trajectory \\
E.g. range of motion \\
61], amplitude or \\
timing of specific gait \\
events/subtasks 56]
\end{tabular}

\section{Automatic tuning}

Tunable parameters are adjusted automatically based on users' performance 43], [58]-60], 62], 63]

\section{Formulation}

\section{Joint space}

Reference trajectories in joint space (e.g.

hip and knee) [41], [50], [56], [57]

\section{End-point space}

Reference trajectories defined with respect

to an anatomical

landmark (e.g. lateral malleolous)

[43], [53], 62]

\begin{tabular}{|c|}
\hline Variability \\
Width of deadband \\
or allowed temporal \\
deviations [50], 53]
\end{tabular}

Combination

joint and endpoint space 60]

reference trajectory adjusted based on users' timing [49]-52]

\section{Combination spa- tial and temporal variability 50]}

Figure 1.4: Overview of several variations that were used for impedance controllers in previous studies. The variations from different columns can be combined. 
such as the Lokomat and ALEX [50], [53]-55].

Another disadvantage of many impedance-based controllers is that the same assistance is applied for the whole gait cycle ('Assistance timing' in Figure 1.4) [41], 51]. This often results in more robotic gait assistance than needed, as most users do not require assistance for the whole gait cycle 52 . Other variations of impedance-based controllers were developed that modulate the assistance over the complete gait cycle to apply assistance only when it is needed [43], [56], [58]-60]. In [43], 59], 60, the gait cycle was split into several bins and assistance was adjusted for each of these bins. However, these bins are not related to functional aspects of gait. This makes it difficult to interpret the resulting assistance levels. Also, it is questionable whether users need assistance for each instance of the gait cycle, or only specific functional aspects of gait. Subtask-based assistance [56], 62 is related to specific functional aspects of gait and common impairments after stroke and SCI (see Section 1.1.2 for more details).

Various parameters can be tuned for impedance-based controllers ('Tunable param.' in Figure 1.4). The tuning process is often focused on the amount of assistance which has a crucial influence on active participation of the user [64]. In most cases, the amount of assistance is defined by the stiffness of a virtual spring (also called 'guidance force' for Lokomat) [41], 51], 56]. The larger the virtual spring stiffness, the higher the applied forces/torques for a specific deviation from the reference trajectory.

The tunable parameters, especially the amount of assistance, have been tuned in two ways in previous studies: manually or automatically ('Assistance tuning' in Figure 1.4. Disadvantages of manual tuning are that the assistance is not continuously adjusted based on patients' performance, and that tuning results depend on subjective decisions of therapists. Automatically-tuned controllers have been developed to overcome these disadvantages and tune the assistance based on users' performance [43], [59], [60], 62], 63], 65]. The amount of assistance (i.e. virtual spring stiffness) for these controllers is adjusted based on deviations from reference trajectories. So far, these controllers adjust the assistance for the whole gait cycle (e.g. split into 30 bins with possibly different assistance levels) [43], [59], 63], each subphase of gait [58] or only assist foot clearance [62]. The control algorithms do not take into account multiple subtasks of gait and related impairments of the users which is further described in Section 1.1.2

In literature, the term 'assist-as-needed' (AAN, also called 'assistance-asneeded') is often used for some of the previously described variations of impedancebased controllers, however, AAN is not clearly defined yet. A broad definition of AAN is that the robotic gait trainer applies the minimal assistance that is needed to perform a specific training task [58, 66]-70]. More specific definitions include that AAN controllers 1. should promote active participation, by systematically decreasing assistance and not allowing for slacking [51], [55, 662, [69, 2. allow for modification of the amount of assistance [41], [51], [56], 3. only assist impaired parts of the gait cycle [51], 52], 62], 4. allow for free movement for unassisted parts of the gait cycle [52], [71] and 5. adjust assistance (in real-time) based on users' performance/capabilities [60], 69], 71]- 74]. Sometimes, 'simple' impedance controllers are called AAN controllers. However, based on above definitions and 
the fact that simple impedance controllers can result in slacking, they should not be called AAN controllers. More advanced controllers, that allow for more spatial and temporal variability, and continuously adjust assistance based on users' performance, match above definitions of AAN better (e.g. [43], 60]).

\section{Subtask-based assistance}

Subtask-based assistance has been implemented in the LOPES II gait trainer and takes into account specific subtasks that are crucial for walking [52, [56]. A subtask is defined as the control of joint movements during a specific part of the gait cycle to complete a specific task. Previous studies suggest that gait can be subdivided into specific functions or tasks that need to be fulfilled to be able to walk. Four 'locomotor functions' (propulsion, stance stability, shock absorption, energy conservation) 75] and five 'prerequisites of gait' (stance stability, swing phase clearance, foot prepositioning, adequate step length, energy conservation) 76] were defined in previous work. These functions/prerequisites, as well as feedback from therapists, were used to define the following subtasks for gait training in LOPES II: weight shift, lateral foot placement, step length, prepositioning, stability during stance and foot clearance (Table 1.1] 52].

Subtask-based assistance could have some advantages compared to previously developed controllers. The subtask-based assistance tackles specific parts of the gait cycle that are often impaired after stroke/SCI (Table 1.1), while allowing for free movements for unimpaired (and thus unassisted) parts of the gait cycle. Also, subtask-based assistance resembles more the way that therapists assist a patient: assessing which subtasks are affected and applying assistance for these specific subtasks only 77]. Focusing on these subtasks also makes manual tuning of the assistance easier for therapists [52]. In addition, assistance can be adjusted to allow for compensatory movements which can play an important role in recovery of gait 78 .

Currently, subtask-based assistance in LOPES II is tuned by the therapist [56] which might have some disadvantages compared to previously described automatically-tuned control algorithms. First, although only specific subtasks are taken into account, the number of parameters that can be tuned is rather large. Subtasks (Table 1.1) can be tuned separately for each leg, and for each subtask the assistance and reference trajectory can be adjusted. Tuning each subtask separately can be difficult and time-intensive for the therapist. Also, assistance is probably not continuously adjusted based on performance within a session. This can result in slacking which reduces active participation and gait variability. Second, the applied assistance depends on subjective decisions. Despite that some feedback is provided to the therapist in a user interface (e.g. maximal knee flexion during swing), therapists can choose to base their decisions on (subjective) visual assessments of the gait pattern.

To sum up, subtask-based assistance could be beneficial compared to previously developed controllers. However, further improvements and a better understanding of the effect of the support is needed to improve subtask-based assistance. Automatically tuning assistance for each subtask based on subtask performance could 
have several advantages. Besides, to get more insight into assistance tuning, this automatic tuning should be compared to manual tuning (by the therapist). Also, it is not known yet whether assistance for specific subtasks in people with neurological disorders leads to only changes for the assisted subtask or an overall change of the gait pattern (i.e. affecting other subtasks or spatiotemporal parameters).

Table 1.1: Overview of subtasks that are assisted with the LOPES II gait trainer and common impairments for these subtasks after stroke and SCI. Unless stated otherwise, subtask performances are compared to unimpaired individuals (e.g. increased/decreased compared to unimpaired individuals). It should be considered that the exact impairments in a specific person with stroke/SCI largely depend on the severity and location of the stroke and SCI. Either one of these impairments or a combination of multiple impairments can be found in most people with stroke/SCI who have problems during walking.

\begin{tabular}{l} 
Subtask \\
\hline $\begin{array}{l}\text { Weight shift (lateral } \\
\text { pelvis displacement) }\end{array}$ \\
Lateral foot place- \\
ment/circumduction
\end{tabular}

(hip abduction during swing phase)

Step length (can be divided into leading limb angle (maximal hip flexion) and trailing limb angle (minimal hip flexion))

Prepositioning (knee flexion angle end of swing phase)

$\begin{array}{lr}\text { Stability during } \\ \text { stance (knee } & \text { flexion } \\ \text { angle during } & \text { swing } \\ \text { phase) } & \end{array}$

Foot clearance (maximal knee flexion angle during swing phase)

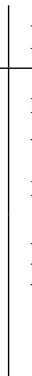

Impairments after stroke
insufficient weight shift to-
wards paretic leg 79$], 80$,
increased weight shift 81
paretic leg: larger step
width and circumduction
83, , 84.

larger step length paretic leg than non-paretic leg 79], 86, paretic leg: decreased hip extension during stance and decreased hip flexion during swing [79], 83], 86- 88

increased knee flexion 88 , 92, 93

Impairments after SCI

insufficient weight shift towards stance leg 82 larger step width 85

decreased step length 85, decreased hip extension during stance [89], 90], decreased hip flexion during swing $89-91$

increased knee flexion 89,94

increased or decreased knee flexion (hyperextension) 79], 86], [88, 93

decreased knee flexion (stiff-knee gait) 79, 83, [87, 88], 93], 95 increased knee flexion [89, 94

decreased knee flexion 90,91 


\subsection{Robot-assisted assessment of sensorimotor impairments and gait}

Robotic devices cannot only be used for gait training, but they can also be valuable tools for assessment of sensorimotor impairments and gait after neurological disorders 96 , 97.

Assessments of sensorimotor impairments and gait are not often performed on a regular basis, although they are important for various reasons [97- 100]. Assessments can be used to track patients' progress and to provide feedback to the therapists, patients and insurance companies. In addition, therapy can be planned, evaluated and adjusted based on assessment outcomes. Even though many therapists acknowledge the importance of assessing their patients' capabilities, sensorimotor assessments are not performed routinely in clinical practice for several reasons (e.g. high costs, time-consuming tasks for the therapists and patients, lack of knowledge and skills, and difficulties understanding outcome measures) 97, 101- 103.

Robot-assisted assessments can be used to overcome some of the factors that prevent the routine use of sensorimotor assessments [97], 100. Measurements from sensors integrated in the devices can lead to objective and sensitive outcome measures. Besides, assessment results depend to a lower degree on therapists' skills and subjective evaluations compared to 'manual assessment' of impairments (i.e. where therapists have to assess impairments by relying on what they feel and/or see). In addition, when assessment is integrated into training devices, no additional setup time is needed and robotic assessment can be time-efficient 97 .

Various body functions can be measured with robotic devices: for example, range of motion (ROM), muscle strength, proprioception, joint torque couplings, joint position sense, joint impedance and walking function 97. With the Lokomat, passive range of motion, lower limb position sense and muscle strength can be measured [97], [104. In this thesis, we are mainly focused on the assessment of joint impedance and walking function as described in the following sections. A comprehensive review of robot-assisted assessment of lower extremity functions can be found in 97 .

\subsubsection{Assessment of joint impedance}

Modulation of joint impedance during gait is crucial to be able to walk in various (challenging) environments 105]. Joint impedance is defined as the mechanical resistance to a perturbation. After stroke and SCI, impairments such as spasticity or hypertonia can lead to changes in the (control of) muscle activity 28, [106, [107]. This can then result in an impaired (modulation of) joint impedance and gait.

Only limited knowledge is available about joint impedance (modulation) during walking. Joint impedance depends on various factors and is assessed by applying a mechanical perturbation with a robotic device to the joint and measuring the response. Previous studies have shown that joint impedance of the ankle, knee and hip in a static situation (e.g. standing or seated) can depend on muscle activity, joint angles and velocities [108]- 115]. Based on these results, variations of joint 
impedance during walking can be expected. For the ankle, a large stiffness during stance phase and a low stiffness during swing phase was found in previous studies 116-118. Measurements of hip and knee joint impedance are needed to get a more complete picture of the modulation of joint impedance during walking and its effect on walking ability. To our knowledge, hip and knee joint impedance have not been determined experimentally during walking yet.

Assessment of joint impedance during walking leads to high requirements for the device used to apply the perturbations. First, the device should be transparent and not affect gait and impedance estimates. Second, the device needs to be able to apply perturbations that can be used to estimate joint impedance using system identification techniques. Current robotic gait trainers are not suited for the assessment of joint impedance during walking as they do not fulfill the abovementioned requirements. Lokomat can be used to measure passive joint stiffness of the hip and knee while the device is moving the leg of the patient [119]. However, for assessments during walking, current robotic gait trainers are not transparent enough and the bandwidth is not high enough to apply the perturbations required for an impedance estimation. Various other robotic devices have been developed to estimate ankle and/or knee joint impedance during walking [120]-[126]. Even though devices for the knee were developed, to our knowledge the time-varying behaviour of knee joint impedance during walking is still unknown. In addition, none of these devices can be used to determine hip joint impedance during walking.

\subsubsection{Assessment of walking function}

Current robotic gait trainers can be used to assess various parameters related to walking function. For example, joint angles and/or interaction forces can usually be extracted from sensors integrated in the devices [33], 56], [127. Also, spatiotemporal parameters are often determined based on measurements from these sensors.

The minimal amount of assistance required by the user can also be a measure to assess walking function and progress during therapy [59]. For example, low assistance levels mean that the user is performing well. High assistance levels are an indication for a diminished performance for the specific assisted aspects of gait. This assessment method can be especially suited for patients who need assistance during walking and therefore might not be able to perform other assessments (i.e. without gait assistance). Automatically-tuned controllers are needed to achieve objective results. Previously developed automatically-tuned algorithms adjusted the assistance for various bins of the gait cycle [43], [59], 60]. These bins were not related to specific functional aspects of gait. Therefore, the outcome measures can be difficult to understand by therapists and patients. Subtask-based assistance (Section 1.1.2 would be easier to interpret as the assisted subtasks are related to specific joints and intervals of the gait cycle. Quantifying these subtasks and monitoring progress could give more insight into patients' progress and the effect of RAGT. This shows again the importance of developing automatically-tuned subtask-based controllers. 


\subsection{Thesis objectives and outline}

Results from previous studies suggest that the effect of RAGT can be improved by personalizing assistance and tailoring the assistance based on patients' impairments [15- 17]. The goal of this thesis is to take a next step towards personalized robot-assisted gait training. To address this goal, we defined two sub-goals:

1. Develop assessment tools to quantify walking function and underlying impairments affecting walking function.

2. Improve subtask-based assistance and optimize assistance tuning based on users' walking abilities.

Modulation of joint impedance is crucial for walking and can be diminished by neurological disorders. The goal of Chapter 2 is to develop and evaluate a device that can be used for the assessment of hip joint impedance during walking, and to determine first estimates of apparent hip joint impedance during walking.

During therapy, walking speed and PBWS are often changed when patients improve their walking ability. This should be taken into account when the applied assistance is used to quantify walking function. In Chapter 3 , we describe an algorithm that automatically adjusts the amount of subtask-based assistance depending on users' performance, and we evaluate whether this algorithm can be used as an assessment tool for walking function when PBWS and walking speed vary.

In clinical practice, robotic assistance is often tuned by the therapist (manuallytuned, MT). This tuning can be difficult and time-consuming, especially when many parameters can be tuned (simultaneously). The automatically-tuned (AT) assistance described in Chapter 3 could possibly lead to faster and more objective tuning of the assistance, however, it has never been compared to MT assistance. In Chapter 4 , differences and similarities between AT and MT assistance in people with chronic stroke and SCI are described, focusing on participants' preferences, the time to tune the assistance, amount of assistance and errors compared to reference trajectories.

Assisting one specific subtask might also influence other subtasks of walking or spatiotemporal gait parameters. Chapter 5 aims to give more insight into the effect of separately tuning impaired subtasks of walking in mildly impaired chronic stroke survivors. The effects on the assisted subtasks, other subtasks of walking and spatiotemporal gait parameters are described.

Chapter 6 contains a general discussion of the results from the previous chapters. Also, limitations of this thesis and future perspectives about (personalized) robot-assisted gait training are given.

\section{References}

[1] W. Pirker and R. Katzenschlager, "Gait disorders in adults and the elderly: A clinical guide", Wiener Klinische Wochenschrift, vol. 129, no. 3-4, pp. 8195, 2017, ISSN: 16137671. DOI: 10.1007/s00508-016-1096-4. 
[2] A. H. Snijders, B. P. van de Warrenburg, N. Giladi, and B. R. Bloem, "Neurological gait disorders in elderly people: clinical approach and classification", Lancet Neurology, vol. 6, no. 1, pp. 63-74, 2007, ISSN: 14744422. DOI: $10.1016 / \mathrm{S} 1474-4422(06) 70678-0$.

[3] H. Stolze, S. Klebe, C. Baecker, C. Zechlin, L. Friege, S. Pohle, and G. Deuschl, "Prevalence of Gait disorders in hospitalized neurological patients", Movement Disorders, vol. 20, no. 1, pp. 89-94, 2005, ISSN: 08853185. DOI: $10.1002 / \mathrm{mds} .20266$.

[4] J. M. Baker, "Gait Disorders", American Journal of Medicine, vol. 131, no. 6, pp. 602-607, 2018, ISSN: 15557162. DOI: $10.1016 / \mathrm{j}$. amjmed. 2017 . 11.051 .

[5] Volksgezondheid en Zorg, Beroerte, Cijfers en Context, Huidige situatie. [Online]. Available: https : / / www . volksgezondheidenzorg . info / onderwerp / beroerte / cijfers - context / huidige - situatie \% 7B \% 5C \# \%7Dnode - prevalentie - en - nieuwe - gevallen - beroerte huisartsenpraktijk (visited on 04/08/2020).

[6] M. Katan and A. Luft, "Global Burden of Stroke", Seminars in Neurology, vol. 38 , no. 2 , pp. 208-211, 2018, ISSN: 10989021 . DOI: $10.1055 / \mathrm{s}-0038-$ 1649503 .

[7] D. Alway and J. W. Cole, Stroke Essentials for Primary Care: A Practical Guide, D. Alway and J. W. Cole, Eds. NewYork: Humana Press, 2009, IsBN: 978-1-934115-01-5. DOI: 10.1007/978-1-59745-433-9.

[8] S. Papa, E. Mauri, F. Rossi, G. Perale, and P. Veglianese, Introduction to spinal cord injury as clinical pathology. Elsevier Ltd, 2020, pp. 1-12, ISBN: 9780081028070. DOI: 10.1016/b978-0-08-102807-0.00001-6.

[9] M. W. Post, C. F. Nooijen, K. Postma, J. Dekkers, F. Penninx, R. J. Van Den Berg-Emons, and H. J. Stam, "People with Spinal Cord Injury in the Netherlands", American Journal of Physical Medicine and Rehabilitation, vol. 96, no. 2, S93-S95, 2017, ISSN: 15377385. DOI: 10.1097 / PHM . 0000000000000619 .

[10] A. De Luca, H. Vernetti, C. Capra, I. Pisu, C. Cassiano, L. Barone, F. Gaito, F. Danese, G. Antonio Checchia, C. Lentino, P. Giannoni, and M. Casadio, "Recovery and compensation after robotic assisted gait training in chronic stroke survivors", Disability and Rehabilitation: Assistive Technology, pp. 1-13, 2018, ISSN: 17483115. DOI: 10.1080/17483107 . 2018 . 1466926 .

[11] S. E. Lord, K. McPherson, H. K. McNaughton, L. Rochester, and M. Weatherall, "Community Ambulation after Stroke: How Important and Obtainable Is It and What Measures Appear Predictive?", Archives of Physical Medicine and Rehabilitation, vol. 85, no. 2, pp. 234-239, 2004, ISSN: 00039993. DOI: $10.1016 /$ j . apmr .2003.05.002 
[12] P. L. Ditunno, M. Patrick, M. Stineman, and J. F. Ditunno, "Who wants to walk? Preferences for recovery after SCI: A longitudinal and cross-sectional study", Spinal Cord, vol. 46, no. 7, pp. 500-506, 2008, ISSN: 13624393. DOI: $10.1038 / \mathrm{sj}$.sc.3102172.

[13] J. Mehrholz, S. Thomas, C. Werner, J. Kugler, M. Pohl, and B. Elsner, "Electromechanical-assisted training for walking after stroke (Review)", Cochrane Database of Systematic Reviews, no. 5, 2017. Dor: 10 . 1002/ 14651858.CD006185.pub4.

[14] J. E. Cho, J. S. Yoo, K. E. Kim, S. T. Cho, W. S. Jang, K. H. Cho, and W. H. Lee, "Systematic Review of Appropriate Robotic Intervention for Gait Function in Subacute Stroke Patients", BioMed Research International, vol. 2018, 2018, ISSN: 23146141. DOI: 10.1155/2018/4085298.

[15] S. F. Atashzar, M. Shahbazi, and R. V. Patel, "Haptics-enabled Interactive NeuroRehabilitation Mechatronics: Classification, Functionality, Challenges and Ongoing Research", Mechatronics, vol. 57, pp. 1-19, 2019, ISSN: 09574158. DOI: $10.1016 /$ j.mechatronics.2018.03.002

[16] R. Colombo and V. Sanguineti, Assistive controllers and modalities for robot-aided neurorehabilitation. Elsevier Ltd., 2018, pp. 63-74, ISBN: 9780128119952. DOI: 10.1016/b978-0-12-811995-2.00005-9.

[17] R. Gassert and V. Dietz, "Rehabilitation robots for the treatment of sensorimotor deficits: A neurophysiological perspective", Journal of NeuroEngineering and Rehabilitation, vol. 15, no. 1, pp. 1-15, 2018, ISSN: 17430003. DOI: $10.1186 / \mathrm{s} 12984-018-0383-\mathrm{x}$.

[18] M. Maier, B. R. Ballester, and P. F. Verschure, "Principles of Neurorehabilitation After Stroke Based on Motor Learning and Brain Plasticity Mechanisms", Frontiers in Systems Neuroscience, vol. 13, no. 74, 2019, ISSN: 16625137. DOI: 10.3389/fnsys.2019.00074.

[19] I. J. Hubbard, M. W. Parsons, C. Neilson, and L. M. Carey, "Task-specific training: evidence for and translation to clinical practice", Occupational therapy international, vol. 16, no. 3-4, pp. 175-189, 2009, ISSN: 1557-0703. DOI: $10.1002 /$ oti.

[20] N. A. Bayona, J. Bitensky, K. Salter, and R. Teasell, "The role of taskspecific training in rehabilitation therapies", Topics in Stroke Rehabilitation, vol. 12, no. 3, pp. 58-65, 2005, ISSN: 10749357. DOI: 10.1310/BQM56YGB-MVJ5-WVCR.

[21] M. D. Kubasak, D. L. Jindrich, H. Zhong, A. Takeoka, K. C. McFarland, C. Muñoz-Quiles, R. R. Roy, V. R. Edgerton, A. Ramón-Cueto, and P. E. Phelps, "OEG implantation and step training enhance hindlimb-stepping ability in adult spinal transected rats", Brain, vol. 131, no. 1, pp. 264-276, 2008, ISSN: 14602156. DOI: 10.1093/brain/awm267. 
[22] K. J. Sullivan, D. A. Brown, T. Klassen, S. Mulroy, T. Ge, S. P. Azen, and C. J. Winstein, "Effects of Task-Specific Locomotor and Strength Training in Adults Who Were Ambulatory After Stroke: Results of the STEPS Randomized Clinical Trial", Physical Therapy, vol. 87, no. 12, pp. 1580-1602, 2007, ISSN: 0031-9023. DOI: 10.2522/ptj . 20060310.

[23] D. S. Magnuson, R. R. Smith, E. H. Brown, G. Enzmann, C. Angeli, P. M. Quesada, and D. Burke, "Swimming as a model of task-specific locomotor retraining after spinal cord injury in the rat", Neurorehabilitation and Neural Repair, vol. 23, no. 6, pp. 535-545, 2009, ISSN: 15459683 . DOI: $10.1177 /$ 1545968308331147.

[24] M. Iqbal, A. Arsh, S. M. Hammad, I. U. Haq, and H. Darain, "Comparison of dual task specific training and conventional physical therapy in ambulation of hemiplegic stroke patients: A randomized controlled trial", JPMA. The Journal of the Pakistan Medical Association, vol. 70, no. 1, pp. 7-10, 2020, ISSN: 00309982. DOI: 10.5455/JPMA.10443.

[25] T. G. Hornby, D. S. Reisman, I. G. Ward, P. L. Scheets, A. Miller, D. Haddad, E. J. Fox, N. E. Fritz, K. Hawkins, C. E. Henderson, K. L. Hendron, C. L. Holleran, J. E. Lynskey, and A. Walter, Clinical Practice Guideline to Improve Locomotor Function Following Chronic Stroke, Incomplete Spinal Cord Injury, and Brain Injury, 1. 2020, vol. 44, pp. 49-100, ISBN: 0000000000000. DOI: 10.1097/NPT.0000000000000303.

[26] G. Kwakkel, R. Van Peppen, R. C. Wagenaar, S. W. Dauphinee, C. Richards, A. Ashburn, K. Miller, N. Lincoln, C. Partridge, I. Wellwood, and P. Langhorne, "Effects of augmented exercise therapy time after stroke: A meta-analysis", Stroke, vol. 35, no. 11, pp. 2529-2536, 2004, ISSN: 00392499. DOI: $10.1161 / 01$. STR.0000143153.76460.7d.

[27] B. French, T. Lh, J. Coupe, M. Ne, L. Connell, J. Harrison, S. Cj, and S. Tishkovskaya, "Repetitive task training for improving functional ability after stroke (Review)", Cochrane Database of Systematic Reviews, no. 11, 2016. DOI: 10 . 1002/14651858 . CD006073 . pub3 . www . cochranelibrary . com.

[28] V. Dietz and T. Sinkjaer, "Spastic movement disorder: impaired reflex function and altered muscle mechanics", Lancet Neurology, vol. 6, no. 8, pp. 725733, Aug. 2007, ISSN: 14744422. DOI: 10.1016/S1474-4422(07)70193-X.

[29] M. R. Spiess, F. Steenbrink, and A. Esquenazi, "Getting the Best Out of Advanced Rehabilitation Technology for the Lower Limbs: Minding Motor Learning Principles", Innovations Influencing Physical Medicine and Rehabilitation, vol. 10, no. 9, S165-S173, 2018, ISSN: 19341482. DOI: 10.1016/ j.pmrj.2018.06.007

[30] P. K. Shah, Y. Gerasimenko, A. Shyu, I. Lavrov, H. Zhong, R. R. Roy, and V. R. Edgerton, "Variability in step training enhances locomotor recovery after a spinal cord injury", European Journal of Neuroscience, vol. 36, no. 1, pp. 2054-2062, 2012, ISSN: 0953816X. DOI: $10.1111 / \mathrm{j} .1460-9568.2012$. $08106 . x$. 
[31] R. A. Scheidt, D. J. Reinkensmeyer, M. A. Conditt, W. Zev Rymer, and F. A. Mussa-Ivaldi, "Persistence of motor adaptation during constrained, multi-joint, arm movements", Journal of Neurophysiology, vol. 84, no. 2, pp. 853-862, 2000, ISSN: 00223077. DOI: 10.1152/jn.2000.84.2.853.

[32] M. Lotze, C. Braun, N. Birbaumer, S. Anders, and L. G. Cohen, "Motor learning elicited by voluntary drive", Brain, vol. 126, no. 4, pp. 866-872, 2003, ISSN: 00068950. DOI: 10.1093/brain/awg079.

[33] G. Colombo, M. Joerg, R. Schreier, and V. Dietz, "Treadmill training of paraplegic patients using a robotic orthosis", Journal of Rehabilitation Research \& Development, vol. 37, no. 6, pp. 693-700, 2000, ISSN: 00315125. DOI: $10.2466 / \mathrm{pms} .1991 .73 .1 .146$.

[34] M. F. Bruni, C. Melegari, M. C. De Cola, A. Bramanti, P. Bramanti, and R. S. Calabrò, "What does best evidence tell us about robotic gait rehabilitation in stroke patients: A systematic review and meta-analysis", Journal of Clinical Neuroscience, vol. 48, pp. 11-17, 2018, ISSN: 15322653. DOI: 10.1016/j.jocn.2017.10.048.

[35] J. Mehrholz, L. A. Harvey, S. Thomas, and B. Elsner, "Is body-weight supported treadmill training or robotic-assisted gait training superior to overground gait training and other forms of physiotherapy in people with spinal cord injury? A systematic review", Spinal Cord, vol. 56, no. 4, p. 412, 2018, ISSN: 14765624. DOI: 10.1038/s41393-017-0059-6.

[36] C.-Y. Hsu, Y.-H. Cheng, C.-H. Lai, and Y.-N. Lin, "Clinical non-superiority of technology-assisted gait training with body weight support in patients with subacute stroke: A meta-analysis.", Annals of physical and rehabilitation medicine, 2019, ISSN: 1877-0665 (Electronic). DOI: 10.1016/j.rehab. 2019.09.009.

[37] S. C. Hayes, C. R. James Wilcox, H. S. Forbes White, and N. Vanicek, "The effects of robot assisted gait training on temporal-spatial characteristics of people with spinal cord injuries: A systematic review", The Journal of Spinal Cord Medicine, vol. 0, no. 0, pp. 1-15, 2018, ISSN: 1079-0268. DOI: 10.1080/10790268.2018.1426236.

[38] M. Holden, K. Gill, M. Magliozzi, J. Nathan, and L. Piehl-Baker, "Clinical gait assessment in the neurologically impaired. Reliability and meaningfulness", Physical Therapy, vol. 64, no. 1, pp. 35-40, 1984, ISSN: 00319023. DOI: $10.1093 / \mathrm{ptj} / 64.1 .35$.

[39] L. Marchal-Crespo and R. Riener, Robot-assisted gait training. Elsevier Ltd., 2018, pp. 227-240, ISBN: 9780128119952. DOI: 10 . 1016 /b978-012-811995-2.00016-3.

[40] B. Koopman, E. H. F. van Asseldonk, and H. van der Kooij, "Speeddependent reference joint trajectory generation for robotic gait support", Journal of Biomechanics, vol. 47, no. 6, pp. 1447-1458, 2014, ISSN: 18732380. DOI: $10.1016 / \mathrm{j} \cdot \mathrm{jbiomech.2014.01.037.}$ 
[41] R. Riener, L. Lünenburger, I. C. Maier, G. Colombo, and V. Dietz, "Locomotor training in subjects with sensori-motor deficits: An overview of the robotic gait orthosis Lokomat", Journal of Healthcare Engineering, vol. 1, no. 2, pp. 197-216, 2010, ISSN: 20402309. DOI: 10.1260/2040-2295.1.2. 197.

[42] H. Vallery, E. H. F. van Asseldonk, M. Buss, and H. van der Kooij, "Reference trajectory generation for rehabilitation robots: Complementary limb motion estimation", IEEE Transactions on Neural Systems and Rehabilitation Engineering, vol. 17, no. 1, pp. 23-30, 2009, ISSN: 15344320. DOI: 10.1109/TNSRE. 2008.2008278.

[43] J. L. Emken, S. J. Harkema, J. A. Beres-Jones, C. K. Ferreira, and D. J. Reinkensmeyer, "Feasibility of manual teach-and-replay and continuous impedance shaping for robotic locomotor training following spinal cord injury", IEEE Transactions on Biomedical Engineering, vol. 55, no. 1, pp. 322-334, 2008, ISSN: 00189294. DOI: 10.1109/TBME.2007.910683

[44] A. R. Wu, F. Dzeladini, T. J. H. Brug, F. Tamburella, N. L. Tagliamonte, E. H. F. van Asseldonk, H. van der Kooij, and A. J. Ijspeert, "An adaptive neuromuscular controller for assistive lower-limb exoskeletons: A preliminary study on subjects with spinal cord injury", Frontiers in Neurorobotics, vol. 11, no. JUN, pp. 1-14, 2017, ISSN: 16625218. DOI: $10.3389 /$ fnbot . 2017.00030.

[45] G. Durandau, D. Farina, G. Asín-Prieto, I. Dimbwadyo-Terrer, S. LermaLara, J. L. Pons, J. C. Moreno, and M. Sartori, "Voluntary control of wearable robotic exoskeletons by patients with paresis via neuromechanical modeling", Journal of NeuroEngineering and Rehabilitation, vol. 16, no. 1, pp. 1-18, 2019, ISSN: 17430003. DOI: 10.1186/s12984-019-0559-z.

[46] S. A. Murray, K. H. Ha, C. Hartigan, and M. Goldfarb, "An assistive control approach for a lower-limb exoskeleton to facilitate recovery of walking following stroke", IEEE Transactions on Neural Systems and Rehabilitation Engineering, vol. 23, no. 3, pp. 441-449, 2015, ISSN: 15344320. DOI: 10.1109/TNSRE.2014.2346193

[47] D. J. Reinkensmeyer, O. M. Akoner, D. P. Ferris, and K. E. Gordon, "Slacking by the human motor system: Computational models and implic implications for robotic orthoses", Proceedings of the 31st Annual International Conference of the IEEE Engineering in Medicine and Biology Society: Engineering the Future of Biomedicine, EMBC 2009, pp. 2129-2132, 2009. DOI: $10.1109 /$ IEMBS. 2009.5333978

[48] R. Riener, L. Lünenburger, S. Jezernik, M. Anderschitz, G. Colombo, and V. Dietz, "Patient-cooperative strategies for robot-aided treadmill training: First experimental results", IEEE Transactions on Neural Systems and Rehabilitation Engineering, vol. 13, no. 3, pp. 380-394, 2005, ISSN: 15344320. DOI: $10.1109 /$ TNSRE. 2005.848628 
[49] D. Aoyagi, W. Ichinose, S. Harkema, D. Reinkensmeyer, and J. Bobrow, "A Robot and Control Algorithm That Can Synchronously Assist in Naturalistic Motion During Body-Weight-Supported Gait Training Following Neurologic Injury", IEEE Transactions on Neural Systems and Rehabilitation Engineering, vol. 15, no. 3, pp. 387-400, 2007, ISSN: 1534-4320. DOI: 10.1109/TNSRE. 2007.903922.

[50] A. Duschau-Wicke, J. Von Zitzewitz, A. Caprez, L. Lünenburger, and R. Riener, "Path control: A method for patient-cooperative robot-aided gait rehabilitation", IEEE Transactions on Neural Systems and Rehabilitation Engineering, vol. 18, no. 1, pp. 38-48, 2010, ISSN: 15344320. DOI:10.1109/ TNSRE. 2009.2033061.

[51] B. M. Fleerkotte, B. Koopman, J. H. Buurke, E. H. F. van Asseldonk, H. van der Kooij, and J. S. Rietman, "The effect of impedance-controlled robotic gait training on walking ability and quality in individuals with chronic incomplete spinal cord injury: An explorative study", Journal of NeuroEngineering and Rehabilitation, vol. 11, no. 26, 2014, ISSN: 17430003. DOI: 10.1186/1743-0003-11-26.

[52] J. Meuleman, "Design of a Robot-Assisted Gait Trainer: LOPES II", PhD thesis, University of Twente, 2015, pp. 1-300, ISBN: 9789036539654. DOI: $10.3990 / 1.9789036539654$.

[53] S. K. Banala, S. H. Kim, S. K. Agrawal, and J. P. Scholz, "Robot assisted gait training with active leg exoskeleton (ALEX)", Proceedings of the 2nd Biennial IEEE/RAS-EMBS International Conference on Biomedical Robotics and Biomechatronics, BioRob 2008, vol. 17, no. 1, pp. 653-658, 2008. DOI: 10.1109/BIOROB. 2008.4762885.

[54] S. Srivastava, P. C. Kao, S. H. Kim, P. Stegall, D. Zanotto, J. S. Higginson, S. K. Agrawal, and J. P. Scholz, "Assist-as-Needed Robot-Aided Gait Training Improves Walking Function in Individuals Following Stroke", IEEE Transactions on Neural Systems and Rehabilitation Engineering, vol. 23, no. 6, pp. 956-963, 2015, ISSN: 15344320. DOI: 10.1109/TNSRE. 2014 . 2360822.

[55] P. Stegall, D. Zanotto, and S. K. Agrawal, "Variable damping force tunnel for gait training using ALEX III", Physiology and Behavior, vol. 2, no. 3, pp. 1495-1501, 2017, ISSN: 1873507X. DOI: 10.1016/j.physbeh.2017.03. 040 .

[56] J. Meuleman, E. H. van Asseldonk, G. van Oort, H. Rietman, and H. van der Kooij, "LOPES II - Design and Evaluation of an Admittance Controlled Gait Training Robot with Shadow-Leg Approach", IEEE Transactions on Neural Systems and Rehabilitation Engineering, vol. 24, no. 3, pp. 352-363, 2016, ISSN: 15344320. DOI: 10.1109/TNSRE.2015.2511448. 
[57] J. F. Veneman, R. Kruidhof, E. E. Hekman, R. Ekkelenkamp, E. H. F. van Asseldonk, and H. van der Kooij, "Design and Evaluation of the LOPES Exoskeleton Robot for Interactive Gait Rehabilitation", IEEE Transactions on Neural Systems and Rehabilitation Engineering, vol. 15, no. 3, pp. 379386, 2007, ISSN: 07907915.

[58] G. Chen, J. Ye, Q. Liu, L. Duan, W. Li, Z. Wu, and C. Wang, "Adaptive control strategy for gait rehabilitation robot to assist-when-needed", 2018 IEEE International Conference on Real-Time Computing and Robotics, RCAR 2018, pp. 538-543, 2018. DOI: 10.1109/RCAR.2018.8621706.

[59] S. Maggioni, L. Lünenburger, R. Riener, and A. Melendez-Calderon, "Robot-Aided assessment of walking function based on an adaptive algorithm", IEEE International Conference on Rehabilitation Robotics, pp. 804-809, 2015, ISSN: 19457901. DOI: 10.1109/ICORR.2015.7281301.

[60] S. Maggioni, N. Reinert, L. Lünenburger, and A. Melendez-Calderon, "An Adaptive and Hybrid End-Point/Joint Impedance Controller for Lower Limb Exoskeletons", Frontiers in Robotics and AI, vol. 5, no. 104, 2018, ISSN: 1344-3542. DOI: $10.3389 /$ frobt.2018.00104.

[61] Hocoma, Lokomat User Script, 2018. [Online]. Available: https : / / knowledge . hocoma . com / wp-content / uploads / 2019/03/Lokomat \% 7B\% 5C_\%7DUser\%7B\%5C_\%7DScript\%7B\%5C_\%7DEN\%7B\%5C_\%7D20180322.pdf.

[62] B. Koopman, E. H. F. van Asseldonk, and H. van der Kooij, "Selective control of gait subtasks in robotic gait training: Foot clearance support in stroke survivors with a powered exoskeleton", Journal of NeuroEngineering and Rehabilitation, vol. 10, no. 1, 2013, ISSN: 17430003. DOI: 10.1186/17430003-10-3.

[63] D. Zanotto, P. Stegall, and S. K. Agrawal, "Adaptive assist-as-needed controller to improve gait symmetry in robot-assisted gait training", Proceedings - IEEE International Conference on Robotics and Automation, pp. 724-729, 2014, ISSN: 10504729. DOI: 10.1109/ICRA.2014.6906934

[64] I. J. Park, J.-H. Park, H. Y. Seong, J. S. H. You, S. J. Kim, J. H. Min, H. Y. Ko, and Y.-I. Shin, "Comparative Effects of Different Assistance Force During Robot-Assisted Gait Training on Locomotor Functions in Patients with Subacute Stroke", American Journal of Physical Medicine \& Rehabilitation, vol. 98, no. 1, pp. 58-64, 2018, ISSN: 0894-9115. DOI: $10.1097 / \mathrm{phm} .0000000000001027$.

[65] C. Bayón, "Design, Development and Evaluation of a Robotic Platform for Gait Rehabilitation and Training in Patients with Cerebral Palsy", PhD thesis, 2018. DOI: $10.1016 /$ j.robot.2016.12.015

[66] M. Ferrarin, M. Rabuffetti, E. Geda, S. Sirolli, A. Marzegan, V. Bruno, and K. Sacco, "Influence of the amount of body weight support on lower limb joints' kinematics during treadmill walking at different gait speeds: Reference data on healthy adults to define trajectories for robot assistance", Proceedings of the Institution of Mechanical Engineers, Part H: Journal of 
Engineering in Medicine, vol. 232, no. 6, pp. 619-627, 2018, ISSN: 20413033. DOI: $10.1177 / 0954411918776682$.

[67] A. Duschau-Wicke, A. Caprez, and R. Riener, "Patient-cooperative control increases active participation of individuals with SCI during robot-aided gait training", Journal of NeuroEngineering and Rehabilitation, vol. 7, no. 1, pp. 1-13, 2010, ISSN: 17430003. DOI: 10.1186/1743-0003-7-43

[68] L. Marchal-Crespo and D. J. Reinkensmeyer, "Review of control strategies for robotic movement training after neurologic injury", Journal of NeuroEngineering and Rehabilitation, vol. 6, no. 1, p. 20, 2009, ISSN: 1743-0003. DOI: $10.1186 / 1743-0003-6-20$.

[69] J. L. Emken, J. E. Bobrow, and D. J. Reinkensmeyer, "Robotic movement training as an optimization problem: Designing a controller that assists only as needed", Proceedings of the 2005 IEEE 9th International Conference on Rehabilitation Robotics, vol. 2005, pp. 307-312, 2005. DOI: 10.1109/ICORR. 2005.1501108.

[70] J. L. Emken, R. Benitez, and D. J. Reinkensmeyer, "Human-robot cooperative movement training: Learning a novel sensory motor transformation during walking with robotic assistance-as-needed", Journal of NeuroEngineering and Rehabilitation, vol. 4, pp. 1-14, 2007, ISSN: 17430003. DOI: 10.1186/1743-0003-4-8.

[71] H. J. Asl and J. Yoon, "Stable assist-as-needed controller design for a planar cable-driven robotic system", International Journal of Control, Automation and Systems, vol. 15, no. 6, pp. 2871-2882, 2017, ISSN: 20054092. DOI: 10. 1007/s12555-016-0492-x

[72] J. Cao, S. Q. Xie, R. Das, and G. L. Zhu, "Control strategies for effective robot assisted gait rehabilitation: the state of art and future prospects.", Medical engineering \& physics, vol. 36, no. 12, pp. 1555-66, 2014, ISSN: 1873-4030. DOI: 10.1016/j.medengphy.2014.08.005.

[73] S. Y. A. Mounis, N. Z. Azlan, and S. Fatai, "Progress based assist-asneeded control strategy for upper-limb rehabilitation", Proceedings - 2017 IEEE Conference on Systems, Process and Control, ICSPC 2017, pp. 6570, 2017. DOI: 10.1109/SPC.2017.8313023

[74] R. Riener, "Rehabilitation Robotics", Foundations and Trends in Robotics, vol. 3, no. 1-2, pp. 1-137, 2012, ISSN: 1935-8253. DOI: 10.1561/2300000028.

[75] J. Perry and J. M. Burnfield, Gait Analysis, Normal and Pathological Function. 1st ed. Thorofare, New Jersey: SLACK Incorporated, 1992, pp. 1-576, ISBN: 9781556421921. DOI: 10.2106/00004623-199303000-00027.

[76] J. R. Gage, Gait analysis: An essential tool in the treatment of cerebral palsy, 288. Lippincott-Raven Publishers, 1993, vol. 288, pp. 126-134. DOI: 10.1097/00003086-199303000-00016. 
[77] D. J. Reinkensmeyer, J. A. Galvez, L. Marchai, E. T. Wolbrecht, and J. E. Bobrow, "Some key problems for robot-assisted movement therapy research: A perspective from the University of California at Irvine", 2007 IEEE 10th International Conference on Rehabilitation Robotics, ICORR'0\%, vol. 00, no. c, pp. 1009-1015, 2007. DOI: 10.1109/ICORR.2007.4428547.

[78] J. H. Buurke, A. V. Nene, G. Kwakkel, V. Erren-Wolters, M. J. Ijzerman, and H. J. Hermens, "Recovery of gait after stroke: What changes?", Neurorehabilitation and Neural Repair, vol. 22, no. 6, pp. 676-683, 2008, ISSN: 15459683. DOI: $10.1177 / 1545968308317972$.

[79] B. Balaban and F. Tok, "Gait Disturbances in Patients With Stroke", PM $R$, vol. 6 , no. 7 , pp. 635-642, 2014, ISSN: 19341482 . DOI: $10.1016 / \mathrm{j} \cdot \mathrm{pmrj}$. 2013.12.017.

[80] H. Y. Hsiao, V. L. Gray, R. A. Creath, S. A. Binder-Macleod, and M. W. Rogers, "Control of lateral weight transfer is associated with walking speed in individuals post-stroke", Journal of Biomechanics, vol. 60, pp. 72-78, 2017, ISSN: 18732380. DOI: 10.1016/j.jbiomech.2017.06.021.

[81] K. J. Dodd and M. E. Morris, "Lateral pelvic displacement during gait: Abnormalities after stroke and changes during the first month of rehabilitation", Archives of Physical Medicine and Rehabilitation, vol. 84, no. 8, pp. 1200-1205, 2003, ISSN: 00039993. DOI: 10.1016 / S0003-9993(03 ) 00142-4.

[82] M. Wu, J. Kim, and F. Wei, "Facilitating Weight Shifting during Treadmill Training Improves Walking Function in Humans with Spinal Cord Injury: A Randomized Controlled Pilot Study", American Journal of Physical Medicine and Rehabilitation, vol. 97, no. 8, pp. 585-592, 2018, ISSN: 15377385. DOI: 10.1097/PHM.0000000000000927.

[83] G. Chen, C. Patten, D. H. Kothari, and F. E. Zajac, "Gait differences between individuals with post-stroke hemiparesis and non-disabled controls at matched speeds.", Gait \& posture, vol. 22, no. 1, pp. 51-6, Aug. 2005, ISSN: 0966-6362. DOI: $10.1016 / \mathrm{j}$.gaitpost.2004.06.009.

[84] V. A. Stanhope, B. A. Knarr, D. S. Reisman, and S. Jill, "Frontal plane compensatory strategies associated with self- selected walking speed in individuals post-stroke", vol. 29, no. 5, pp. 518-522, 2015. DOI: $10.1016 / \mathrm{j}$. clinbiomech.2014.03.013.Frontal

[85] A. T. Murphy, S. Kravtsov, M. Sangeux, B. Rawicki, and P. W. New, "Utilizing three dimensional clinical gait analysis to optimize mobility outcomes in incomplete spinal cord damage", Gait and Posture, vol. 74, no. August, pp. 53-59, 2019, ISSN: 18792219. DOI: 10.1016/j.gaitpost.2019.08.001

[86] L. R. Sheffler and J. Chae, "Hemiparetic Gait", Physical Medicine and Rehabilitation Clinics of North America, vol. 26, no. 4, pp. 611-623, 2015, ISSN: 15581381 . DOI: $10.1016 / \mathrm{j} \cdot \mathrm{pmr} .2015 .06 .006$ 
[87] E. Hutin, D. Pradon, F. Barbier, J.-M. Gracies, B. Bussel, and N. Roche, "Lower limb coordination patterns in hemiparetic gait: factors of knee flexion impairment.", Clinical biomechanics (Bristol, Avon), vol. 26, no. 3, pp. 304-11, Mar. 2011, ISSN: 1879-1271. DOI: $10.1016 / \mathrm{j}$. clinbiomech . 2010.10.007.

[88] S. Mulroy, J. A. Gronley, W. Weiss, C. Newsam, and J. Perry, "Use of cluster analysis for gait pattern classification of patients in the early and late recovery phases following stroke", Gait and Posture, vol. 18, no. 1, pp. 114-125, 2003, ISSN: 09666362. DOI: 10.1016/S0966-6362(02)001650 .

[89] A. Domingo, G. S. Sawicki, and D. P. Ferris, "Kinematics and muscle activity of individuals with incomplete spinal cord injury during treadmill stepping with and without manual assistance", Journal of NeuroEngineering and Rehabilitation, vol. 4, pp. 1-14, 2007, ISSN: 17430003. DOI: 10 . 1186/1743-0003-4-32.

[90] A. van der Salm, A. V. Nene, D. J. Maxwell, P. H. Veltink, H. J. Hermens, and M. J. Ijzerman, "Gait impairments in a group of patients with incomplete spinal cord injury and their relevance regarding therapeutic approaches using functional electrical stimulation", Artificial Organs, vol. 29, no. 1, pp. 8-14, 2005, ISSN: 0160564X. DOI: $10.1111 / \mathrm{j} .1525-1594.2004$. $29004 . x$

[91] H. Barbeau, S. Nadeau, and C. Garneau, "Physical Determinants, Emerging Concepts, and Training Approaches in Gait of Individuals with Spinal Cord Injury", Journal of Neurotrauma, vol. 23, no. 3, pp. 571-585, 2006. DOI: $10.1089 /$ neu.2006.23.571.

[92] P. R. Trueblood, J. M. Walker, J. Perry, and J. K. Gronley, "Pelvic exercise and gait in hemiplegia", Physical Therapy, vol. 69, no. 1, pp. 18-26, 1989, ISSN: 00319023. DOI: $10.1093 / \mathrm{ptj} / 69.1 .18$.

[93] I. Kramers De Quervain, S. Simon, S. Leurgans, W. Pease, and D. McAllister, "Gait pattern in the early recovery period after stroke", Journal of Bone and Joint Surgery - Series A, vol. 78, no. 10, pp. 1506-1514, 1996.

[94] H. Barbeau, M. Ladouceq, K. E. Norman, A. Pépin, and A. Leroux, "Walking After Spinal Cord Injury: Evaluation, Treatment and Functional Recovery", Archiv, vol. 80, pp. 225-235, 1999. DOI: https://doi .org/10. 1016/S0003-9993(99)90126-0.

[95] I. Campanini, A. Merlo, and B. Damiano, "A method to differentiate the causes of stiff-knee gait in stroke patients", Gait and Posture, vol. 38, no. 2, pp. 165-169, 2013, ISSN: 09666362. DOI: $10.1016 / \mathrm{j} \cdot$ gaitpost. 2013.05 . 003.

[96] J. Hidler, D. Nichols, M. Pelliccio, and K. Brady, "Advances in the understanding and treatment of stroke impairment using robotic devices", Topics in Stroke Rehabilitation, vol. 12, no. 2, pp. 22-35, 2005, ISSN: 10749357. DOI: 10.1310/RYT5-62N4-CTVX-8JTE. 
[97] S. Maggioni, A. Melendez-Calderon, E. H. F. van Asseldonk, V. KlamrothMarganska, L. Lünenburger, R. Riener, and H. van der Kooij, Robot-aided assessment of lower extremity functions: A review, 2016. DOI: 10.1186/ s12984-016-0180-3.

[98] E. A. Duncan and J. Murray, "The barriers and facilitators to routine outcome measurement by allied health professionals in practice: A systematic review", BMC Health Services Research, vol. 12, no. 96, 2012, ISSN: 14726963. DOI: 10.1186/1472-6963-12-96.

[99] D. U. Jette, J. Halbert, C. Iverson, E. Miceli, and P. Shah, "Use of Standardized Outcome Measures in Physical Therapist Practice: Perceptions and Applications", Physical Therapy, vol. 89, no. 2, pp. 125-135, 2009, ISSN: 0031-9023. DOI: 10.2522/pt j.20080234.

[100] C. Shirota, S. Balasubramanian, and A. Melendez-Calderon, "Technologyaided assessments of sensorimotor function: current use, barriers and future directions in the view of different stakeholders", Journal of NeuroEngineering and Rehabilitation, vol. 16, no. 1, p. 53, 2019, ISSN: 1743-0003. DOI: 10.1186/s12984-019-0519-7.

[101] N. Otterman, J. Veerbeek, S. Schiemanck, P. van der Wees, F. Nollet, and G. Kwakkel, "Selecting relevant and feasible measurement instruments for the revised Dutch clinical practice guideline for physical therapy in patients after stroke", Disability and Rehabilitation, vol. 39, no. 14, pp. 1449-1457, 2017, ISSN: 14645165. DOI: 10.1080/09638288.2016.1196399.

[102] T. N. Al-Muqiren, E. S. Al-Eisa, A. H. Alghadir, and S. Anwer, "Implementation and use of standardized outcome measures by physical therapists in Saudi Arabia: Barriers, facilitators and perceptions", BMC Health Services Research, vol. 17, no. 1, pp. 1-10, 2017, ISSN: 14726963. DOI: $10.1186 /$ s12913-017-2693-2.

[103] T. Braun, A. Rieckmann, F. Weber, and C. Grüneberg, "Current use of measurement instruments by physiotherapists working in Germany: A cross-sectional online survey", BMC Health Services Research, vol. 18, no. 1, pp. 1-16, 2018, ISSN: 14726963. DOI: 10.1186/s12913-018-3563-2.

[104] A. Domingo and T. Lam, "Reliability and validity of using the Lokomat to assess lower limb joint position sense in people with incomplete spinal cord injury", Journal of NeuroEngineering and Rehabilitation, vol. 11, no. 1, pp. 1-10, 2014, ISSN: 17430003. DOI: 10.1186/1743-0003-11-167.

[105] H. Lee, E. J. Rouse, and H. I. Krebs, "Summary of Human Ankle Mechanical Impedance during Walking", IEEE Journal of Translational Engineering in Health and Medicine, vol. 4, no. September, 2016, ISSN: 21682372. DOI: $10.1109 /$ JTEHM. 2016.2601613.

[106] A. Roy, H. I. Krebs, C. T. Bever, L. W. Forrester, R. F. Macko, and N. Hogan, "Measurement of passive ankle stiffness in subjects with chronic hemiparesis using a novel ankle robot", Journal of Neurophysiology, vol. 105, no. 5, pp. 2132-2149, 2011, ISSN: 0022-3077. DOI: 10.1152/ jn.01014.2010 
[107] H. Lee, T. Patterson, J. Ahn, D. Klenk, A. Lo, H. I. Krebs, and N. Hogan, "Static ankle impedance in stroke and multiple sclerosis: A feasibility study", Proceedings of the Annual International Conference of the IEEE Engineering in Medicine and Biology Society, EMBS, pp. 8523-8526, 2011, ISSN: 1557170X. DOI: 10.1109/IEMBS.2011.6092103

[108] B. Koopman, E. H. F. van Asseldonk, and H. van der Kooij, "Estimation of Human Hip and Knee Multi-Joint Dynamics Using the LOPES Gait Trainer", IEEE Transactions on Robotics, vol. 32, no. 4, pp. 920-932, 2016, ISSN: 15523098. DOI: 10.1109/TRO.2016.2572695.

[109] D. Ludvig, M. Plocharski, P. Plocharski, and E. J. Perreault, "Mechanisms contributing to reduced knee stiffness during movement", Experimental Brain Research, vol. 235, no. 10, pp. 1-12, 2017, ISSN: 14321106. DOI: $10.1007 / \mathrm{s} 00221-017-5032-2$.

[110] L. Q. Zhang, G. Nuber, J. Butler, M. Bowen, and W. Z. Rymer, "In vivo human knee joint dynamic properties as functions of muscle contraction and joint position", Journal of Biomechanics, vol. 31, no. 1, pp. 71-76, 1997, ISSN: 00219290. DOI: 10.1016/S0021-9290(97)00106-1.

[111] H.-Y. Huang, A. Arami, I. Farkhatdinov, D. Formica, and E. Burdet, "The influence of posture, applied force and perturbation direction on hip joint viscoelasticity", ArXiv e-prints, pp. 1-7, 2019. arXiv: 1908.07800.

[112] P. L. Weiss, R. E. Kearney, and I. W. Hunter, "Position dependence of ankle joint dynamics-I. Passive mechanics", Journal of Biomechanics, vol. 19, no. 9, pp. 727-735, 1986, ISSN: 00219290. DOI: 10.1016/0021-9290(86) 90196-X.

[113] G. L. Gottlieb and G. C. Agarwal, "Dependence of human ankle compliance on joint angle", Journal of Biomechanics, vol. 11, no. 4, pp. 177-181, 1978, ISSN: 00219290. DOI: 10.1016/0021-9290(78)90010-6.

[114] C. Tai and C. J. Robinson, "Knee elasticity influenced by joint angle and perturbation intensity", IEEE Transactions on Rehabilitation Engineering, vol. 7 , no. 1, pp. 111-115, 1999, ISSN: 10636528. DOI: 10.1109/86.750561.

[115] P. L. Weiss, R. E. Kearney, and I. W. Hunter, "Position dependence of ankle joint dynamics-II. Active mechanics", Journal of Biomechanics, vol. 19, no. 9, pp. 737-751, 1986, ISSN: 00219290. DOI: 10.1016/0021-9290(86) 90197-1.

[116] E. J. Rouse, L. J. Hargrove, E. J. Perreault, and T. A. Kuiken, "Estimation of human ankle impedance during the stance phase of walking", IEEE Transactions on Neural Systems and Rehabilitation Engineering, vol. 22, no. 4, pp. 870-878, 2014.

[117] H. Lee and N. Hogan, "Time-varying ankle mechanical impedance during human locomotion", IEEE Transactions on Neural Systems and Rehabilitation Engineering, vol. 23, no. 5, pp. 755-764, 2015, ISSN: 15344320. DOI: 10.1109/TNSRE.2014.2346927 
[118] A. L. Shorter, S. Finucane, and E. J. Rouse, "Ankle mechanical impedance during waling in chronic stroke: Preliminary results", IEEE International Conference on Rehabilitation Robotics, pp. 246-251, 2019, ISSN: 19457901. DOI: $10.1109 /$ ICORR.2019.8779436.

[119] L. Lünenburger, G. Colombo, R. Riener, and V. Dietz, "Clinical assessments performed during robotic rehabilitation by the gait training robot Lokomat", Proceedings of the 2005 IEEE 9th International Conference on Rehabilitation Robotics, vol. 2005, pp. 345-348, 2005. DOI: 10.1109/ICORR. 2005.1501116 .

[120] M. R. Tucker, C. Shirota, O. Lambercy, J. Sulzer, and R. Gassert, "Design and Characterization of an Exoskeleton for Perturbing the Knee During Gait", IEEE Transactions on Biomedical Engineering, vol. 64, no. 10, pp. 11, 2017, ISSN: 0018-9294. DOI: 10.1109/TBME.2017.2656130.

[121] K. Yagi, K. Suzuki, and H. Mochiyama, "Human Joint Impedance Estimation with a New Wearable Device Utilizing Snap-Through Buckling of Closed-Elastica", IEEE Robotics and Automation Letters, vol. 3, no. 3, pp. 1506-1513, 2018, ISSN: 23773766. DOI: 10.1109/LRA.2018.2800114.

[122] E. J. Rouse, L. J. Hargrove, E. J. Perreault, M. A. Peshkin, and T. A. Kuiken, "Development of a mechatronic platform and validation of methods for estimating ankle stiffness during the stance phase of walking", Journal of Biomechanical Engineering, vol. 135, no. 8, pp. 1-8, 2013, ISSN: 01480731. DOI: $10.1115 / 1.4024286$

[123] A. Roy, H. I. Krebs, D. J. Williams, C. T. Bever, L. W. Forrester, R. M. Macko, and N. Hogan, "Robot-Aided Neurorehabilitation: A Novel Robot for Ankle Rehabilitation", IEEE Transactions on Neural Systems and Rehabilitation Engineering, vol. 25, no. 3, pp. 569-582, 2009, ISSN: 15344320. DOI: 10.1109/TNSRE.2015.2410773.

[124] J. B. Andersen and T. Sinkjær, "Mobile ankle and knee perturbator", IEEE Transactions on Biomedical Engineering, vol. 50, no. 10, pp. 1208-1211, 2003, ISSN: 00189294. DOI: 10.1109/TBME.2003.816073.

[125] J. S. Sulzer, R. A. Roiz, M. A. Peshkin, and J. L. Patton, "A highly backdrivable, lightweight knee actuator for investigating gait in stroke", IEEE Transactions on Robotics, vol. 25, no. 3, pp. 539-548, 2009, ISSN: 15523098. DOI: 10.1109/TRO.2009.2019788

[126] M. Temel, K. S. Rudolph, and S. K. Agrawal, "Gait recovery in healthy subjects: Perturbations to the Knee motion with a smart Knee Brace", Advanced Robotics, vol. 25, no. 15, pp. 1857-1877, 2011, ISSN: 01691864. DOI: $10.1163 / 016918611 \times 588862$

[127] S. K. Banala, S. K. Agrawal, and J. P. Scholz, "Active Leg Exoskeleton (ALEX) for gait rehabilitation of motor-impaired patients", in IEEE 10th International Conference on Rehabilitation Robotics, 2007, pp. 401-407, ISBN: 1424413206. DOI: $10.1109 /$ ICORR.2007.4428456. 
1 


\title{
Chapter 2
}

\section{Design and evaluation of a lower limb perturbator to estimate hip joint impedance during walking}

S.S. Fricke, R.C. van 't Veld, A. Vallinas Prieto, A.Q.L. Keemink, H. van der Kooij, A.C. Schouten, E.H.F. van Asseldonk

This chapter has been submitted in March 2020.

\begin{abstract}
Robotic gait trainers and assistive devices can overcome neurological disorders affecting walking ability. To optimize assistance during walking the joint mechanical properties should be known. While ankle impedance during walking has been studied previously, hip joint impedance during walking received no attention. Here we developed and evaluated a lower limb perturbator to identify hip joint impedance during treadmill walking. The lower limb perturbator (LOPER) consists of an actuator connected to the upper leg via rods. The LOPER applies force perturbations to a free-hanging leg, while standing on the contralateral leg, with a bandwidth of up to $39 \mathrm{~Hz}$. While walking in minimal impedance mode, the interaction forces of the LOPER are low $(<5 \mathrm{~N})$ and the effect on the walking pattern is smaller than the within-subject variability during normal walking. The apparent hip joint impedance was estimated at three time points during the swing phase. Estimates of the apparent stiffness (average 105-139Nm/rad) and damping (average 3-6Nms/rad) were in the same range as previously reported under static conditions. The developed LOPER has a negligible effect on the walking pattern and allows to identify hip joint impedance during the swing phase. Knowledge on hip joint impedance during walking is relevant for the development of robotic gait trainers, improving training protocols and clinical decision making.
\end{abstract}




\section{$2.1 \quad$ Introduction}

Various robotic gait trainers and assistive devices have been developed to overcome neurological disorders affecting walking ability [1]-3]. Humans can walk in various challenging environments and continuously adjust joint impedance, which describes the mechanical resistance to a perturbation. Neurological disorders, e.g. stroke or spinal cord injury, can affect joint impedance and walking ability, due to symptoms like spasticity and hypertonia [4]-[6]. Consequently, a detailed understanding of joint impedance during walking, in both people with and without neurological disorders, can improve the design and control of robotic gait trainers and assistive devices 7$]-[9]$. Besides, joint impedance assessment in people with neurological disorders can help improve training protocols and clinical decision making 9 .

Joint impedance is estimated by measuring the response to mechanical perturbations applied to the joint by robotic devices and it is often described in terms of joint inertia, damping and stiffness [7, 8], [10- 15. Identification of hip, knee and ankle joint impedance has previously been performed under static conditions, e.g. standing or seated. These experiments showed that joint impedance depends on various factors, including joint angles, joint velocities and muscle activity [1][14, 16 - 19. The results imply that joint impedance must vary during movement. Indeed, for the ankle, a time-varying modulation of joint impedance during walking has been reported [7], 8], [10].

Assessing joint impedance during walking provides additional challenges and requirements for the device applying the mechanical perturbations. An important requirement is that the device should be transparent, i.e. not affect normal walking, when no perturbations are applied. In addition, the device needs to be able to apply the perturbations required for joint impedance estimation. Various devices have been developed to determine ankle or knee joint impedance during walking [20-26]. However, to our knowledge, there are currently no devices which both minimally influence the walking pattern and can determine hip joint impedance during walking.

Here, we 1. developed and evaluated a LOwer limb PERturbator (LOPER) to estimate the apparent hip joint impedance during walking; and 2. obtained a first estimate of apparent hip joint impedance at different time points during the swing phase in healthy volunteers. Apparent hip joint impedance was defined as the estimated impedance at the hip, including effects of gravity, the connection between the device and human, and possible interactions with other joints. Future studies will be aimed at determining 'pure' hip joint impedance which does not depend on these other effects.

\subsection{Requirements}

The device should be able to apply force perturbations, while effects on the walking pattern are negligible. These force perturbations should result in changes in joint angles that can be used to estimate hip joint impedance with system identification techniques. First, a force bandwidth of $10 \mathrm{~Hz}$ is required based on previously deter- 
mined frequency response functions (FRFs) of the hip joint during posture tasks 12. Second, the device should not obstruct the range of motion of the hip joint. Third, we expect that in minimal impedance mode, i.e. when no perturbations are applied, maximal absolute interaction forces of less than $20 \mathrm{~N}$ and root mean square (RMS) interaction forces of less than $10 \mathrm{~N}$ lead to negligible effects on the walking pattern 27]. Additionally, the root-mean-square error (RMSE) between the hip joint angle with and without the device should be lower than the average within-subject variability of the hip joint angle while walking.

\subsection{Methods}

\subsubsection{Design}

LOPER consists of a motor, two carbon fiber rods, an aluminium frame and a brace, which is connected to the left upper leg of a human walking on a treadmill (Figure 2.1). The motor (SMH60, Parker,USA) is attached to the frame of a split-belt treadmill (custom Y-Mill, Forcelink, The Netherlands) with a steel support structure. The first carbon fiber rod (1 in Figure $2.1,0.45 \mathrm{~m}$ long) is rigidly attached to the motor shaft and is connected to the second rod ( 2 in Figure 2.1. $0.84 \mathrm{~m}$ ) via a ball joint linkage. A brace to attach the device to the leg is connected to the rods with an aluminium frame in between. The brace is fixed with Velcro straps on the upper leg, just above the knee. A load cell (FUTEK FSH00086, USA) is implemented between the second rod and the aluminium frame to measure interaction forces.

We have chosen a design where the actuator is not placed on the human to minimize the additional load on the user. Moreover, the choice to place the load cell close to the human limb can result in lower interaction forces and a lower influence of the device on the walking pattern compared with placing the sensor farther away from the user 28 . Finally, the design allows for sufficient freedom of movement in both sagittal and frontal planes. The chosen rod lengths do not limit the range of motion in the sagittal plane for a person walking on the treadmill. The connection between the aluminium frame and the brace allows for free rotation of the leg in the sagittal plane. The ball joint linkage between the two rods allows for freedom of movement in the frontal plane.

\subsubsection{Hardware and software setup}

The system to control the LOPER and record the force data is composed of one master PC (Linux) and six slave devices:

1: a servo drive unit (MOOG MSD 3200 Servo Drive, USA) that controls the velocity of the motor

2: an amplifier (MOOG PC CB79047-401A_HCU, USA) for the signals from the load cell

3-6: four Beckhoff modules (1x Beckhoff EK1100, 2x Beckhoff EL3008 and 1x Beckhoff EL4134, Beckhoff Automation GmbH, Germany) which are used 


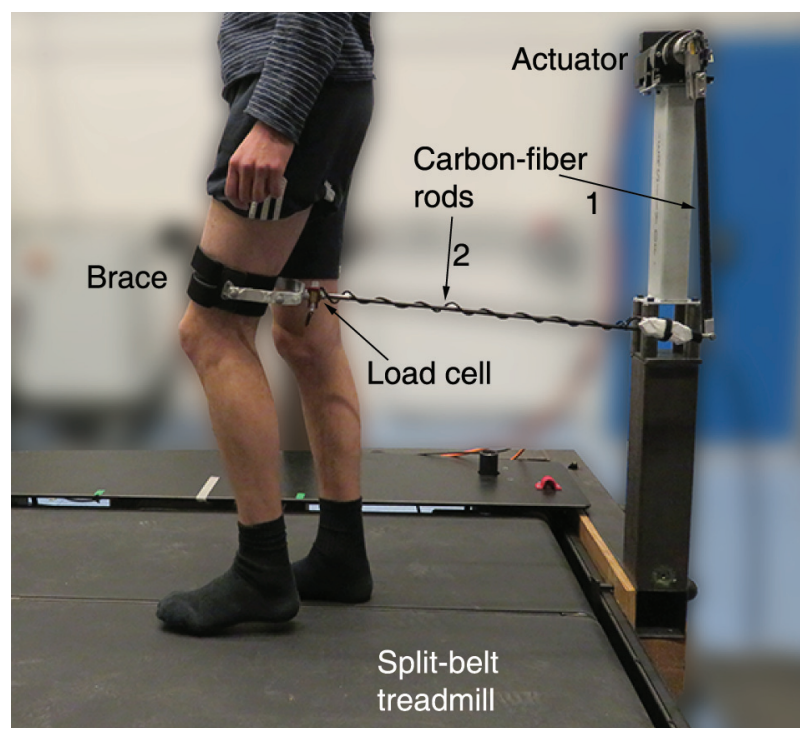

Figure 2.1: Overview of LOPER (LOwer limb PERturbator) rigidly attached to the frame of a split-belt treadmill. The actuator is connected to the left upper leg of a user with two carbon-fiber rods and a brace with an aluminium frame in between. The load cell is placed close to the user (between carbon rod and aluminium frame) and measures the interaction forces between the user and the LOPER.

to acquire 3 degrees of freedom (DoFs) ground reaction forces and moments from the treadmill and to send a synchronization signal to the motion capture system.

This network of one master and six slave devices is controlled using the EtherCAT real-time control protocol, which runs through EtherLab. On the master PC, a compiled Simulink model (Matlab 2016b, Mathworks, US) runs at $1000 \mathrm{~Hz}$ by EtherLab, using TestManager and DLS (Beckhoff Automation GmbH, Germany), to control the device, acquire and save the data from the slaves.

\subsubsection{Control}

The device is controlled by a modified admittance controller $\left(H_{C}\right.$, Figure 2.2 designed to minimize the interaction forces $\left(F_{i n}\right)$ in absence of perturbations and track the desired interaction forces $\left(F_{d}\right)$ of the applied perturbations. The admittance model generates the input $\left(\dot{\theta}_{d}\right)$ to the black-box velocity controller, implemented in the servo drive unit.

To minimize interaction forces, an admittance control law with low virtual impedance is implemented. However, a regular admittance controller with low inertia and damping yielded unstable behavior, while interacting with humans. Therefore, the $K_{a} s+1$ term is included in the numerator of $H_{C}$, which behaves similarly to the acceleration feed-forward described by Keemink et al. 29, and prevents interaction instability. We included this term in the admittance model, 


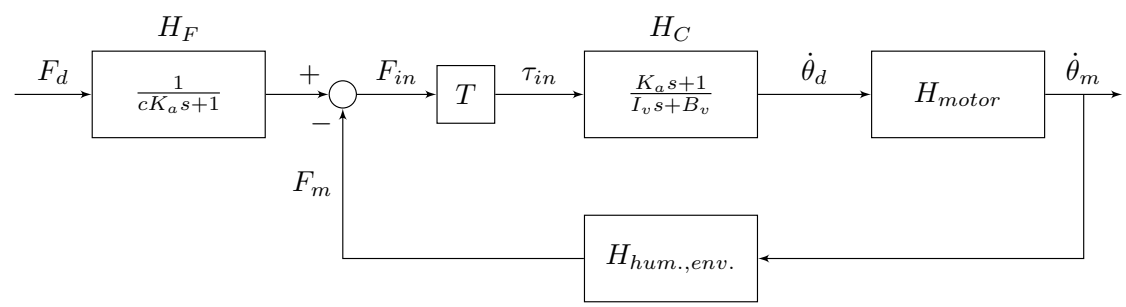

Figure 2.2: Overview of the control scheme. The admittance model $\left(H_{C}\right)$ computes the desired angular velocity $\left(\dot{\theta}_{d}\right)$ based on the torque input $\left(\tau_{i n}\right)$. This torque is obtained by multiplying the force input $\left(F_{i n}\right)$ with the moment arm in T. $H_{\text {motor }}$ represents the motor and rod dynamics and $H_{\text {hum.,env. }}$ are the post sensor dynamics, e.g. human and brace. $F_{m}$ represents the interaction force measured by the load cell and $F_{d}$ is the desired perturbation force, which is first low-pass filtered through $\mathrm{H}_{F}$.

because the black-box low level controller of the motor does not accept feed-forward inputs. The perturbations are low-pass filtered $\left(H_{F}\right)$ to prevent overshoot, at the expense of frequency bandwidth of the perturbation. When tuning the parameters, first low values for $I_{v}$ and $B_{v}$ were chosen resulting in fast dynamics. Then, $K_{a}$ and $c$ were chosen to achieve a stable system with sufficient bandwidth. The following values were chosen for the parameters: $c=0.5, K_{a}=0.017, I_{v}=0.2, B_{v}=3$.

\subsubsection{Safety}

Five features are implemented to assure safety of the user. First, a PVA (position, velocity, acceleration) limiter prevents that the chosen safety bounds for velocity $( \pm 4.71 \mathrm{rad} / \mathrm{s})$ and acceleration $( \pm 500 \mathrm{rad} / \mathrm{s})$ are exceeded $[3]$. Close to the position safety bound $( \pm 1.22 \mathrm{rad})$, the PVA limiter limits the motor velocity resulting in a constant deceleration. Beyond this position bound, the velocity is limited to $0 \mathrm{~m} / \mathrm{s}$ in the direction away from the neutral position. Second, the outputs from the sensors (encoders and load cells) are continuously checked and the motor is switched off directly when a threshold for either position $( \pm 1.31 \mathrm{rad})$, velocity $( \pm 6.28 \mathrm{~m} / \mathrm{s})$ or motor torque $(120 \mathrm{Nm})$ is reached. These boundaries are slightly larger than the PVA limiter bounds to prevent that the motor turns off each time a slightly larger angle is reached. Third, when the servo drive unit detects an angle larger than $\pm 1.34 \mathrm{rad}$ the motor is switched off directly. Fourth, two emergency buttons are placed close to both experimenter and user, which directly switch off the motor when pressed. Fifth, users wear a safety harness to prevent falls.

\subsubsection{Ethics}

The experiments were approved by the ethics committee of the Electrical Engineering, Mathematics and Computer Science faculty of the University of Twente (approval number: RP 2019-83). All participants gave informed consent prior to the experiments. 


\subsubsection{Device performance in static situations}

The performance of the LOPER was evaluated in two tests in which a participant was standing on his fully extended right leg. The left leg, connected to the LOPER, was lifted from the ground and relaxed, i.e. free-hanging. In the first test, the force bandwidth was evaluated using a force perturbation of filtered white noise for $60 \mathrm{~s}$ (cut-off frequency $60 \mathrm{~Hz}$, peak-to-peak amplitude of $60 \mathrm{~N}$ ). In the second test, step responses were evaluated for force perturbations with several amplitudes $(20,40$ and $60 \mathrm{~N}$ in forward direction, each applied 10 times).

\subsubsection{Device performance during walking}

Five participants ( 4 male, 1 female, $26.4 \pm 1.3$ years, length $1.71 \pm 0.09 \mathrm{~m}$, weight $68.4 \pm 11.5 \mathrm{~kg}$ ) without any self-reported impairments in their lower limbs walked two times four minutes on the treadmill to assess the performance of the minimal impedance mode of the device. Participants walked on the treadmill at $0.5 \mathrm{~m} / \mathrm{s}$ while following a metronome (36 strides/min.) to keep the stride frequency as constant as possible. The low walking speed was chosen for all experiments as it is relevant for clinical applications in people with neurological disorders. In the first trial, participants walked without the device. In the second trial, participants walked with the LOPER in minimal impedance mode.

\subsubsection{Assessing joint impedance during swing phase}

Nine participants (6 male, 3 female, $26.1 \pm 1.2$ years, length $1.73 \pm 0.14 \mathrm{~m}$, weight $64.9 \pm 12.1 \mathrm{~kg}$ ) without any self-reported impairments in their lower limbs were included in the joint impedance assessment experiments. To estimate joint impedance during the swing phase, force perturbations were applied at three onset times: 50, 175 and 300ms after toe-off. Rectangular pulses with a pulse width of $100 \mathrm{~ms}$ and an amplitude of 40N (forward) were applied as perturbation in all cases. In order to time the perturbations appropriately, a phase detection algorithm was used to detect toe-off based on the vertical ground reaction forces. The perturbation experiments were split into six trials, two trials for each perturbation onset time, with the trial order randomized for each participant. Each trial lasted four minutes, with a walking speed of $0.5 \mathrm{~m} / \mathrm{s}$ and a stride frequency of 36 strides $/ \mathrm{min}$. For each trial, the perturbations were applied randomly after every 3-5 strides during the swing phase of the left leg at the specified perturbation onset time.

\subsubsection{Data recording and processing}

During all experiments, the load cell measured the interaction forces. For the experiments during walking, $3 \mathrm{DoF}$ ground reaction forces and moments were recorded from the treadmill. A motion capture system (Qualysis AB, Sweden) recorded the movements of the participants and the brace of LOPER. Eight Oqus $600+$ cameras were placed around the treadmill and marker movements were recorded at $128 \mathrm{~Hz}$. The synchronisation signal, that was send from the LOPER setup to the motion capture system, was recorded at $1024 \mathrm{~Hz}$ using an analog to digital conversion board. Two markers were placed on the brace of the device to 
be able to calculate the angle of the brace and the direction of the applied forces. 32 markers were placed on bony landmarks and body segments of the participants to record kinematics of feet, lower legs, upper legs, pelvis and trunk. All data was processed in Matlab 2018b. In the following sections and figures, a positive sign indicates a force (of the device) in forward direction, i.e. walking direction, and a hip flexion angle/torque.

\section{Device performance in static situations}

The FRF of the force controller was determined by dividing the following spectral densities:

$$
H_{F_{d} t o F_{m}}=\frac{H_{F} T H_{C} H_{\text {motor }} H_{\text {hum. }, \text { env. }}}{1+T H_{C} H_{\text {motor }} H_{\text {hum. }, \text { env. }}}=\frac{S_{F_{m} F_{d}}}{S_{F_{d} F_{d}}}
$$

where $F_{d}$ is the input perturbation and $F_{m}$ the measured interaction force. Welch averaging with a Hanning window (size: 5000 samples, overlap: 50 samples) was used to calculate these spectral densities. The bandwidth of the force controller was calculated as the $-3 \mathrm{~dB}$ point of the FRF.

Step responses were averaged across the ten repetitions to reduce noise. Rise time was calculated for each perturbation amplitude and defined as the time needed by the measured interaction force to rise from 10 to $90 \%$ of the steady state response. Percentage overshoot was determined relative to the steady state response.

\section{Device performance during walking}

Gait phase estimates, calculated based on ground reaction forces and moments, were used to cut the LOPER data into strides, i.e. a full gait cycle. For the walking trials without perturbations, interaction forces between the device and the human were averaged over all strides within a participant. The RMS interaction forces and maximal absolute interaction forces were determined for each participant and averaged across participants.

Hip joint angles were determined based on the motion capture data. The measured marker positions were filtered in Matlab 2018b with a $4^{\text {th }}$ order zerophase $40 \mathrm{~Hz}$ low-pass Butterworth filter. In OpenSim 4.0 the gait2392 model was used to perform inverse kinematics. The resulting hip joint angles were cut into strides similar to the LOPER data. For each data point of a stride, we determined whether the data point was 1.5 times the interquartile range below the first quartile or above the third quartile (of all strides at the same percentage of the gait cycle). If these limits were exceeded for more than $20 \%$ of the data points of a stride, this stride was considered an outlier and discarded. After outlier removal, strides were averaged within each participant. For each participant, the RMSE between the average joint trajectories for the trials with and without the device were calculated. The RMSE was compared to the average intra subject variability $\left(\mathrm{ISV}_{\text {ave }}\right)$ defined as twice the average standard deviation between strides within a participant 27]:

$$
I S V_{\text {ave }}=\frac{2}{n \text { Part. }} \sum_{\text {iPart. }=1}^{n \text { Part. }} \sigma_{\text {iPart }}
$$


We assumed that effects of the device were negligible if the RMSE between the trial without and with the device was smaller than the $\mathrm{ISV}_{\text {ave }}$ for the trial without the device 27.

\section{Assessing joint impedance during the swing phase}

Hip joint angles were determined and processed as described in the previous paragraph. Interaction forces were filtered with the same $4^{\text {th }}$ order zero-phase $40 \mathrm{~Hz}$ low-pass Butterworth filter, after which the interaction forces were resampled to $128 \mathrm{~Hz}$. These interaction forces were converted into torques applied to the hip based on the moment arm of LOPER. This moment arm was determined using the angle between leg and rod, and the distance between the hip joint and attached brace, based on the scaled (participant-specific) OpenSim model.

Both, hip joint angles and applied torques, were cut into strides. For each perturbed step, the most similar unperturbed stride was found, based on the RMSE between the perturbed and unperturbed strides for the last 25ms before the perturbation onset time, and subtracted from the perturbed trial. For the last perturbation onset time $(300 \mathrm{~ms})$, strides with a swing time shorter than $550 \mathrm{~ms}$ $(300 \mathrm{~ms}+250 \mathrm{~ms}$ analysis window) were removed, to avoid the inclusion of the stance phase in the analysis. Again, outliers were removed as described above, but only taking into account the range of data points that was used for joint impedance estimation ( $25 \mathrm{~ms}$ before to $250 \mathrm{~ms}$ after the perturbation).

After removing outliers, average hip joint angles and applied torques were used to estimate hip joint impedance. The analysis of each perturbation onset time included data from $25 \mathrm{~ms}$ before to $250 \mathrm{~ms}$ after the perturbation. We assumed that the hip joint impedance can be estimated using a $2^{\text {nd }}$ order model:

$$
\Delta \tau=I \ddot{\Delta} \theta+B \dot{\Delta} \theta+K \Delta \theta
$$

with $\Delta \tau$ the difference in the measured torque between perturbed and unperturbed steps, $\Delta \theta$ the difference in the hip angle, $I$ the apparent inertia of the leg, $B$ the apparent hip joint damping and $K$ the apparent hip joint stiffness. This $2^{\text {nd }}$ order model was chosen as it performed well in describing the ankle joint during walking 8 and experimental data during standing has shown that the FRF of the hip resembles a $2^{\text {nd }}$ order system 12 . The parameters of this model (I, B, K) were estimated by simulating the time response of the $2^{\text {nd }}$ order model and minimizing the sum squared difference.

\section{Sensitivity analysis of estimated joint impedance results}

The effect of varying the model parameters on the response was evaluated, to validate the parameter estimation results and to determine the influence of each parameter on the model response. Each parameter was changed separately by $\pm 20 \%$ of the best fit and used to simulate the time response of the $2^{\text {nd }}$ order model for each of these parameter variations. 


\subsection{Results}

\subsubsection{Device performance in static situations}

We designed and evaluated the LOPER, which can be used to apply force perturbations to the left upper leg. A bandwidth of $39 \mathrm{~Hz}$ was found for the FRF (input $F_{d}$, output $F_{m}$ ) when applying perturbations to a free-hanging leg (Figure 2.3 which is much higher than the required bandwidth of $10 \mathrm{~Hz}$. Step responses show rise times of $9.7-10.3 \mathrm{~ms}$ for 20,40 and $60 \mathrm{~N}$ perturbations (Figure 2.4). An overshoot of $29-32 \%$ of the steady state responses was found. The steady state responses did not fully reach the desired values, e.g. the steady state response for the $40 \mathrm{~N}$ perturbation was $36 \mathrm{~N}$ (Figure 2.4).

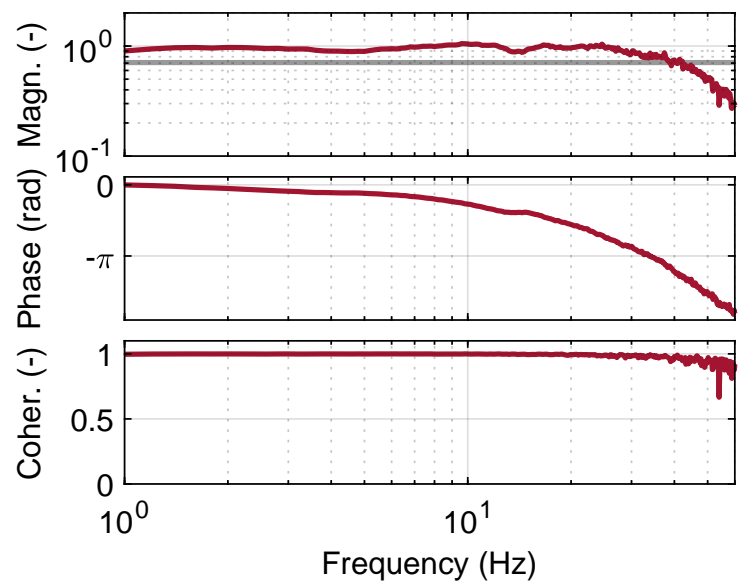

Figure 2.3: FRF of the control scheme shown in Figure 2.2 (input $F_{d}$, output $F_{m}$ ) while the device was attached to a free-hanging leg of a participant who was standing on his fully extended contralateral leg. Results for one participant are shown. A bandwidth of $39 \mathrm{~Hz}$ was found, indicated by the grey line at $-3 d B(0.71)$.

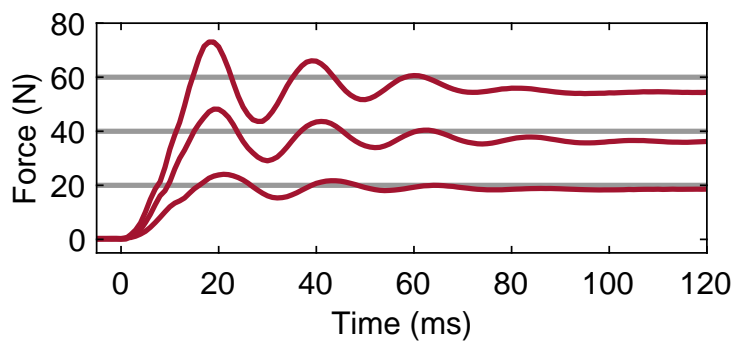

Figure 2.4: Step responses (averaged over 10 responses) in one participant with three amplitudes $(20,40,60 N)$ that were applied to a free-hanging leg in forward direction. The participant was standing on his fully extended contralateral leg. The grey lines represent the desired steady state values. 


\subsubsection{Device performance during walking}

To evaluate the performance of the device during walking, participants walked without the LOPER, and with the LOPER attached to their left upper leg in minimal impedance mode. In line with the requirements, low interaction forces were found in minimal impedance mode (Figure 2.5 top). The average ( \pm standard deviation across participants) RMS interaction forces were $2.00 \pm 0.20 \mathrm{~N}$ and the maximal absolute interaction forces were $4.63 \pm 0.79 \mathrm{~N}$. The largest interaction forces were found at the end of the stance phase/beginning of the swing phase, which can be attributed to the high acceleration of the leg at this portion of the gait cycle.

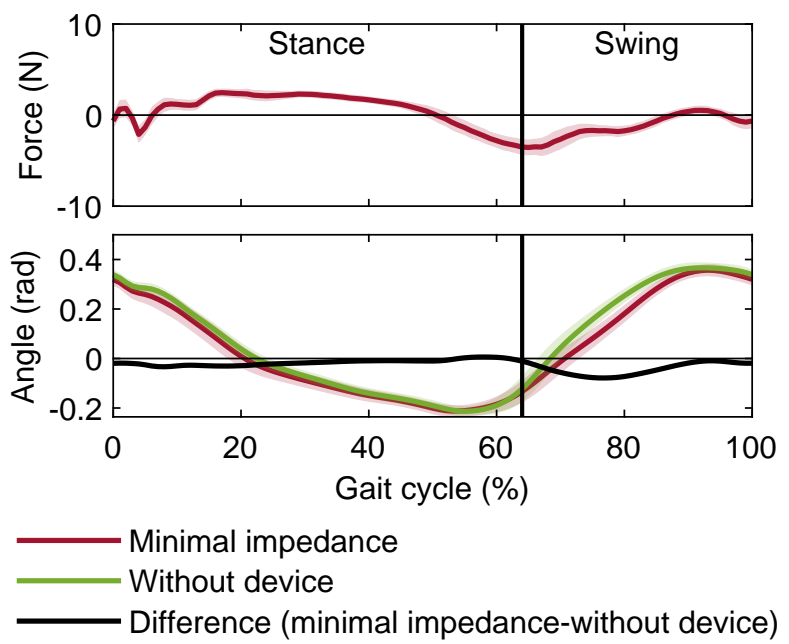

Figure 2.5: Interaction forces (top) in minimal impedance mode and joint angles (bottom) for the left leg while walking in minimal impedance mode and without the device for one representative participant. The positive axis shows a force in forward direction (top) and a flexion angle (bottom). The shaded areas illustrate the standard deviation across the strides. Left heel strike occurred at $0 \%$ of the gait cycle.

The differences in the hip angles between walking with the device in minimal impedance mode and without the device were negligible when averaged over the complete gait cycle (Figure 2.5 bottom). The average RMSE between the trial without the device and the minimal impedance trial was $0.030 \pm 0.010 \mathrm{rad}$ which is lower than the $\mathrm{ISV}_{\text {ave }}(0.047 \mathrm{rad})$ for walking without the device. The largest differences between the trial with and without the device were found during the beginning/mid swing, probably due to the larger interaction forces at the beginning of the swing phase (Figure 2.5).

\subsubsection{Assessing joint impedance during swing phase}

To assess apparent hip joint performance during walking, participants walked six times on the treadmill, while perturbations were applied with the LOPER at 
three onset times during the swing phase. All participants performed two trials for each perturbation onset time. However, for one participant, one trial (175ms) is missing due to data acquisition issues, leaving one trial for analysis. For the last perturbation onset time $(300 \mathrm{~ms}), 38 \%$ of the total amount of steps for all participants had to be removed, because swing times were shorter than $550 \mathrm{~ms}$. Still, at least 30 steps were included for the analysis for each participant at each perturbation onset time.

The applied perturbations led to more hip flexion during and after the perturbation irrespective of when it was applied (Figure 2.6 and 2.7). No differences between the unperturbed and perturbed joint angles were observed before the perturbation, the largest difference occurred around $150 \mathrm{~ms}$ after the perturbation onset. About $400 \mathrm{~ms}$ after the perturbation onset, the perturbed joint angle trajectories returned to the unperturbed ones.

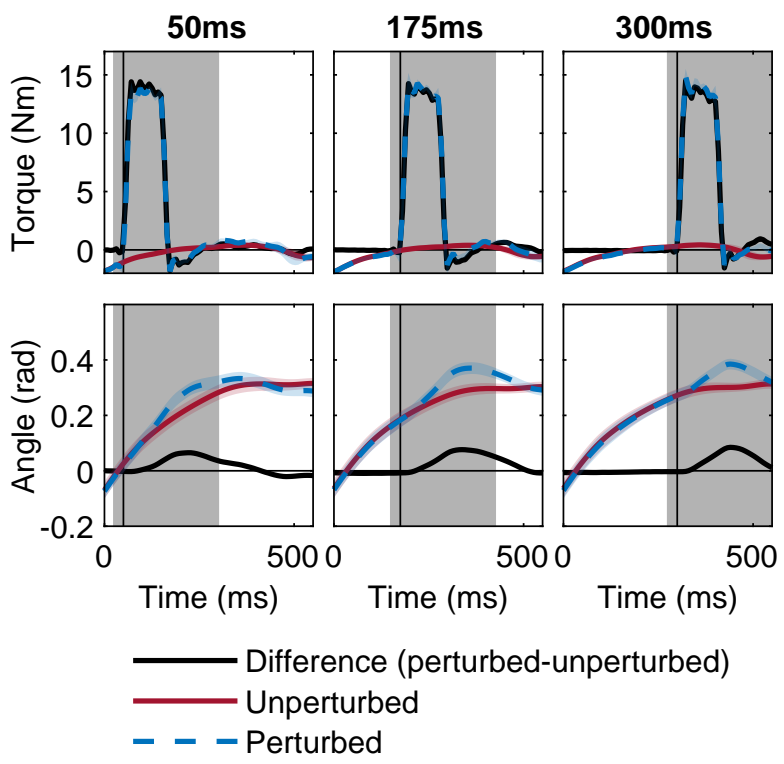

Figure 2.6: Measured torques and hip joint angles for the perturbed and unperturbed steps during the swing phase (Oms is at the start of the swing phase). The positive axes indicate a flexion torque/angle. Each column shows the results for one perturbation onset point and the analyzed window is indicated with a grey box. The vertical line indicates the beginning of the perturbation. The differences between the perturbed and unperturbed torques and hip joint angles were used to estimate joint impedance and calculate the model angle shown in Figure 2.7.

The difference between the measured angle and the model angle calculated with the estimated parameters was low (Figures 2.7 and 2.8, maximum RMSE of $\left.2.24 \cdot 10^{-3} \mathrm{rad}\right)$. For all parameters (I, B, K), some variability was found between participants and perturbation onset times (Figure 2.8). Especially the results for the apparent hip joint stiffness $(K)$ varied between participants: on average the 
lowest apparent stiffness was found for a perturbation onset at $175 \mathrm{~ms}$, while two participants had the largest apparent stiffness at that point. In addition, a slight decrease in inertia (I) was found for later perturbation onsets. The damping (B) was lowest for the perturbation at $50 \mathrm{~ms}$ and slightly higher for later perturbation onset times.

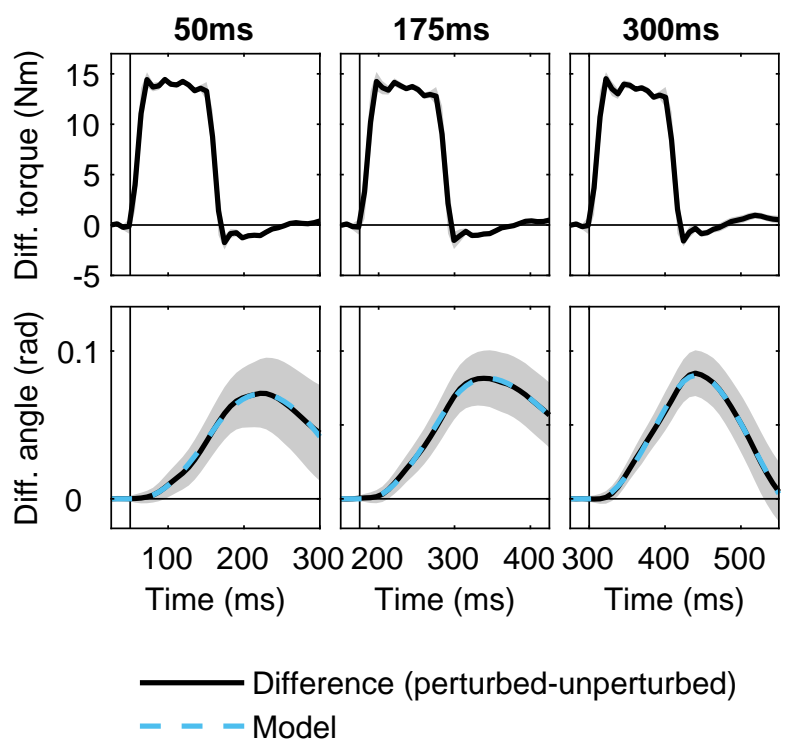

Figure 2.7: Differences in the torques and hip joint angles (between perturbed and unperturbed steps), and model hip joint angles. The vertical line indicates the beginning of the perturbation. On the $x$-axis, Oms is at the start of the swing phase and only the analyzed window (grey box in Figure 2.6) is shown for each perturbation onset point.

\subsubsection{Sensitivity analysis of estimated joint impedance results}

To assess sensitivity of the model hip joint angles to changes in the identified parameters, each parameter was varied by $\pm 20 \%$ of the best fit. Each parameter influenced the response to the torque perturbations in a unique way (Figure 2.9. The error between measured and model joint angle clearly increased when the parameters were varied compared with the best fit. The inertia influenced the whole response, while the effect of damping and stiffness was mostly visible between 150 and $250 \mathrm{~ms}$ after the start of the perturbation.

\subsection{Discussion}

The goal of our study was two-fold: 1. to develop and evaluate a lower limb perturbator (LOPER) to estimate the apparent hip joint stiffness during walking; 


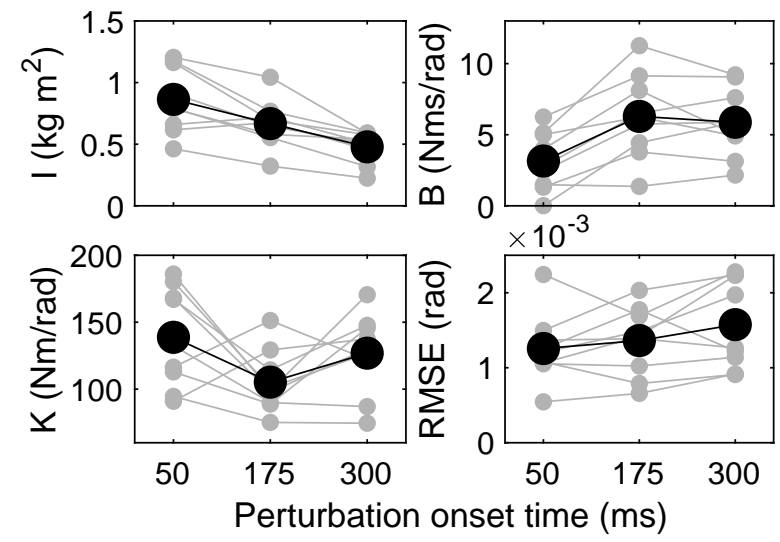

Figure 2.8: Estimated apparent inertia (I), damping (B), and stiffness (K) of the hip, and RMSE between the measured and model hip angle. The black line with markers indicates the average across all participants. Each grey line with markers shows the results for one participant.

and 2. to obtain a first estimate of hip joint impedance at different time points during the swing phase in healthy volunteers.

\subsubsection{Performance of the device}

The developed device, LOPER, fulfills all requirements needed to identify apparent hip joint impedance during the swing phase. LOPER obtained a force bandwidth of $39 \mathrm{~Hz}$ when forces were applied to a free-hanging leg while the participant stood on the contralateral leg. The bandwidth is much higher than the reported bandwidth of the hip impedance $(<10 \mathrm{~Hz})[12$ ] and therefore sufficient for estimating hip mechanical properties. The mechanical design, where forces are transmitted to the upper leg using rods, did not restrict the motions of the hip during walking. Moreover, due to the high bandwidth and low interaction forces, LOPER had a negligible effect on the walking pattern. This was also proven by the small average differences in joint angles between walking without and with LOPER in minimal impedance mode. Walking with the LOPER resulted in a more gradual swing, i.e. less acceleration in the beginning of the swing phase. In the future, controllers that take into account the cyclic behaviour of walking could be used to further increase transparency $[30]$ and reduce this effect.

\subsubsection{Joint impedance during swing phase}

We obtained first estimates of apparent hip joint impedance at different time points during the swing phase by applying perturbations with LOPER. The magnitudes of the estimated apparent hip joint stiffness and damping (average stiffness for the different onset times $105-139 \mathrm{Nm} / \mathrm{rad}$ and damping $3-6 \mathrm{Nms} / \mathrm{rad}$ ) were in the range of previously found values in static positions (average stiffness $50-450 \mathrm{Nm} / \mathrm{rad}$ and damping 6-19Nms/rad [11, [12]). The values we found are on the lower side of 


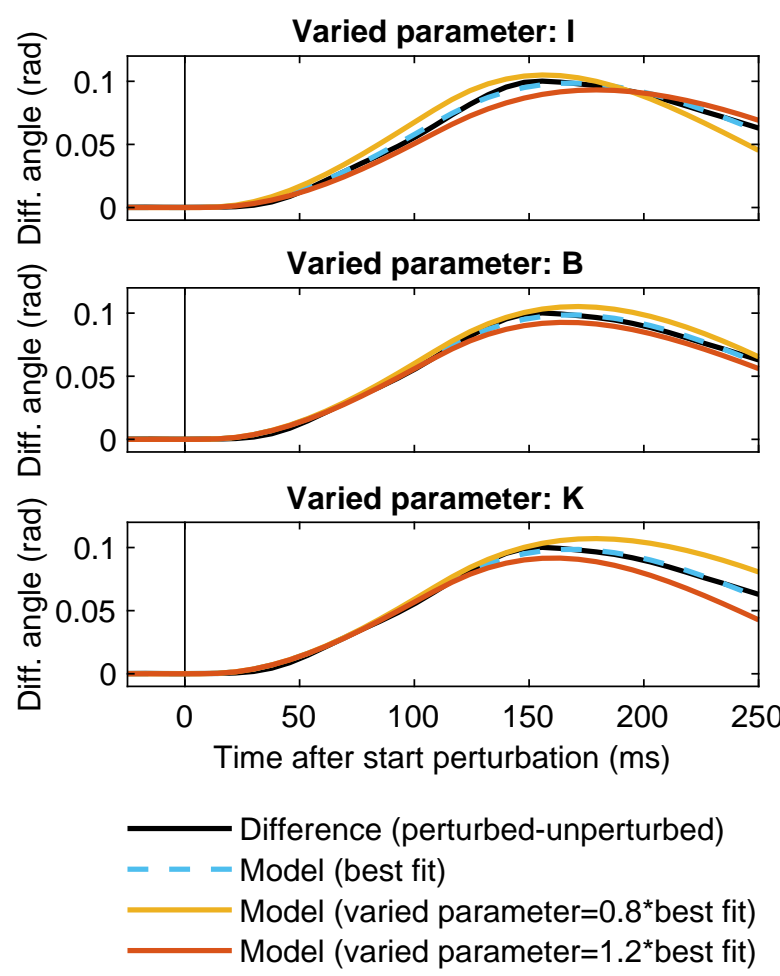

Figure 2.9: Effects of varying the parameters of the $2^{\text {nd }}$ order model on the model joint angles for one representative condition (one participant, perturbation at 175ms). The vertical lines indicate the beginning of the perturbation. Each parameter $(I, B, K)$ was varied separately, using a factor 0.8 or 1.2 times the best fit of the parameter (Figure 2.8).

this range which could be expected as during the swing phase muscle activity will be low. Participants in previous studies were asked to keep the leg in a specific position [12] or apply a force [1] which can result in a larger muscle activation. Our study is the first study that determined apparent hip joint impedance during walking. A direct comparison with the impedance estimates obtained in static situations is hampered by the fact that joint angles and muscle activity are different during walking 11$]-13$.

Comparing estimates for the inertia with other studies is difficult, as the estimated inertia depends on the experimental setup and body configuration. Various body segments can influence the estimated inertia. This is probably also the reason for the large differences in inertia estimates between our study (average 0.48 to $\left.0.86 \mathrm{kgm}^{2}\right)$ and a previous study $\left(3.5 \mathrm{kgm}^{2}\right) 31$. In the previous study, measurements were performed on a hanging leg while the participant was wearing a brace that kept the knee in an extended position [31]. The estimated inertia was close to the inertia of the entire leg based on an anatomical model $\left(3.5 \mathrm{kgm}^{2}\right) 32$. In con- 
trast to this, our estimated inertia during walking was more similar to the inertia of the upper leg $\left(0.34 \mathrm{kgm}^{2}\right.$ for the average weight and length of our participants) than to the inertia of the whole leg $\left(2.76 \mathrm{kgm}^{2}\right)$ 32. During swing phase, knee stiffness is relatively low and therefore our inertia estimates are mainly influenced by the inertia of the upper leg.

Our results illustrated the challenges of determining time-varying behaviour of the hip joint impedance. The response time after applying a pulse-like perturbation at the upper leg is longer than at the ankle [8], mainly due to the large inertia of the upper-leg compared to the foot. Consequently, large time windows are required to identify each parameter (inertia, damping, stiffness) from the response. This limits the ability to assess fast time-varying behaviour, as our parameter estimation approach assumed parameters to be constant within the analysis window.

\subsubsection{Limitations}

Limitations of the LOPER are the relatively large overshoots for the step responses and that the desired steady-state values are not fully reached. This can be a limiting factor for studies that aim to get a more detailed understanding of joint impedance and underlying physiology. In this case, either the control algorithm of the device needs to be improved or the exact influence of these limitations needs to be further investigated.

Another limitation of our study is that the obtained estimates are not estimates of the 'pure' hip joint impedance, because the estimates were influenced by the dynamics of the entire leg. For example, the parameters could be influenced by changes of the knee angle, knee stiffness and/or biarticular muscles spanning the hip and knee joint. In addition, the dynamics of the connection between the human and the device, and the effect of gravity were not modelled and might have influenced impedance estimates. Initial rough estimates based on the strap stiffness from 12 indicate that hip joint stiffness might have been underestimated by about $15 \mathrm{Nm} / \mathrm{rad}$ due to the properties of the connection. In addition, initial rough estimates based on an anatomical model from 32 and our measured joint angles show that the contribution of the gravitational stiffness to the estimated apparent hip joint stiffness was approximately $35 \mathrm{Nm} / \mathrm{rad}$. However, more detailed analyses are needed to determine the exact magnitude of these different contributions to the estimated apparent hip joint impedance.

\subsubsection{Future directions}

In the future, the device and analysis methods should be extended to address the above mentioned limitations in order to determine 'pure' hip joint impedance, e.g. not including dynamics of the entire leg, as well as knee joint impedance. For this purpose, another actuator has to be added that is able to apply perturbations to both lower and upper leg simultaneously. Multi-input multi-output (MIMO) techniques should be used to determine hip and knee joint impedance as well as possible interactions between the joints, e.g. due to biarticular muscles [12].

Furthermore, the measurements of the hip and knee joint impedance should be extended to the stance phase, which has already been done for the ankle joint [8], 
10. This results in additional requirements for the device and analysis methods. First, larger forces need to be applied during stance phase to result in similar changes in joint angles, e.g. due to weight bearing and an expected larger joint stiffness in the stance phase 33 . Second, perturbations during stance phase might have larger influences on the walking pattern, e.g. disturbing balance.

The presented methods allow for identifying joint impedance in people with neurological disorders, e.g. stroke, spinal cord injury, to gain a better understanding of their impaired walking ability. However, applying these methods in people with neurological disorders will pose additional challenges. People with neurological impairments can have problems following the metronome leading to a larger gait variability [34. This larger gait variability can increase the difficulty of timing the perturbations consistently and could influence the responses to perturbations. In addition, impairments, e.g. spasticity or hypertonia, can result in an increased joint stiffness [5], 35]. Therefore, larger forces might be needed to bring about clear changes in joint angles in people with neurological disorders.

More insight about mechanical properties of the hip under different walking conditions could be achieved by determining joint impedance at higher walking speeds. However, currently it is difficult to estimate fast time-varying changes. At a low walking speed $(0.5 \mathrm{~m} / \mathrm{s})$ the impedance estimates for the three perturbation onset times were determined based on analysis time windows that overlapped. The overlap could not be prevented as the required time windows for the analysis were relatively large $(300 \mathrm{~ms})$ compared to the total swing phase (about 550ms). For higher walking speeds, swing time is shorter and this issue becomes even more evident. Previously, more advanced system identification algorithms were developed to determine time-varying joint impedance [36]. These methods were used for slow time-varying behaviour $(0.05 \mathrm{~Hz})$ of the wrist joint. Further developments are needed to possibly make these algorithms suitable for estimations of time-varying joint impedance during walking.

\subsection{Conclusion}

We developed a lower limb perturbator (LOPER) with a high bandwidth and negligible effects on the walking pattern that can be used to estimate hip joint impedance during the swing phase. With LOPER, we were able to obtain first estimates of apparent hip joint impedance during walking.

Although there are still challenges to overcome, methods to determine joint impedance during walking are relevant for the development of robotic gait trainers and assistive devices for people with neurological impairments. Moreover, assessment of joint impedance in people with neurological impairments can be used to develop training protocols and track progress during therapy.

\section{References}

[1] A. Esquenazi, M. Talaty, and A. Jayaraman, "Powered Exoskeletons for Walking Assistance in Persons with Central Nervous System Injuries: A 
Narrative Review", PM and R, vol. 9, no. 1, pp. 46-62, 2017, ISSN: 19341482. DOI: $10.1016 / \mathrm{j} \cdot \mathrm{pmrj} \cdot 2016.07 .534$.

[2] G. Morone, S. Paolucci, A. Cherubini, D. De Angelis, V. Venturiero, P. Coiro, and M. Iosa, "Robot-assisted gait training for stroke patients: Current state of the art and perspectives of robotics", Neuropsychiatric Disease and Treatment, vol. 13, pp. 1303-1311, 2017, ISSN: 11782021. DOI: 10.2147/NDT.S114102

[3] J. Meuleman, E. H. van Asseldonk, G. van Oort, H. Rietman, and H. van der Kooij, "LOPES II - Design and Evaluation of an Admittance Controlled Gait Training Robot with Shadow-Leg Approach", IEEE Transactions on Neural Systems and Rehabilitation Engineering, vol. 24, no. 3, pp. 352-363, 2016, ISSN: 15344320. DOI: 10.1109/TNSRE.2015.2511448

[4] V. Dietz and T. Sinkjaer, "Spastic movement disorder: impaired reflex function and altered muscle mechanics", Lancet Neurology, vol. 6, no. 8, pp. 725733, Aug. 2007, ISSN: 14744422. DOI: 10.1016/S1474-4422(07)70193-X.

[5] A. Roy, H. I. Krebs, C. T. Bever, L. W. Forrester, R. F. Macko, and N. Hogan, "Measurement of passive ankle stiffness in subjects with chronic hemiparesis using a novel ankle robot", Journal of Neurophysiology, vol. 105, no. 5, pp. 2132-2149, 2011, ISSN: 0022-3077. DOI: 10.1152/ jn.01014.2010.

[6] H. Lee, T. Patterson, J. Ahn, D. Klenk, A. Lo, H. I. Krebs, and N. Hogan, "Static ankle impedance in stroke and multiple sclerosis: A feasibility study", Proceedings of the Annual International Conference of the IEEE Engineering in Medicine and Biology Society, EMBS, pp. 8523-8526, 2011, ISSN: 1557170X. DOI: 10.1109/IEMBS.2011.6092103.

[7] A. L. Shorter, S. Finucane, and E. J. Rouse, "Ankle mechanical impedance during waling in chronic stroke: Preliminary results", IEEE International Conference on Rehabilitation Robotics, pp. 246-251, 2019, ISSN: 19457901. DOI: $10.1109 /$ ICORR.2019.8779436.

[8] E. J. Rouse, L. J. Hargrove, E. J. Perreault, and T. A. Kuiken, "Estimation of human ankle impedance during the stance phase of walking", IEEE Transactions on Neural Systems and Rehabilitation Engineering, vol. 22, no. 4, pp. 870-878, 2014.

[9] S. Maggioni, A. Melendez-Calderon, E. H. F. van Asseldonk, V. KlamrothMarganska, L. Lünenburger, R. Riener, and H. van der Kooij, Robot-aided assessment of lower extremity functions: A review, 2016. DOI: 10.1186/ s12984-016-0180-3.

[10] H. Lee and N. Hogan, "Time-varying ankle mechanical impedance during human locomotion", IEEE Transactions on Neural Systems and Rehabilitation Engineering, vol. 23, no. 5, pp. 755-764, 2015, ISSN: 15344320. DOI: 10.1109/TNSRE. 2014.2346927. 
[11] H.-Y. Huang, A. Arami, I. Farkhatdinov, D. Formica, and E. Burdet, "The influence of posture, applied force and perturbation direction on hip joint viscoelasticity", ArXiv e-prints, pp. 1-7, 2019. arXiv: 1908.07800.

[12] B. Koopman, E. H. F. van Asseldonk, and H. van der Kooij, "Estimation of Human Hip and Knee Multi-Joint Dynamics Using the LOPES Gait Trainer", IEEE Transactions on Robotics, vol. 32, no. 4, pp. 920-932, 2016, ISSN: 15523098. DOI: 10.1109/TRO.2016.2572695.

[13] D. Ludvig, M. Plocharski, P. Plocharski, and E. J. Perreault, "Mechanisms contributing to reduced knee stiffness during movement", Experimental Brain Research, vol. 235, no. 10, pp. 1-12, 2017, ISSN: 14321106. DOI: $10.1007 / \mathrm{s} 00221-017-5032-2$.

[14] L. Q. Zhang, G. Nuber, J. Butler, M. Bowen, and W. Z. Rymer, "In vivo human knee joint dynamic properties as functions of muscle contraction and joint position", Journal of Biomechanics, vol. 31, no. 1, pp. 71-76, 1997, ISSN: 00219290. DOI: 10.1016/S0021-9290(97)00106-1.

[15] R. E. Kearney and I. W. Hunter, "System Identification of Human Joint Dynamics", Critical reviews in biomedical engineering, vol. 18, no. 1, pp. 5587, 1990, ISSN: 0278-940X. DOI: 10.1007/s13398-014-0173-7.2. arXiv: arXiv:1011.1669v3.

[16] P. L. Weiss, R. E. Kearney, and I. W. Hunter, "Position dependence of ankle joint dynamics-I. Passive mechanics", Journal of Biomechanics, vol. 19, no. 9, pp. 727-735, 1986, ISSN: 00219290. DOI: 10.1016/0021-9290(86) 90196-X.

[17] G. L. Gottlieb and G. C. Agarwal, "Dependence of human ankle compliance on joint angle", Journal of Biomechanics, vol. 11, no. 4, pp. 177-181, 1978, ISSN: 00219290. DOI: 10.1016/0021-9290(78)90010-6.

[18] C. Tai and C. J. Robinson, "Knee elasticity influenced by joint angle and perturbation intensity", IEEE Transactions on Rehabilitation Engineering, vol. 7, no. 1, pp. 111-115, 1999, ISSN: 10636528. DOI: 10.1109/86.750561.

[19] P. L. Weiss, R. E. Kearney, and I. W. Hunter, "Position dependence of ankle joint dynamics-II. Active mechanics", Journal of Biomechanics, vol. 19, no. 9, pp. 737-751, 1986, ISSN: 00219290. DOI: 10.1016/0021-9290(86) 90197-1.

[20] M. R. Tucker, C. Shirota, O. Lambercy, J. Sulzer, and R. Gassert, "Design and Characterization of an Exoskeleton for Perturbing the Knee During Gait", IEEE Transactions on Biomedical Engineering, vol. 64, no. 10, pp. 11, 2017, ISSN: 0018-9294. DOI: 10.1109/TBME.2017.2656130

[21] K. Yagi, K. Suzuki, and H. Mochiyama, "Human Joint Impedance Estimation with a New Wearable Device Utilizing Snap-Through Buckling of Closed-Elastica", IEEE Robotics and Automation Letters, vol. 3, no. 3, pp. 1506-1513, 2018, ISSN: 23773766. DOI: 10.1109/LRA.2018.2800114. 
[22] E. J. Rouse, L. J. Hargrove, E. J. Perreault, M. A. Peshkin, and T. A. Kuiken, "Development of a mechatronic platform and validation of methods for estimating ankle stiffness during the stance phase of walking", Journal of Biomechanical Engineering, vol. 135, no. 8, pp. 1-8, 2013, ISSN: 01480731. DOI: $10.1115 / 1.4024286$

[23] A. Roy, H. I. Krebs, D. J. Williams, C. T. Bever, L. W. Forrester, R. M. Macko, and N. Hogan, "Robot-Aided Neurorehabilitation: A Novel Robot for Ankle Rehabilitation", IEEE Transactions on Neural Systems and Rehabilitation Engineering, vol. 25, no. 3, pp. 569-582, 2009, ISSN: 15344320. DOI: $10.1109 /$ TNSRE. 2015.2410773.

[24] J. B. Andersen and T. Sinkjær, "Mobile ankle and knee perturbator", IEEE Transactions on Biomedical Engineering, vol. 50, no. 10, pp. 1208-1211, 2003, ISSN: 00189294. DOI: 10.1109/TBME.2003.816073.

[25] J. S. Sulzer, R. A. Roiz, M. A. Peshkin, and J. L. Patton, "A highly backdrivable, lightweight knee actuator for investigating gait in stroke", IEEE Transactions on Robotics, vol. 25, no. 3, pp. 539-548, 2009, ISSN: 15523098. DOI: $10.1109 /$ TRO.2009.2019788

[26] M. Temel, K. S. Rudolph, and S. K. Agrawal, "Gait recovery in healthy subjects: Perturbations to the Knee motion with a smart Knee Brace", Advanced Robotics, vol. 25, no. 15, pp. 1857-1877, 2011, ISSN: 01691864. DOI: $10.1163 / 016918611 \times 588862$

[27] J. H. Meuleman, E. H. F. van Asseldonk, and H. van der Kooij, "The effect of directional inertias added to pelvis and ankle on gait", Journal of NeuroEngineering and Rehabilitation, vol. 10, no. 40, pp. 1-12, 2013.

[28] D. Zanotto, T. Lenzi, P. Stegall, and S. K. Agrawal, "Improving transparency of powered exoskeletons using force/torque sensors on the supporting cuffs", IEEE International Conference on Rehabilitation Robotics, no. June, 2013, ISSN: 19457898. DOI: 10.1109/ICORR.2013.6650404

[29] A. Q. Keemink, H. van der Kooij, and A. H. Stienen, "Admittance control for physical human-robot interaction", International Journal of Robotics Research, vol. 37, no. 11, pp. 1421-1444, 2018, ISSN: 17413176. DOI: 10 . $1177 / 0278364918768950$.

[30] W. van Dijk, B. Koopman, E. H. F. van Asseldonk, and H. van der Kooij, "Improving the transparency of a rehabilitation robot by exploiting the cyclic behaviour of walking", IEEE International Conference on Rehabilitation Robotics, pp. 1-8, 2013, ISSN: 19457898. DOI: 10.1109/ICORR.2013. 6650393 .

[31] H.-Y. Huang, I. Farkhatdinov, A. Arami, M. Bouri, and E. Burdet, "Cabledriven robotic interface for lower limb neuromechanics identification", ArXiv e-prints, pp. 1-12, 2019. arXiv: 1908.02689

[32] D. A. Winter, Biomechanics and Motor Control of Human Movement: Fourth Edition. Hoboken, New Jersey: John Wiley \& Sons, Inc., 2009, pp. 1370, ISBN: 9780470398180 . DOI: 10.1002/9780470549148 
[33] H. Lee, E. J. Rouse, and H. I. Krebs, "Summary of Human Ankle Mechanical Impedance during Walking", IEEE Journal of Translational Engineering in Health and Medicine, vol. 4, no. September, 2016, ISSN: 21682372. DOI: $10.1109 /$ JTEHM. 2016.2601613.

[34] M. Roerdink, C. J. Lamoth, J. Van Kordelaar, P. Elich, M. Konijnenbelt, G. Kwakkel, and P. J. Beek, "Rhythm perturbations in acoustically paced treadmill walking after stroke", Neurorehabilitation and Neural Repair, vol. 23, no. 7, pp. 668-678, 2009, ISSN: 15459683. DOI: $10.1177 /$ 1545968309332879 .

[35] E. de Vlugt, J. H. de Groot, K. E. Schenkeveld, J. H. Arendzen, F. C. T. van der Helm, and C. G. M. Meskers, "The relation between neuromechanical parameters and Ashworth score in stroke patients.", Journal of neuroengineering and rehabilitation, vol. 7, p. 35, 2010, ISSN: 1743-0003. DOI: $10.1186 / 1743-0003-7-35$.

[36] M. van de Ruit, G. Cavallo, J. Lataire, F. C. van der Helm, W. Mugge, J. W. van Wingerden, and A. C. Schouten, "Revealing Time-Varying Joint Impedance with Kernel-Based Regression and Nonparametric Decomposition", IEEE Transactions on Control Systems Technology, vol. 28, no. 1, pp. 224-237, 2020, ISSN: 15580865. DOI: 10.1109/TCST.2018.2881664. 


\title{
Chapter 3
}

\section{Performance-based adaptive assistance for diverse subtasks of walking in a robotic gait trainer: Description of a new controller and preliminary results}

S.S. Fricke*, C. Bayón*, E. Rocon, H. van der Kooij, E.H.F. van Asseldonk (*these authors contributed equally to this work)

This chapter was published in Proceedings of the IEEE RAS and EMBS International Conference on Biomedical Robotics and Biomechatronics, 2018.

\begin{abstract}
Robotic gait training is a promising tool for gait rehabilitation in people with neurological disorders. Personalizing the applied robotic assistance based on objective assessments is expected to further improve therapy outcomes. Here we present a novel controller which automatically adjusts robotic assistance based on users' performance for diverse subtasks of gait (e.g. foot clearance, prepositioning). The resulting amount of assistance applied by this automatically-tuned (AT) controller could serve as an assessment tool, e.g. for monitoring progress during therapy. However, during training, factors such as walking speed and/or partial body weight support (PBWS) might vary. The performance criteria of an assessment tool should not depend on these factors to result in a reliable assessment. We took a first step in evaluating the potential of the AT controller as an assessment tool. Ten healthy participants walked in the LOPES II robotic gait trainer, with assistance applied by the AT controller, at various walking speeds and levels of PBWS. We evaluated the effect of walking speed and PBWS on performances for all subtasks and the amount of assistance that was applied by the AT controller. Performances for all subtasks were dependent on the amount of PBWS. For two of five subtasks, performances were also influenced by walking speed. For most subtasks, most participants did not receive assistance as they were performing well. The assistance applied by the AT controller cannot directly be used as an
\end{abstract}


assessment tool, e.g. to compare between participants. However, it has potential to be used for monitoring progress within patients when the amount of PBWS and the walking speed are kept constant. Future studies will be focused on further testing the AT controller in people with neurological disorders to determine its potential as a therapeutic and monitoring tool.

\subsection{Introduction}

Robotic gait trainers have been developed in recent decades to improve gait rehabilitation after neurological disorders such as stroke, spinal cord injury (SCI) or cerebral palsy (CP) [1]-[5]. Robot-assisted gait training (RAGT) shows some promising advantages compared to conventional therapy, since it can be applied in an intensive, controlled, repetitive and task-specific way. This can lead to neural plasticity and improve motor learning [6. However, RAGT should be further optimized to increase its effectiveness, enhance motor learning and improve recovery [5], 6]. Personalized assistance, based on objective measurements might improve therapy outcomes after RAGT. In addition, we expect that robotic gait trainers can be valuable tools not only for therapy, but also to include objective assessments of gait in RAGT.

The minimal amount of assistance that a user needs to walk in a robotic gait trainer can be used as a measure of walking function [7]. For example, a high amount of assistance would be an indication of walking deficiencies. Maggioni et al. 8 developed an algorithm that automatically adapted assistance based on users' performance. However, this algorithm adjusts assistance for 30 bins of the gait cycle and does not take into account functional aspects of gait. Meuleman et al. [2] implemented a controller that is related to functional aspects of gait (also called subtasks). Robotic assistance can be provided for these specific subtasks (e.g. foot clearance, stability during stance, step length), which are often related to impairments after neurological disorders. This controller might also be used to assess walking function. However, in the first implementation [2], the physical therapist has to set the amount of assistance required for each subtask, which means that the assistance is not automatically adjusted based on users' performance within a session.

This contribution presents a novel controller that automatically adapts robotic assistance for diverse subtasks of gait based on users' performances for each subtask. Walking speed and partial body weight support (PBWS) often change during therapy and might be confounding factors when the assistance is used as an outcome measure. As a first step to determine the feasibility of the AT controller as an assessment tool, we determine the effects of walking speed and PBWS on subtask performance as well as on the assistance applied by the AT algorithm. The controller is evaluated using the LOPES II gait trainer [2], but it could also be used for other robotic gait trainers. 


\subsection{Methods}

\subsubsection{Robotic device}

LOPES II is a treadmill-based, admittance-controlled robotic gait trainer that has eight actuated degrees of freedom (hip flexion/extension, hip abduction/adduction, knee flexion/extension, pelvis forward/backward and pelvis mediolateral) to control the motion of the lower limbs and pelvis $[2$. The device is currently controlled through a graphical user interface in which the operator can manually adjust the level of robotic assistance for several subtasks of gait presented in 2] (i.e. leading limb angle, trailing limb angle, foot clearance, stability during stance, prepositioning and weight shift). For each subtask, the assistance provided by LOPES II can be scaled from no support (minimal impedance mode) to $100 \%$ support. These settings are currently constant during a therapy session unless the therapist changes it.

\subsubsection{Performance-based adaptive controller for diverse subtasks of walking}

The new performance-based automatically-tuned (AT) controller is shown in Figure 3.1. It is focused on movements in the sagittal plane since this is the dominant plane of motion for human walking. Movements were evaluated for hip and knee joints, and classified into five subtasks of walking for each leg [2]: foot clearance, stability during stance, prepositioning, and leading and trailing limb angle (Table 3.1). The AT controller adjusts the robotic assistance per subtask based on user's performance during previous steps. In this study the previous three steps are used for the evaluation.

Table 3.1: Subtasks, supported movements, key point locations and upper and lower bounds that were used to adjust assistance levels (see also Figure 3.1 and Equations 3.1 and 3.2).

\begin{tabular}{|c|c|c|c|c|}
\hline Subtask & $\begin{array}{l}\text { Supported move- } \\
\text { ment (gain) }\end{array}$ & Key point location & $\begin{array}{l}\text { Lower } \\
\text { bound } \\
\text { (deg.) }\end{array}$ & $\begin{array}{l}\text { Upper } \\
\text { bound } \\
\text { (deg.) }\end{array}$ \\
\hline Foot clearance & Knee flexion $(-1)$ & $\begin{array}{l}\text { Maximal knee flex- } \\
\text { ion }\end{array}$ & 4.91 & 8.19 \\
\hline $\begin{array}{l}\text { Stability during } \\
\text { stance }\end{array}$ & $\begin{array}{l}\text { Knee extension } \\
\text { stance }(+1)\end{array}$ & $\begin{array}{l}\text { Knee weight accep- } \\
\text { tance }\end{array}$ & 2.86 & 4.77 \\
\hline Prepositioning & $\begin{array}{l}\text { Knee extension } \\
\text { and hip flexion late } \\
\text { swing }(+1)\end{array}$ & $\begin{array}{l}\text { Maximal knee ex- } \\
\text { tension late swing }\end{array}$ & 3.44 & 5.73 \\
\hline $\begin{array}{l}\text { Leading } \quad \operatorname{limb} \\
\text { angle }\end{array}$ & Hip flexion $(-1)$ & Maximal hip flexion & 2.86 & 4.77 \\
\hline $\begin{array}{l}\text { Trailing } \quad \text { limb } \\
\text { angle }\end{array}$ & Hip extension $(+1)$ & $\begin{array}{l}\text { Maximal hip exten- } \\
\text { sion }\end{array}$ & 2.46 & 4.09 \\
\hline
\end{tabular}

Subtask-based errors were determined by evaluating the differences between a 


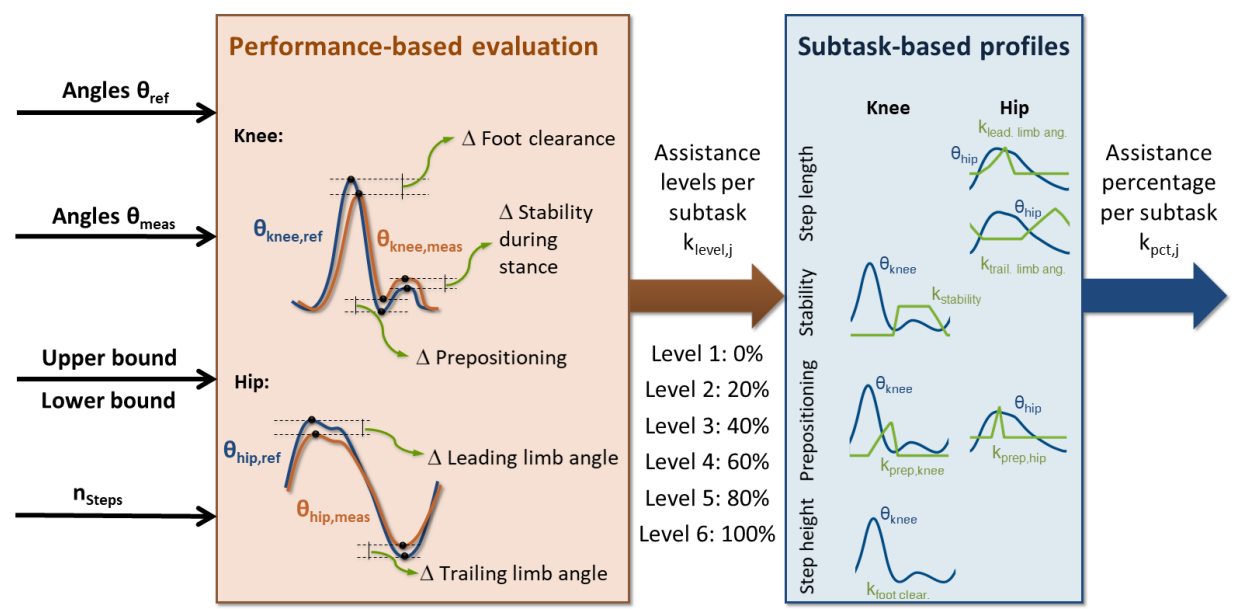

Figure 3.1: Overview of the AT controller. User's performance is evaluated based on the differences between measured ( $\left.\theta_{\text {meas }}\right)$ and reference $\left(\theta_{\text {ref }}\right)$ joint angles at some 'key points' of the gait cycle (deviations at black dots in 'Performance-based evaluation' box). The errors per subtask are compared to thresholds (upper and lower bound according to Equations 3.2) after a specific number of steps ( $\left.n_{\text {Steps }}\right)$. Based on this, a new level of assistance ( $k_{\text {level, }, j}$ from 0\% to 100\%) is applied for each subtask. Specific assistance profiles are applied by the robotic gait trainer for each subtask (green lines in 'Subtaskbased profiles' box). The assistance is tuned for each leg separately.

reference pattern and the measured joint angles at specific key points of the gait cycle for each leg separately ('Performance-based evaluation' box in Figure 3.1):

$$
\text { Error }_{j}=G_{j} \frac{\sum_{i=1}^{n}\left(\theta_{\text {meas }, j}\right)_{i}-\sum_{i=1}^{n}\left(\theta_{\text {ref }, j}\right)_{i}}{n}
$$

where $j$ is the subtask that is evaluated, and $\theta_{\text {ref }}$ and $\theta_{\text {meas }}$ (expressed in degrees) are the reference and measured angles at the subtask-specific key points (Table 3.1. These angles are determined for each step $i$ and averaged over a specific number of steps $\left(n_{\text {Steps }}=3\right.$ in this study). $G_{j}$ is a subtask-specific gain (Table 3.1. Gains are negative or positive depending on the supported movement (flexion and extension respectively). Users' performance was penalized if they did not reach the reference pattern, but not if they overcame it. We assumed that exceeding the reference trajectory would not be detrimental. For example, for the foot clearance subtask, not enough knee flexion (i.e. too much extension) is a common problem after stroke and SCI and results in a positive error for our controller. Assistance was increased if this error was too large (see next paragraph), to prevent that the toes hit the floor during swing phase. In contrast to this, too much knee flexion was not penalized as this also prevents that the toes prematurely hit the ground.

The error for each subtask $j$ was compared to a selected lower and upper bound in order to update the robotic assistance per subtask $\left(k_{j}\right)$. The lower and upper bounds (Table 3.1) are based on pilot experiments in healthy participants walking in LOPES II. The assistance can be affected in different ways (Equations 3.2): 
First, if the error is lower than the lower bound, the assistance is decreased. Second, if the error is larger than the upper bound, the assistance is increased. If the error is in between the lower and upper bound, assistance remains constant. The robotic assistance is classified into six discrete levels of support (from 0 to $100 \%$ with steps of $20 \%$ ). We considered this number of levels enough to provide the needed assistance. Also it is easier to interpret the outcomes when the controller is used for assessment. Assistance levels per subtask go either one level up or down or remain constant based on Equations 3.2 .

$$
\begin{aligned}
& \text { Error }_{j}<\text { LowerBound }_{j} \Rightarrow \downarrow k_{\text {level }, j} \\
& \text { Error }_{j}>\text { UpperBound }_{j} \Rightarrow \uparrow k_{\text {level }, j} \\
& \text { else } \Rightarrow \text { constant } k_{\text {level }, j}
\end{aligned}
$$

The subtask-specific control is achieved by using the different assistance profiles (see green lines in the 'Subtask-based profiles' box of Figure 3.1). The peak amplitude is changed based on the chosen assistance level. Any combination of assistance profiles is possible. The resulting assistance is the sum of all different assistance profiles.

\subsubsection{Preliminary implementation}

Walking speed and PBWS are two parameters that often change during gait training in people with neurological disorders. These variations could become confounding factors for assessment, and hamper reliable measurements of walking function 9], 10. It is crucial to understand the effects of walking speed and PBWS on the behaviour of our AT controller in order to evaluate the feasibility of the controller for the assessment of walking function. The following sections present the implementation of the previously described AT controller with ten healthy participants who walked in the LOPES II gait trainer at various walking speeds and levels of PBWS.

\section{Participants}

Ten volunteers without neurological, muscular or orthopaedic impairments participated in this study (seven male, three female, weight $72.79 \pm 12.11 \mathrm{~kg}$; height $1.80 \pm 0.07 \mathrm{~m}$; and age $26.3 \pm 2.36$ years). The protocol was approved by the Medical Ethical Committee Twente (The Netherlands). All participants received written and verbal information about the experiment and gave written informed consent.

\section{Protocol}

LOPES II was fitted to each user and attached to the user's lower legs and feet. Subsequently, the harness was fixed to the pelvis and trunk to provide fall protection and PBWS.

The protocol consisted of 10 trials (T0-T9) (Table 3.2). Participants were asked to walk as normal as possible during all trials. First, participants walked at $0.3 \mathrm{~m} / \mathrm{s}$ without robotic assistance and no PBWS to become used to walking in LOPES II (T0). The order of the other trials (T1-T9) was randomized for 
each participant, considering every possible combination of three walking speeds $(0.2,0.4$ and $0.6 \mathrm{~m} / \mathrm{s})$ and three levels of PBWS $(0 \%, 20 \%$ and $40 \%)$. In all of these trials, participants walked for 1.5 minutes in minimal impedance mode (no assistance), followed without breaks by 3 minutes in which the AT controller was turned on. Subjects did not receive any information on the provided assistance to minimize bias.

Table 3.2: Overview of trials to test the effects of walking speed and PBWS on the behaviour of the adaptive controller. All trials started with walking in minimal impedance mode. For T1-T9 this was followed by 3 minutes walking with the AT controller.

\begin{tabular}{l|l|l|l|lr} 
Trial & Speed $(\mathrm{m} / \mathrm{s})$ & PBWS $(\%)$ & $\begin{array}{l}\text { Minimal } \\
\text { impedance } \\
\text { (min.) }\end{array}$ & $\begin{array}{l}\text { AT } \\
\text { troller } \\
\text { (min.) }\end{array}$ & $\begin{array}{r}\text { on- } \\
\text { on }\end{array}$ \\
\hline T0 & 0.3 & 0 & 5 & - \\
\hline T1 & 0.2 & 0 & 1.5 & 3 \\
T2 & 0.2 & 20 & 1.5 & 3 \\
T3 & 0.2 & 40 & 1.5 & 3 \\
\hline T4 & 0.4 & 0 & 1.5 & 3 \\
T5 & 0.4 & 20 & 1.5 & 3 \\
T6 & 0.4 & 40 & 1.5 & 3 \\
\hline T7 & 0.6 & 0 & 1.5 & 3 \\
T8 & 0.6 & 20 & 1.5 & 3 \\
T9 & 0.6 & 40 & 1.5 & 3
\end{tabular}

\section{Analysis}

Hip and knee joint angles, step length, users' errors and received robotic assistance were analysed for the above-mentioned subtasks of gait, and compared between the various walking speeds and amounts of PBWS.

We conducted statistical analyses using IBM SPSS Statistics v.23 (IBM, United States). We applied two-way repeated measures ANOVA to evaluate differences in the errors per subtask for the SPEED (3 levels) and PBWS (3 levels) during minimal impedance mode (no assistance). This period without assistance was chosen to avoid influences of the robotic assistance on natural users' errors. Pairwise comparisons with Bonferroni corrections were applied for all main effects and interactions that were significant for the repeated measures ANOVA.

\subsection{Results}

As the experiments were performed on healthy participants, similar results were found for both legs. Therefore, in this section, we only show the results for the participants' right legs. 


\subsubsection{Effects of PBWS on kinematics}

Since the reference gait trajectories for LOPES II were only adjusted based on walking speed but not for PBWS [11, we focused on differences in kinematics between the different levels of PBWS. Maximal hip flexion and extension angles decreased when the amount of PBWS increased while walking at $0.4 \mathrm{~m} / \mathrm{s}$ (hip joint in Figure 3.2. Similar results were found for the other walking speeds. These decrements in maximal hip flexion and extension resulted in a shorter step length for larger amounts of PBWS (Figure 3.3). For the knee joint, we found a decrease in the knee flexion peak for an increased amount of PBWS (Figure 3.2). In contrast, more knee extension at heel strike was achieved for larger amounts of PBWS.

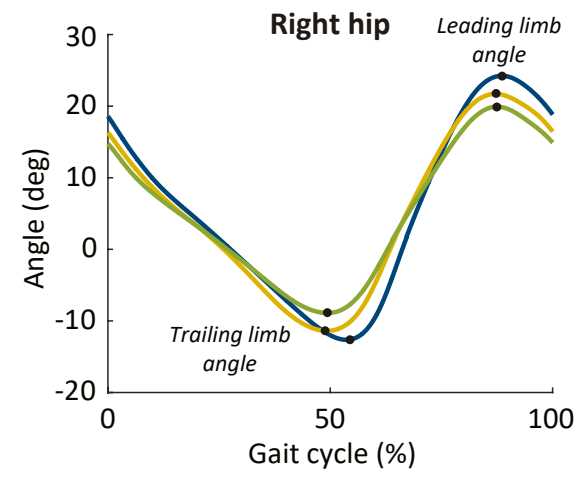

$0 \%$

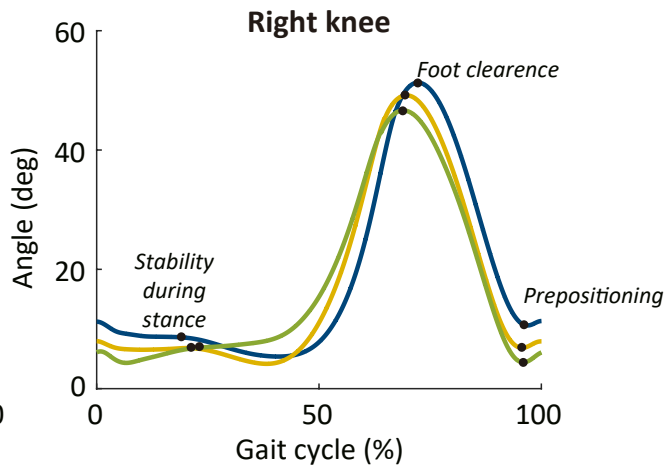
$40 \%$ PBWS

Figure 3.2: Effects of various amounts of PBWS on mean joint angles for ten healthy users walking at $0.4 \mathrm{~m} / \mathrm{s}$ when no robotic assistance was provided. Black dots indicate key points for each subtask.

\section{Right step length}

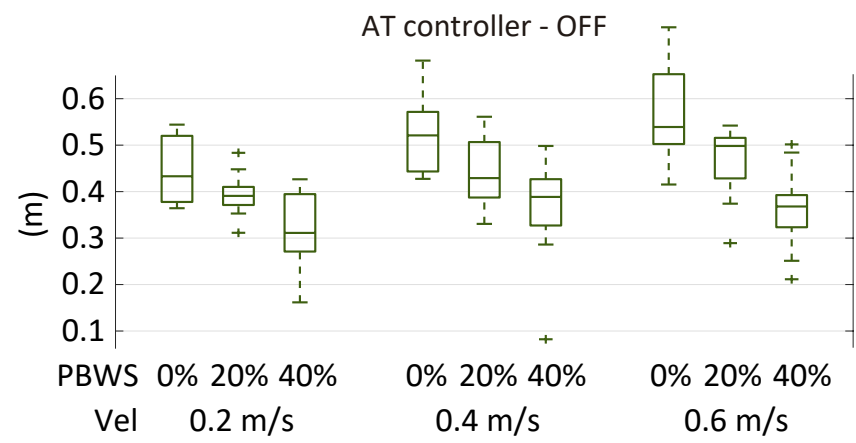

Figure 3.3: Effects of various amounts of PBWS on right step length for ten healthy participants when no robotic assistance was provided. 


\section{Right trailing limb angle}
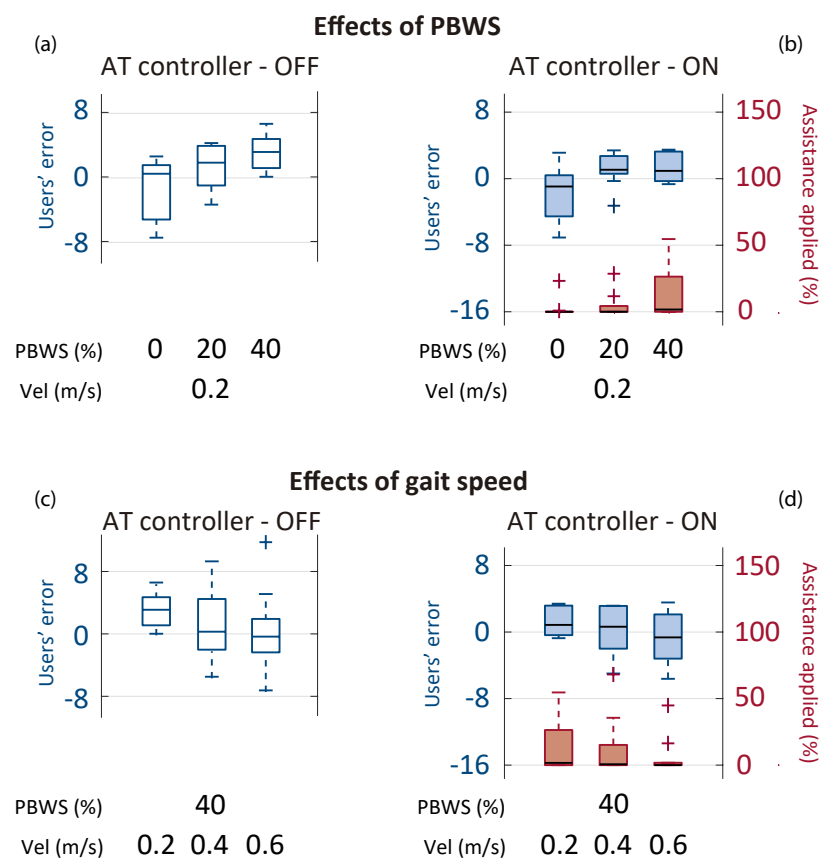

Figure 3.4: Examples of errors for right trailing limb angle determined with Equation 3.2 in ten healthy users walking in two situations: i) when no robotic assistance is applied (unfilled blue boxes, (a) and (c)), and ii) when the performance-based controller applied robotic assistance (filled blue boxes, (b) and (d)). The applied robotic assistance is represented in \% (red boxes). (a) and (b) represent an example of the effects of various levels of PBWS when the speed is maintained constant. Meanwhile, (c) and (d) show the effects of various walking speeds when the PBWS is kept constant.

\subsubsection{Effects of walking speed and PBWS on the performance without robotic assistance}

The changes in joint angles when no robotic assistance was applied also led to changes in the errors calculated with Equation 3.1. Errors for leading and trailing limb angle (hip flexion and extension) and foot clearance (knee flexion) increased when PBWS increased. In contrast, errors decreased for prepositioning and stability during stance (knee extension) when more PBWS was applied. Two-way repeated measures ANOVA confirmed significant effects of PBWS for all subtaskbased errors (Table 3.3). Moreover, two of five subtasks also showed significant effects of walking speed. As an example, the unfilled boxplots of Figure 3.4 show the changes of user's errors for trailing limb angle when no robotic assistance was provided in two different cases: i) constant speed, varying PBWS (Figure 3.4 a), and ii) constant PBWS, varying speed (Figure 3.4 c).

Pairwise comparisons showed the following significant differences $(p<0.05)$ for 
Table 3.3: P-values for the two-way repeated measures ANOVA on performances without assistance for each subtask of gait. * indicates significant differences with p-value $<0.05$, and ${ }^{* *}$ significant differences with p-value $<0.001$.

\begin{tabular}{l|l|l|l} 
Subtask & SPEED & PBWS & SPEED*PBWS \\
\hline Foot clearance & 0.799 & $0.018^{*}$ & 0.525 \\
Stability during & $0.023^{*}$ & $0.021^{*}$ & 0.085 \\
stance & & $0.004^{*}$ & \\
Prepositioning & 0.261 & $0.000^{* *}$ & 0.748 \\
Leading limb & 0.451 & & 0.057 \\
angle & & $0.000^{* *}$ & 0.714 \\
Trailing limb & $0.003^{*}$ & & \\
angle & & &
\end{tabular}

PBWS: between $0 \%$ and 20\%: leading limb angle $(\mathrm{p}=0.001)$ and trailing limb angle $(\mathrm{p}=0.040)$, foot clearance $(\mathrm{p}=0.019)$ and stability during stance $(\mathrm{p}=0.032)$; between $0 \%$ and $40 \%$ : leading limb angle $(\mathrm{p}=0.001)$ and trailing limb angle $(\mathrm{p}=0.033)$, foot clearance $(\mathrm{p}=0.038)$ and prepositioning $(\mathrm{p}=0.016)$; and between $20 \%$ and $40 \%$ : leading limb angle $(\mathrm{p}=0.003)$ and prepositioning $(\mathrm{p}=0.024)$. In case of walking speed, significant differences $(\mathrm{p}<0.05)$ in performances were found between $0.2 \mathrm{~m} / \mathrm{s}$ and $0.6 \mathrm{~m} / \mathrm{s}$ for trailing limb angle $(\mathrm{p}=0.033)$; and between 0.2 $\mathrm{m} / \mathrm{s}$ and $0.4 \mathrm{~m} / \mathrm{s}$ for stability during stance $(\mathrm{p}=0.005)$ and trailing limb angle $(\mathrm{p}=0.040)$.

\subsubsection{Effects of walking speed and PBWS on the assistance applied by the AT controller}

As expected, healthy participants did not receive robotic assistance in most of the cases because their error was lower than the threshold (lower bound). For this reason, medians of robotic assistance were close to zero for most trials (see the example of robotic assistance received for trailing limb angle, red boxplots in Figure 3.4.

Only the errors of some subtasks in a limited number of participants and conditions was larger than the selected upper bounds. In these cases, the controller increased the robotic assistance that was provided to the users (see red boxplots in Figure 3.4 to improve gait performance per subtask (blue boxplots in Figure 3.4). This robotic assistance was affected by changes of PBWS (e.g. Figure $3.4 \mathrm{p}$ ) and changes of walking speed (e.g. Figure 3.4 d). Although Figure 3.4 only shows the results for trailing limb angle, similar outcomes were found for other subtasks of gait.

\subsection{Discussion and conclusion}

We described a new controller that can automatically adapt the assistance that a robotic gait trainer applies based on users' performance during walking. A major advantage of this AT controller is that it can adjust the required assistance 
autonomously and independently per subtask of gait. In addition to this, it has the potential to result in challenging gait training because the assistance changes within a session based on users' performance.

To determine the feasibility of our controller as an assessment tool, the effect of PBWS and walking speed on walking performance was tested in ten healthy participants who walked in LOPES II at various walking speeds and levels of PBWS. We found that users' gait performances (i.e. errors) were significantly affected by the amount of PBWS for all subtasks of gait when participants did not receive robotic assistance. This is in agreement with previous studies about changes in kinematics when PBWS was applied [9], 10]. However, these previous studies found less relevant changes in performance for PBWS lower than $50 \%$. A possible reason for this might be that participants in our study walked in LOPES II, while participants in other studies walked freely on a treadmill [10 or in the Lokomat gait trainer 9]. These devices could influence the walking pattern and changes at various PBWS levels in a different way.

Although reference gait patterns of LOPES II were adjusted based on walking speed [11], effects of speed on users' performances were also significant for two of the five subtasks (stability during stance and trailing limb angle). A possible reason for this is that reference trajectories for LOPES II were determined based on free treadmill walking (without exoskeleton) [11. As LOPES II is not perfectly transparent 2], the robot might have influenced the way that users adapted their walking patterns at specific walking speeds.

Our results indicated that the AT controller cannot directly be used as an assessment tool (e.g. to compare between patients) as the performances were dependent on the levels of PBWS and walking speed. Changes in performance and assistance during therapy might be falsely attributed to changes in patients' capacities whereas they are actually caused by changes in PBWS or walking speed. This means that we either need to control for PBWS and walking speed when making comparisons within patients, or we need to improve our reference trajectories including the dependencies on PBWS. However, in the latter aspect, it might be difficult to do this, as we already incorporated the speed dependencies and we still saw effects of speed in our results. Nevertheless, our AT controller could be used as a tool to monitor progress during therapy, at constant PBWS and walking speed, and to improve RAGT by helping therapists to tailor the training to the capacities of a specific patient.

Experiments with the AT controller showed that the controller gave good responses and robotic assistance was adapted if needed. As expected, most participants did not receive much robotic assistance, as their performances were larger than the selected threshold. Further experiments in people with neurological disorders are needed to evaluate the performance of the controller. In future studies, the adaptive controller will be tested in people with neurological disorders to get more insight into its capacities as a monitoring tool during therapy, and as a therapeutic tool after neurological disorders. 


\section{References}

[1] T. Aurich-Schuler, F. Grob, H. J. Van Hedel, and R. Labruyère, "Can Lokomat therapy with children and adolescents be improved? An adaptive clinical pilot trial comparing Guidance force, Path control, and FreeD", Journal of NeuroEngineering and Rehabilitation, vol. 14, no. 1, pp. 1-14, 2017, ISSN: 17430003. DOI: 10.1186/s12984-017-0287-1.

[2] J. Meuleman, E. H. van Asseldonk, G. van Oort, H. Rietman, and H. van der Kooij, "LOPES II - Design and Evaluation of an Admittance Controlled Gait Training Robot with Shadow-Leg Approach", IEEE Transactions on Neural Systems and Rehabilitation Engineering, vol. 24, no. 3, pp. 352-363, 2016, ISSN: 15344320. DOI: 10.1109/TNSRE.2015.2511448

[3] C. Bayón, O. Ramírez, J. Serrano, M. D. Castillo, A. Pérez-Somarriba, J. Belda-Lois, I. Martínez-Caballero, S. Lerma-Lara, C. Cifuentes, A. Frizera, and E. Rocon, "Development and evaluation of a novel robotic platform for gait rehabilitation in patients with Cerebral Palsy: CPWalker", Robotics and Autonomous Systems, vol. 91, pp. 101-114, 2017, ISSN: 09218890. DOI: $10.1016 /$ j.robot .2016 .12 .015 .

[4] V. Bartenbach, M. Gort, and R. Riener, "Concept and design of a modular lower limb exoskeleton", in Proceedings of the IEEE RAS and EMBS International Conference on Biomedical Robotics and Biomechatronics, vol. 2016-July, 2016, pp. 649-654, ISBN: 9781509032877. DOI: 10 . 1109 / BIOROB.2016.7523699.

[5] S. Lefmann, R. Russo, and S. Hillier, The effectiveness of robotic-assisted gait training for paediatric gait disorders: Systematic review, 2017. DOI: 10.1186/s12984-016-0214-x.

[6] J. Cao, S. Q. Xie, R. Das, and G. L. Zhu, "Control strategies for effective robot assisted gait rehabilitation: the state of art and future prospects.", Medical engineering 85 physics, vol. 36, no. 12, pp. 1555-66, 2014, ISSN: 1873-4030. DOI: $10.1016 / \mathrm{j}$.medengphy.2014.08.005

[7] S. Maggioni, A. Melendez-Calderon, E. H. F. van Asseldonk, V. KlamrothMarganska, L. Lünenburger, R. Riener, and H. van der Kooij, Robot-aided assessment of lower extremity functions: A review, 2016. DOI: 10.1186/ s12984-016-0180-3.

[8] S. Maggioni, L. Lünenburger, R. Riener, and A. Melendez-Calderon, "Robot-Aided assessment of walking function based on an adaptive algorithm", IEEE International Conference on Rehabilitation Robotics, pp. 804-809, 2015, ISSN: 19457901. DOI: 10.1109/ICORR.2015.7281301.

[9] K. Van Kammen, A. M. Boonstra, L. H. Van Der Woude, H. A. ReindersMesselink, and R. Den Otter, "The combined effects of guidance force, bodyweight support and gait speed on muscle activity during able-bodied walking in the Lokomat", Clinical Biomechanics, vol. 36, pp. 65-73, 2016, ISSN: 18791271 . DOI: $10.1016 / \mathrm{j}$.clinbiomech.2016.04.013. 
[10] H. J. A. van Hedel, L. Tomatis, and R. Müller, "Modulation of leg muscle activity and gait kinematics by walking speed and bodyweight unloading", Gait and Posture, vol. 24, no. 1, pp. 35-45, 2006, ISSN: 09666362. DOI: 10.1016/j.gaitpost.2005.06.015.

[11] B. Koopman, E. H. F. van Asseldonk, and H. van der Kooij, "Speeddependent reference joint trajectory generation for robotic gait support", Journal of Biomechanics, vol. 47, no. 6, pp. 1447-1458, 2014, ISSN: 18732380. DOI: $10.1016 / \mathrm{j} . \mathrm{jbiomech} .2014 .01 .037$. 


\title{
Chapter 4
}

\section{Automatic versus manual tuning of robot-assisted gait training in people with neurological disorders}

\author{
S.S. Fricke, C. Bayón, H. van der Kooij, E.H.F. van Asseldonk \\ This chapter was published in Journal of NeuroEngineering and Rehabilitation, \\ 2020, 17:9.
}

\begin{abstract}
In clinical practice, therapists choose the amount of assistance for robot-assisted training. This can result in outcomes that are influenced by subjective decisions, and tuning of training parameters can be time-consuming. Therefore, various algorithms to automatically tune the assistance have been developed. However, the assistance applied by these algorithms has not been directly compared to manuallytuned assistance yet. In this study, we focused on subtask-based assistance and compared automatically-tuned (AT) robotic assistance with manually-tuned (MT) robotic assistance.

Ten people with neurological disorders (six stroke, four spinal cord injury) walked in the LOPES II gait trainer with AT and MT assistance. In both cases, assistance was adjusted separately for various subtasks of walking (in this study defined as control of: weight shift, lateral foot placement, trailing and leading limb angle, prepositioning, stability during stance, foot clearance). For the MT approach, robotic assistance was tuned by an experienced therapist and for the AT approach an algorithm that adjusted the assistance based on performances for the different subtasks was used. Time needed to tune the assistance, assistance levels and deviations from reference trajectories were compared between both approaches. In addition, participants evaluated safety, comfort, effect and amount of assistance for the AT and MT approach.

For the AT algorithm, stable assistance levels were reached quicker than for the MT approach. Considerable differences in the assistance per subtask provided by the two approaches were found. The amount of assistance was more often higher
\end{abstract}


for the MT approach than for the AT approach. Despite this, the largest deviations from the reference trajectories were found for the MT algorithm. Participants did not clearly prefer one approach over the other regarding safety, comfort, effect and amount of assistance.

Automatic tuning had the following advantages compared to manual tuning: quicker tuning of the assistance, lower assistance levels, separate tuning of each subtask and good performance for all subtasks. Future clinical trials need to show whether these apparent advantages result in better clinical outcomes.

\subsection{Introduction}

Robot-assisted gait training (RAGT) is a promising technique for rehabilitation after neurological disorders such as stroke or spinal cord injury (SCI). RAGT can be used to provide intensive, repetitive and task-specific training, while it also contributes to reduce physical load for therapists [1]. Reviews of previous studies have shown that RAGT can increase the likelihood that people walk independently after stroke, and that it is most effective in the acute phase after stroke/SCI and in the most impaired patients [2], 3. However, those results should be handled with some care as differences in patient groups, robotic gait trainers, protocol guidelines and control algorithms can largely affect the outcomes [2], 4].

Regarding protocol guidelines and control algorithms, it has to be considered that the amount of assistance that the robotic gait trainers provide to the users is often manually tuned by therapists or cannot be changed [5]-7]. Therapists mainly base their decisions on visual assessments of the patient, which means that training outcomes can be influenced by subjective decisions. Some studies address this issue by defining guidelines on how to set the assistance 6]- 9. However, these guidelines are often not really specific and require experienced therapists to adjust training parameters.

Therapists might have difficulties while tuning the assistance for RAGT compared to manually assisted gait training (where therapists use their hands to move patient's legs) due to two main reasons. First, in RAGT, therapists cannot directly feel the assistance that is being applied, and have to rely on other feedback (e.g. visual assessment of the patient) to choose the best assistance for the patient's needs. Second, the large number of parameters to tune the provided amount/timing of assistance [10], makes it difficult and time-consuming to manually change them while observing the patient [4]. Therefore, manually-tuned controllers that are currently used for therapy have their limitations in tailoring therapy to the patients' needs.

To objectively and quickly tune the robotic assistance and to promote active participation of the patient, various algorithms that automatically adjust the amount of robotic assistance for lower limbs [1] -21 or upper limbs 22 -26 , have been developed. Some of these algorithms gradually adapt the assistance based on an error compared to a reference trajectory and a forgetting factor [13, [14, [16, 21]. Others use reference trajectories (e.g. for the hip and knee angle during walking) with an (adaptive) virtual tunnel around these trajectories [11], [12, 25. Forces are applied by the device to prevent that the user moves out of the tunnel (i.e. too large deviations of joint angles compared to the reference trajec- 
tories). Most of these algorithms can tune the robotic assistance automatically and quickly at a joint level for each percentage of the gait cycle. However, they do not explicitly consider the different subtasks of walking (in this study defined as control of: weight shift, lateral foot placement, trailing and leading limb angle, prepositioning, stability during stance, foot clearance) [10], 27]-30].

We previously developed an algorithm that is focused on these functional subtasks of gait and automatically tunes the amount of robotic assistance for each subtask based on the user's performance during walking [15, [31. This algorithm is designed to tune the assistance in a similar way as therapists would like to tune robotic assistance: judging which subtasks of gait are affected and applying assistance for these subtasks 32 .

So far, automatically-tuned (AT) algorithms have mainly been evaluated in single sessions (e.g. effect on kinematics or EMG) [12, [16] or studies with a low number of participants [11], 33] while various larger clinical studies compared manually-tuned (MT) RAGT to conventional physical therapy 2]. As far as we know, the amount of robotic assistance applied by an AT algorithm has not been compared yet to the settings that a therapist would use and it is unknown how these two approaches affect rehabilitation in people with neurological disorders.

In the present, exploratory study, as a first step in getting more insight into the effect of MT and AT robotic assistance, we compare two different approaches for tuning robotic assistance by using the LOPES II gait trainer [10]: 1. subtaskbased assistance set by an experienced therapist (manually-tuned, MT); and 2 . subtask-based assistance set by our above-mentioned algorithm (automaticallytuned, AT) [15], 31]. By performing this comparison, we expect to answer the following questions: 1. How is the assistance tuned by the MT and AT approaches? (e.g. how long does it take to tune the assistance?); 2. Which final assistance levels are chosen for the MT and AT approach?; 3. How do these assistance levels affect deviations from the reference trajectories at specific evaluation points for each subtask (e.g. maximal hip and knee flexion)?; 4. Do the participants prefer one of the approaches over the other one regarding safety, comfort, effect and amount assistance?

The results from this study give more insight into how the two approaches, AT and MT assistance, affect RAGT and may be used to further optimize robot-based rehabilitation of patients with neurological disorders.

\subsection{Methods}

\subsubsection{Participants}

Six stroke survivors and four people with incomplete SCI, all in the chronic phase ( $>6$ months after injury), participated in this study ( 7 male, age $53 \pm 17$ years, weight $78 \pm 12 \mathrm{~kg}$, height $1.76 \pm 0.12 \mathrm{~m}$ ). An overview of the participants' characteristics can be found in Table 4.1. Inclusion criteria used in this study were 1 . age $>18$ years, 2 . a stable medical condition, 3 . a physical condition which allowed for 3 minutes of supported walking, 4 . sufficient cognitive abilities to follow the instructions and report any discomfort, 5. time since stroke/SCI $>6$ months. People with other orthopedic or neurological disorders or cardiac conditions that 
could be affected by physical load were excluded.

The experiments were approved by the local medical ethical committee (METC Twente) in accordance with the guidelines of the Declaration of Helsinki. All participants received oral and written information about the experiments and gave written informed consent prior to the start of the experiments.

\subsubsection{Robotic gait trainer}

LOPES II (LOwer extremity Powered ExoSkeleton II) was used to evaluate the AT and MT approach in this study. LOPES II is a gait trainer consisting of push-pull rods which are attached to the pelvis and lower limbs of the user [10. LOPES II can provide assistance for eight degrees of freedom (DOFs) (pelvis front/back, pelvis left/right, hip flexion/extension, hip abduction/adduction and knee flexion/extension) while the user is walking on an instrumented treadmill. LOPES II is an admittance-controlled device and the amount of robotic assistance can be set from minimal impedance (transparent mode, minimizing interaction forces between the device and human) to full assistance (mimicking position control). When applying assistance, LOPES II can move the user along different reference trajectories. The reference trajectories are defined for each DOF and are based on a data set from healthy elderly subjects [34]. The exact amount of force/torque that is applied to move the user along the reference trajectories depends on: 1 . deviations from the reference trajectories and 2. stiffness $K$ of virtual springs with equilibrium positions on the reference trajectories. This virtual spring stiffness $K$ can be calculated with the following equation for each DOF $(j)$ and each instant $(i$ in \%) of the gait cycle: $K_{j, i}=K_{\max , j}\left(\frac{G_{j, i}}{100}\right)^{2} . K_{\max , j}$ is a maximal stiffness that is defined for each DOF of LOPES II (see [10]) and $G_{j, i}$ is the desired assistance that is either MT or AT in this study. More details about the design and control of LOPES II can be found in 10].

\section{Subtask-based assistance}

The gait cycle was divided into various subtasks that are relevant for walking 10. (see Table 4.2 for an overview of the subtasks). Specific assistance profiles were used to assist when needed only at the portion of the gait cycle corresponding to each specific subtask (see Table 4.2). The subtask-based assistance could be adjusted individually, and separately for each leg. For example, left hip flexion could be assisted during swing to improve the leading limb angle on that side, while all other subtasks were in minimal impedance mode. As previously indicated, the assistance for each subtask was either chosen by a therapist (MT) or automatically calculated by the algorithm described below.

\section{Manually-tuned (MT) assistance}

A graphical user interface (GUI) was used by an experienced physical therapist to set the amount of robotic assistance [10] (see Figure 4.1). The GUI consisted of one main tab and one tab for each subtask. The subtask-based assistance could be adjusted individually with a minimal change of $10 \%$ by using a slider in the 
Table 4.1: Overview of participant characteristics, clinical scores and settings for LOPES II. ID: identification code used for each specific participant, 10MWT: 10 meter walking test, FAC: functional ambulation scale, FMA: Fugl-Meyer assessment (lower extremity), MI: Motricity index (lower extremity), AFO: ankle foot orthosis, Str..: participants with stroke, SCI..: participants with SCI.

\begin{tabular}{|c|c|c|c|c|c|c|c|c|c|c|c|c|}
\hline ID & Gender & Age & $\begin{array}{l}\text { Time } \\
\text { post } \\
\text { stroke/S } \\
\text { (years) }\end{array}$ & $\begin{array}{l}\text { More } \\
\text { af- } \\
\text { Ifected } \\
\text { leg }\end{array}$ & $\begin{array}{l}\text { Level } \\
\text { SCI }\end{array}$ & $\begin{array}{l}\text { 10MWT } \\
(\mathrm{km} / \mathrm{h})\end{array}$ & $\begin{array}{l}\text { Assistive } \\
\text { device(s) } \\
\text { used during } \\
\text { 10MWT }\end{array}$ & FAC & $\mathrm{FM}$ & $\mathrm{MI}$ & $\begin{array}{l}\text { Walking } \\
\text { speed in } \\
\text { LOPES } \\
\text { II }(\mathrm{km} / \mathrm{h})\end{array}$ & $\begin{array}{l}\text { Toe- } \\
\text { lifter in } \\
\text { LOPES } \\
\text { II }\end{array}$ \\
\hline Str1 & $\mathrm{m}$ & 53 & 1 & $\mathrm{r}$ & n.a. & 1.9 & cane, AFO & 4 & 13 & 28 & 1.5 & yes \\
\hline Str2 & $\mathrm{f}$ & 63 & 5 & 1 & n.a. & 2.1 & cane & 5 & 33 & 83 & 2.0 & no \\
\hline Str3 & $\mathrm{m}$ & 60 & 2 & 1 & n.a. & 2.4 & cane, AFO & 5 & 19 & 42 & 1.5 & yes \\
\hline Str4 & $\mathrm{m}$ & 33 & 10 & $\mathrm{r}$ & n.a. & 3.1 & none & 5 & 25 & 67 & 1.4 & no \\
\hline Str5 & $\mathrm{m}$ & 74 & 4 & $\mathrm{r}$ & n.a. & 4.1 & none & 5 & 31 & 91 & 1.7 & no \\
\hline Str6 & f & 74 & 6 & $\mathrm{r}$ & n.a. & 3.0 & none & 5 & 24 & 58 & 1.5 & no \\
\hline SCI1 & $\mathrm{f}$ & 45 & 8 & l & T9 & 2.8 & walker & 5 & n.a. & n.a. & 1.3 & no \\
\hline SCI2 & $\mathrm{m}$ & 25 & 3 & 1 & L3 & n.a. & n.a. & 0 & n.a. & n.a. & 1.5 & yes \\
\hline SCI3 & $\mathrm{m}$ & 63 & 6 & $\mathrm{r}$ & T12 & 0.8 & walker, AFO & 3 & n.a. & n.a. & 1.0 & yes \\
\hline SCI4 & $\mathrm{m}$ & 41 & 1 & $\mathrm{r}$ & L3 & 2.9 & none & 5 & n.a. & n.a. & 2.0 & no \\
\hline
\end{tabular}


Table 4.2: Overview of subtasks. Reference (black dotted lines) and measured (orange lines) positions and joint angles ( $P_{r e f}, P_{r e f}$, $\vartheta_{r e f}$, $\left.\vartheta_{\text {ref }}\right)$, assistance profiles $(K)$ and evaluation points (e.g. $\left.P_{\text {ref,e1 }}\right)$ that were used to calculate the error are shown. Each figure shows one gait cycle starting with left heel strike at 0\%. Weight shift to the right side, abduction and flexion angles are defined positive. The lower and upper bound are thresholds for adjusting the assistance based on the calculated error with the AT algorithm. If the error was lower than the lower bound, assistance was decreased. An error larger than the upper bound led to an increase in assistance and in other cases the assistance remained constant (see also Figure 4.1).

\begin{tabular}{llll}
$\begin{array}{l}\text { Subtask (affected DOF: } \\
\text { evaluation point(s)) }\end{array}$ & $\begin{array}{l}\text { Reference and } \\
\text { measured } \\
\text { trajectories, } \\
\text { evaluation points }\end{array}$ & $\begin{array}{l}\text { Stiffness profile, } \\
\text { reference } \\
\text { trajectory }\end{array}$ & Calculation of error \\
\hline $\begin{array}{l}\text { Weight shift (lateral pelvis } \\
\text { position: minimal and maximal } \\
\text { position) }\end{array}$ & $\begin{array}{l}\text { Lower } \\
\text { and upper } \\
\text { bound }\end{array}$ \\
$\begin{array}{l}\text { Lateral foot placement (hip } \\
\text { of gait cycle) }\end{array}$ & $\begin{array}{l}\text { Leading limb angle (hip } \\
\text { flexion angle: maximal angle) }\end{array}$
\end{tabular}


Prepositioning (knee flexion angle: angle at $100 \%$ of gait cycle)

Stability during stance (knee flexion angle: maximal angle between 10 and $40 \%$ of gait cycle)

Foot clearance (knee flexion angle: maximal angle)

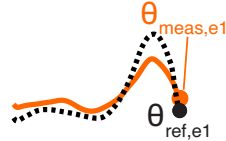

$\theta_{\text {refe }}$

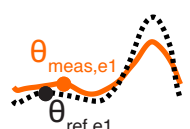

$\sqrt{1 . . . . . . . . . ~}$

$\theta_{\text {err }}=\left|\theta_{\text {ref }, e 1}-\theta_{\text {meas }, e 1}\right|$

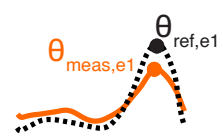

4.30deg

7.16deg.

$4.29 \mathrm{deg}$.

7.16deg.

4.52deg.

$7.54 \mathrm{deg}$. 
respective tab of the GUI. In addition, assistance levels for (all) subtasks could be coupled and the assistance levels for all coupled subtasks could be changed simultaneously by using a slider in the main tab of the GUI. To assist in tuning and show the immediate effects of changing assistance levels, visual feedback about the performance was provided for each subtask in the respective tab of the GUI (e.g. maximal knee flexion was shown for the foot clearance subtask, see Figure 4.1. In this study, one therapist, who was experienced in using LOPES II, tuned the amount of assistance for all experiments. The therapist got the instruction to set the assistance to a level that he would have used to train the patient. We decided not to give him more specific instructions as we were interested in which levels a therapist would choose without receiving any additional instructions.

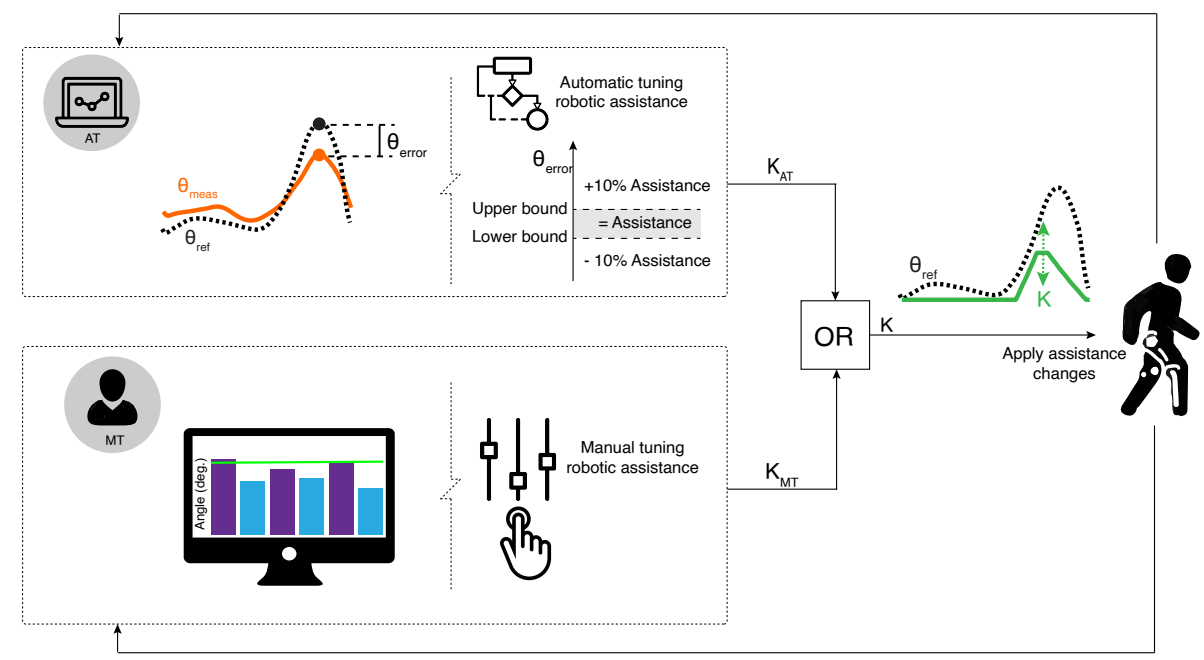

Figure 4.1: Overview of assistance tuning. The assistance was either AT based on the error between reference and measured trajectories or MT by a therapist. In this figure only an example for the foot clearance subtask is shown, however, the algorithm was applied to all subtasks shown in Table 4.2 simultaneously. For the AT algorithm, based on the error, every three steps, the assistance was either increased (if error $>$ upper bound, see Table 4.2), decreased (if error<lower bound) or kept constant (other cases) by scaling the amplitude of the assistance profile (K) shown on the right. For the MT approach, the therapist could change the assistance (amplitude of the assistance profile $K$ on the right) for each subtask by using graphical sliders. Feedback for the therapist was also shown to assist the therapist in tuning the assistance. As shown in this figure, the therapist got feedback about the maximal knee angle for the foot clearance subtask. The purple bars represented the maximal knee flexion angles for the previous three steps of the less impaired leg, while the blue bars represented the maximum knee flexion angles for the more impaired leg. The green line indicated the maximal knee flexion angle for the reference trajectory. 


\section{Automatically-tuned (AT) assistance}

The AT algorithm adjusted the amount of assistance based on the user's performance [15], 31] (see Figure 4.1). Specific evaluation points were defined for each subtask of walking (see Table 4.2). The reference and measured joint angles were determined for each evaluation point and the error was calculated as defined in Table 4.2. For some subtasks (foot clearance, trailing and leading limb angle, prepositioning), we assumed that exceeding the reference trajectory would not be detrimental. For example, we allowed maximum knee flexion larger than the reference gait pattern for the foot clearance subtask as too much knee flexion during swing is not typically found in people with stroke or SCI. In addition, the reference trajectories that were used in LOPES II are based on average trajectories of healthy individuals and might not exactly fit the needs of the user (with stroke or SCI). Allowing more knee flexion during swing (more foot clearance) than the reference pattern is safer as the feet will less likely hit the ground prematurely in the swing phase. For the same subtask, a knee flexion smaller than the reference pattern was penalized. For other subtasks (weight shift, stability during stance, lateral foot placement), we calculated the absolute error since an error in both directions might have negative consequences in people with neurological disorders. For example, during stance phase (subtask: stability during stance), both, knee hyperextension or too much knee flexion, can be found in people with neurological disorders 35.

Lower and upper bounds were defined for the subtask-based assistance based on the variability in the evaluation points in healthy participants walking in LOPES II in minimal impedance mode (see Table 4.2 [15. After three steps, the average error per subtask and side was calculated to adjust the amount of robotic assistance for each subtask and side separately. The subtask-based assistance was increased by $10 \%$ if the average error was larger than the upper bound (see Figure 4.1), as the user needed more assistance to stay closer to the reference trajectory. If the average error was lower than the lower bound, the amount of assistance was decreased by $10 \%$ to prevent that the user only relied on the assistance and to promote active participation. If the error was in between the lower and upper bound, the robotic assistance was kept constant.

\subsubsection{Experimental procedures}

Each participant took part in two sessions (familiarization and experimental session) on two different days. The familiarization session was used to gather information about the participants (e.g. clinical scores) and practice walking in LOPES II. The experiments to compare AT and MT assistance were performed in the experimental session.

In the familiarization session, clinical tests (10 meter walking test (10MWT), Functional Ambulation Category (FAC), Fugl-Meyer assessment (FMA), Motricity index (MI)) were administered by a therapist. After this, participants' upper and lower leg lengths and pelvis width was measured and adjusted in the software and hardware settings of LOPES II. Participants were strapped into LOPES II and toe-lifters were attached if participants dragged their toes along the ground 
during the swing phase. Participants with stroke, if needed, only used a toe-lifter on the more impaired side while participants with SCI used toe-lifters for both feet. Walking speed and, if needed, partial body weight support (PBWS) was set to a comfortable value based on the feedback from the participant and therapist (see Table 4.1). To get used to walking in LOPES II, participants walked at least two times, for three minutes in the device in this familiarization session. The first time, the assistance was set manually while the second time the AT algorithm was used to allow the user to experience both approaches. Participants were allowed to use the handrails of LOPES II during both sessions.

In the experimental session, the same settings (walking speed, PBWS, toelifters) as in the familiarization session were used to assess the AT and MT approach. Each participant performed four trials: $\mathrm{MT}_{\mathrm{var}}, \mathrm{MT}_{\text {const }}, \mathrm{AT}_{\mathrm{var}}$ and $\mathrm{AT}_{\text {const }}$ (var: variable assistance during the trial, const: constant assistance, as described below and in Table 4.3). Half of the participants started with MT assistance $\left(\mathrm{MT}_{\mathrm{var}}, \mathrm{MT}_{\text {const }}\right)$ and the other half started with $\mathrm{AT}$ assistance $\left(\mathrm{AT}_{\mathrm{var}}\right.$, $\left.\mathrm{AT}_{\text {const }}\right)$. Between the different trials, participants could take breaks. If needed, a break could be taken during $\mathrm{MT}_{\text {var }}$. If $\mathrm{AT}_{\text {const }}$ or $\mathrm{MT}_{\text {const }}$ was getting too exhausting for the participants, they could stop after less than three minutes. For both approaches, participants with a FAC score larger than 3, started at $30 \%$ of robotic assistance (following our clinical partner's advise), all other participants started at $100 \%$ assistance for all subtasks. The therapist was aware of the goal of the study and knew the goal of the AT controller. However, he was not present during the $\mathrm{AT}_{\text {var }}$ and $\mathrm{AT}_{\text {const }}$ trials to minimize bias.

In $\mathrm{MT}_{\mathrm{var}}$, the therapist set the amount of assistance using the GUI. While tuning the assistance, the therapist was able to visually assess the gait pattern and to get verbal feedback from the participant by talking to him/her. The therapist also received visual feedback about the performance for each subtask in the GUI. The therapist could take as much time as needed to set the robotic assistance to a final level that he/she would use for a training session with the specific participant. Subsequently, in $\mathrm{MT}_{\text {const }}$, the assistance was kept constant at the final assistance levels that the physical therapist had chosen in $\mathrm{MT}_{\mathrm{var}}$. Participants walked for three minutes with these settings.

Table 4.3: Overview of the trials of the experimental session. Each participant took part in all trials. Half of the participants started with the MT trials, while the other half started with the AT trials.

\begin{tabular}{lll}
\hline $\begin{array}{l}\text { Trial } \\
\text { name }\end{array}$ & Duration & Assistance \\
\hline $\mathrm{MT}_{\text {var }}$ & $\begin{array}{l}\text { as much as thera- } \\
\text { pist needed }\end{array}$ & $\begin{array}{l}\text { manually tuning assistance (therapist), vari- } \\
\text { able (var) assistance during the trial } \\
\text { constant (const) at level that therapist chose }\end{array}$ \\
$\mathrm{MT}_{\text {const }}$ & 3 minutes & $\begin{array}{l}\text { automatically tuning assistance, variable (var) } \\
\text { assistance during the trial } \\
\text { constant (const) at level of last } 15 \text { steps of } \\
\text { ATar }\end{array}$ \\
$\mathrm{AT}_{\text {const }}$ & 3 minutes & \\
\hline
\end{tabular}


In $\mathrm{AT}_{\mathrm{var}}$, participants walked for three minutes with the adaptive AT algorithm, which automatically adjusted the amount of robotic assistance based on users' performance as explained in the previous section. After three minutes, LOPES II was stopped. Subsequently, in $\mathrm{AT}_{\text {const }}$, participants walked for three minutes while keeping the subtask-based assistance constant at the average assistance levels calculated with the last 15 steps of $\mathrm{AT}_{\mathrm{var}}$ (rounded to the nearest tens).

\subsubsection{Outcome measures}

To analyze differences between the AT and MT approach, we focused on different aspects that are described in this section: assistance tuning, final amount of assistance, errors at final amount of assistance, PBWS and questionnaires that were filled in by the participants and the therapist.

\section{Assistance tuning}

The time at which a stable assistance level was reached, was determined for each participant and each subtask for $\mathrm{AT}_{\mathrm{var}}$ and $\mathrm{MT}_{\mathrm{var}}$. The AT algorithm might change the assistance by $10 \%$ every three steps, never reaching a completely stable level. Therefore, it was defined that a stable level was reached when no changes larger than 10\%, compared to the final assistance level of the trial, occurred. A two-sided Wilcoxon signed rank test was used to evaluate differences in the time that was needed to tune the assistance. A p-value lower than 0.05 was considered significant.

\section{Final assistance levels}

The applied robotic assistance was compared between $\mathrm{AT}_{\text {const }}$ and $\mathrm{MT}_{\text {const }}$ for each participant and each subtask of walking.

\section{Errors for final assistance levels}

The average error (difference between reference and measured trajectory) for $\mathrm{AT}_{\text {const }}$ and $\mathrm{MT}_{\text {const }}$ was calculated for each participant and subtask. In the results section we focus on the errors above the upper bounds (negative effects on participant's gait), which are defined in Table 4.2

\section{Partial body weight support}

Participants were allowed to use the handrails during walking and might have varied the amount of force applied to the handrails to support their own weight. To make sure that there were no large differences in the amount of PBWS between the MT and AT trials, the average PBWS was calculated by using the vertical forces measured with the force sensors under the walking surface of the treadmill. 


\section{Questionnaires}

Participants' preferences: Participants filled out a self-administered paperbased questionnaire about the trials with MT and AT assistance. The questionnaire contained the following four questions that were evaluated, for each approach, on a scale from 1 to 5 (1 being very unsatisfied and 5 being very satisfied):

How satisfied are you with ...

1. ...the safety experienced in the robot (do you feel safe)?

2. ...the comfort during walking in the robot (assistance or resistance)?

3. ...the effect of assistance on walking in the robot?

4. ...the amount of assistance given by the robot?

Average scores and standard deviations were calculated for each question that participants filled in.

Therapist: To get more insight into how the therapist was choosing the assistance provided by LOPES II, the therapist filled in a short questionnaire with the following two questions:

1. Which settings did you adjust and why?

2. Are you satisfied with the result? Why (not)? (For example, were there things that you could not change in the way you wanted?)

In this paper, only the most common answers are reported and we do not focus on specific answers that were only given for a small number of participants.

\subsection{Results}

All participants were able to perform the protocol and walk with the AT and MT algorithm. However, for SCI2, $\mathrm{AT}_{\text {const }}$ was stopped after two minutes (instead of three minutes) as the participant was getting too exhausted.

\subsubsection{Assistance tuning}

On average, a stable assistance level for $\mathrm{MT}_{\text {var }}$ (difference to final level $<10 \%$ for all subtasks) was reached after $279 \pm 120 \mathrm{sec}$. For $\mathrm{AT}_{\text {var }}$, a stable level was reached more quickly (after $110 \pm 54$ sec.). The Wilcoxon signed rank test indicated that this difference between the MT and AT approach was significant $(\mathrm{Z}=-3.60$, $\mathrm{p}=0.006)$.

For the AT approach, in the beginning of the trial, the assistance for each subtask was changed every three steps until it approached its final stable level (changes of maximal 10\%). In contrast to this, the therapist (MT approach) often focused on decreasing the assistance for all subtasks simultaneously (i.e. coupling all subtasks in the GUI) and then increasing the assistance for (one to four) specific subtasks. As an example, Figure 4.2 shows these differences in tuning the assistance for the hip and knee flexion of one participant (SCI3). 


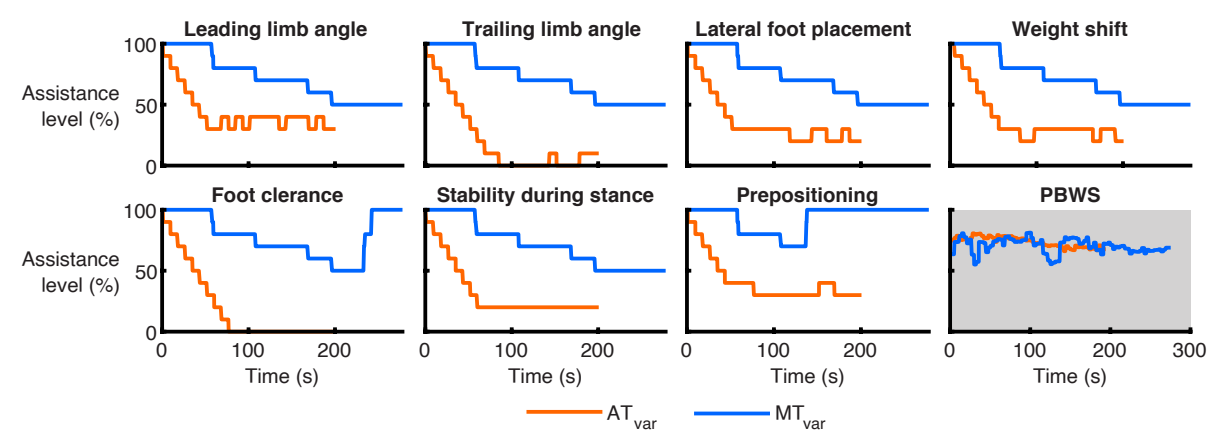

Figure 4.2: Assistance levels while tuning the assistance in SCI3. Assistance levels for all subtasks of the more impaired leg and weight shift are shown for $A T_{\text {var }}$ and $M T_{\text {var }}$ of participant SCI3. The subfigure with grey background shows the measured PBWS (provided by LOPES II and use of the handrails by the participant).

\subsubsection{Final assistance levels}

Large differences in the assistance levels that were applied in $\mathrm{AT}_{\text {const }}$ and $\mathrm{MT}_{\text {const }}$ were found for both legs and the weight shift subtask (see Figure 4.4 for the more impaired leg and Figure 4.6 for the less impaired leg). The weight shift subtask is shown in both figures (Figure 4.4 and 4.6 , grey background), however, it is considered separately in the text below.

Figure 4.3 shows an example of the differences in assistance levels and the resulting joint trajectories for the knee and hip joints of Str5.

\section{More impaired leg}

For both approaches, AT and MT assistance, a higher assistance was applied for up to 4 specific subtasks of the more impaired leg in each participant, while less (MT) or no (AT) assistance was applied for other subtasks (see Figure 4.4). Per participant, the therapist (MT approach) tuned 0 to 3 specific subtasks separately for the more impaired leg (see light blue bars in Figure 4.4) while all other subtasks were (simultaneously) set to the same assistance level (dark blue bars). In 12 of the 60 cases (the term 'cases' means subtasks for all participants (e.g. for the more impaired leg: 6 subtasks times 10 participants results in 60 cases)), the assistance for the more impaired leg was tuned separately by the therapist (see Table 4.4. In 11 of these 12 separately-tuned cases, the assistance was higher for $\mathrm{MT}_{\text {const }}$ compared to $\mathrm{AT}_{\text {const }}$ and for 1 of these 12 separately-tuned cases the same assistance was applied for both approaches. Also, for 33 of the 48 cases that were not tuned separately by the MT approach the assistance was higher in $\mathrm{MT}_{\text {const }}$ compared to $\mathrm{AT}_{\text {const }}$.

Remarkably, for $\mathrm{AT}_{\text {const }}$, the most impaired participants (SCI2 and SCI3) did not receive much assistance (max. 40\%) while these participants received at least $50 \%$ assistance for each subtask in $\mathrm{MT}_{\text {const }}$ (Figure 4.4). These participants could 


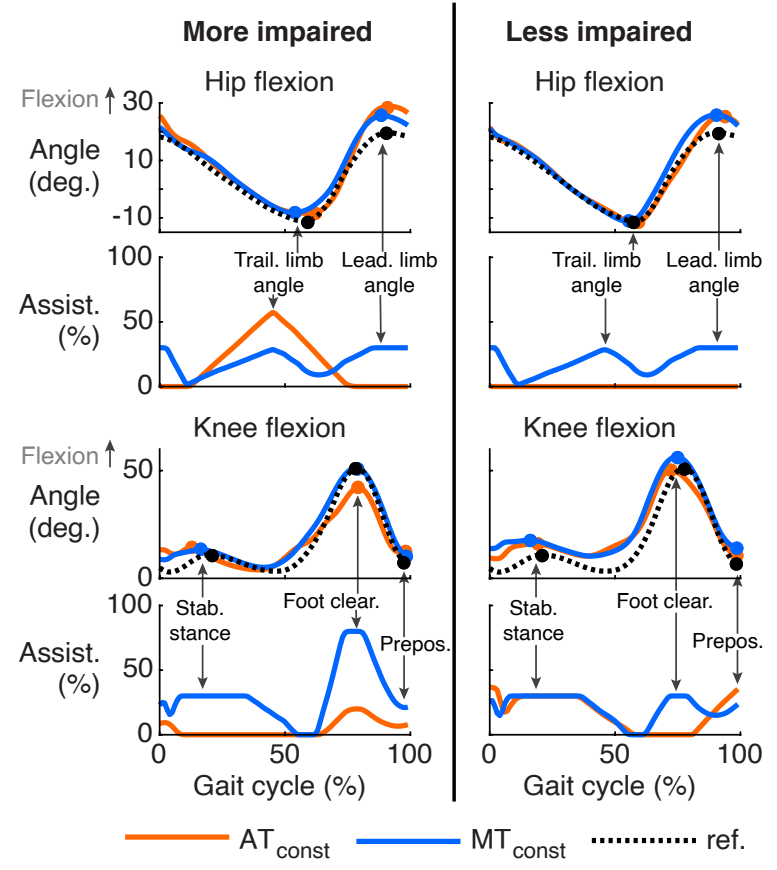

Figure 4.3: Average hip and knee flexion angles and assistance for Str5. Average angles and assistance across $A T_{\text {const }}$ and $M T_{\text {const }}$ are shown for Str5 for both legs as a function of gait cycle. The dots plotted on the trajectories indicate the evaluation points (see also Table 4.2) for the different subtasks.

probably walk with the low levels of assistance in $\mathrm{AT}_{\text {const }}$ due to the high levels of PBWS that were used (see Figure 4.5). Although the same PBWS levels were applied for $\mathrm{MT}_{\text {const }}$ for SCI2 and SCI3 (and other participants, see Figure 4.5p, considerable differences were found for the assistance levels (Figure 4.4). A possible reason for this is that the therapist was biased towards higher assistance levels due to the large impairments (i.e. low clinical scores) of SCI2 and SCI3 (Table 4.1). In addition, the therapist only knew the amount of PBWS provided by the system and he did not know the exact amount of PBWS as participants were using the hand rails for additional PBWS (see Figure 4.5).

\section{Less impaired leg}

For the less impaired leg, deviations from the reference trajectories were such that $\mathrm{AT}_{\text {const }}$ resulted in assistance for up to 3 specific subtasks in each participant while the remaining subtasks did not receive any assistance (see Figure 4.6). In contrast to this, with $\mathrm{MT}_{\text {const }}$ the assistance was not tuned separately in 58 of the 60 cases for the less impaired leg. For these 58 cases, the assistance applied by the MT approach was higher than the assistance applied by the AT approach in 34 cases (see Table 4.4). 

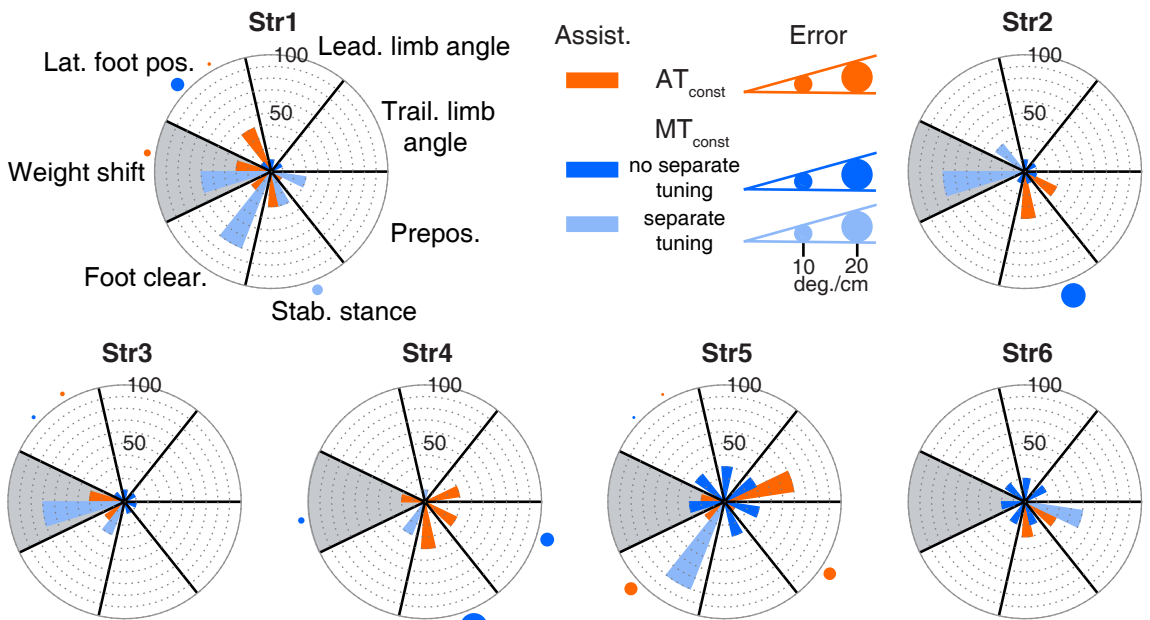

Str4
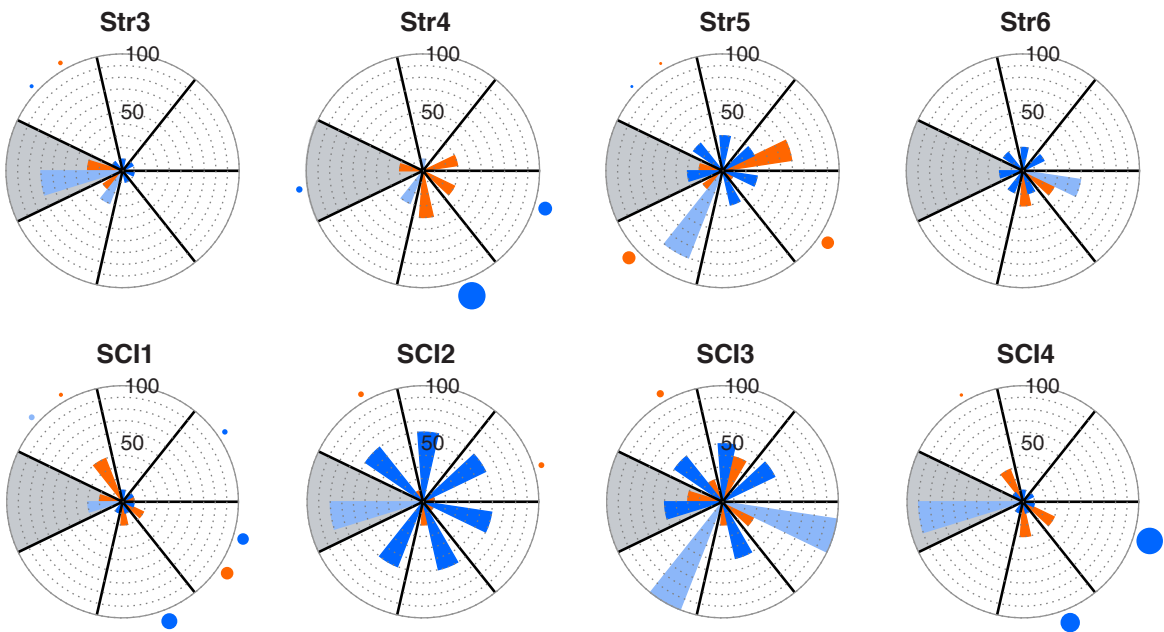

Figure 4.4: Assistance and errors for $A T_{\text {const }}$ and $M T_{\text {const }}$ for the more impaired leg (white background) and weight shift (grey background). Each polar plot shows the results of for participant. The distribution of the subtasks is the same for all polar plots (see Str1). The results for $M T_{\text {const }}$ are split up into subtasks that were separately tuned by the therapist in a specific participant (light blue) and subtasks that were not separately tuned (dark blue). The bars represent the amount of assistance that the participants received for each specific subtask. The circles outside of the polar plots represent the size of the error that was found for each specific subtask (see legend for scale). Only errors above the upper bound (as defined in Table 4.2) are shown.

\section{Weight shift}

The therapist (MT approach) separately changed the assistance for the weight shift in 6 of the 10 cases (see Figure 4.4 and 4.6, grey background). In all of these separately-tuned cases, the weight shift assistance was higher for the MT approach compared to the AT approach (see Table 4.4). Also, for the other 4 cases (no separate tuning of weight shift by the therapist), the assistance was higher for the MT approach in 3 cases. 
Table 4.4: Comparison of final assistance levels for MT and AT. Cases means subtasks for all participants (e.g. for the more impaired leg: 6 subtasks times 10 participants results in 60 cases). The cases are split up into cases that were tuned separately by the therapist in $M T_{\text {const }}$ and cases that were not separately tuned.

\begin{tabular}{llc}
\hline & Separate tuning (MT) & No separate tuning (MT) \\
\hline More impaired leg & $12 / 60$ cases & $48 / 60$ cases \\
MT $>$ AT assist. & $11 / 12$ & $33 / 48$ \\
MT=AT assist. & $1 / 12$ & $1 / 48$ \\
MT $<$ AT assist. & $0 / 12$ & $13 / 48$ \\
\hline Less impaired leg & $2 / 60$ cases & $58 / 60$ cases \\
MT $>$ AT assist. & $1 / 2$ & $34 / 58$ \\
MT=AT assist. & $0 / 2$ & $7 / 58$ \\
MT $<$ AT assist. & $1 / 2$ & $17 / 58$ \\
\hline Pelvis & $6 / 10$ cases & $4 / 10$ cases \\
MT $>$ AT assist. & $6 / 6$ & $3 / 4$ \\
MT $=$ AT assist. & $0 / 6$ & $0 / 4$ \\
MT $<$ AT assist. & $0 / 6$ & $1 / 4$ \\
\hline
\end{tabular}

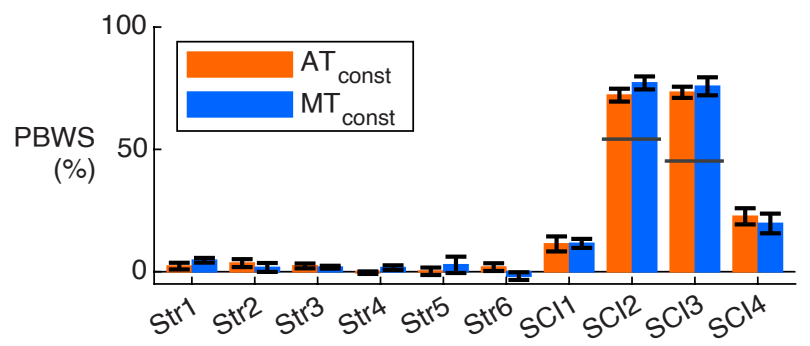

Figure 4.5: Partial body weight support. Average body weight support and standard deviation (between steps) for $A T_{\text {const }}$ and $M T_{\text {const. }}$. The bars show the total PBWS (from the system and the use of the handrails). Only SCI2 and SCI3 received PBWS from the system (55\% and 46\%, respectively, indicated by the horizontal grey lines). All other $P B W S$ is the result of using the handrails. Negative values can, for example, be explained by parts of LOPES II that might have slightly rested on the pelvis of the participant.

\subsubsection{Errors for final assistance levels}

For both legs and the weight shift subtask, differences in the amount and magnitude of errors above the upper bound, which is the error at which assistance would be increased by the adaptive AT algorithm (see Table 4.2), were found.

\section{More impaired leg}

For the more impaired leg and $\mathrm{MT}_{\text {const }}$, the error was larger than the upper bound in 2 of the 12 cases that were tuned separately by the therapist (MT approach, see light blue dots in Figure 4.4) and in 10 of the 48 cases that were not tuned separately (dark blue dots). For $\mathrm{AT}_{\text {const }}$, the error was larger than the upper 

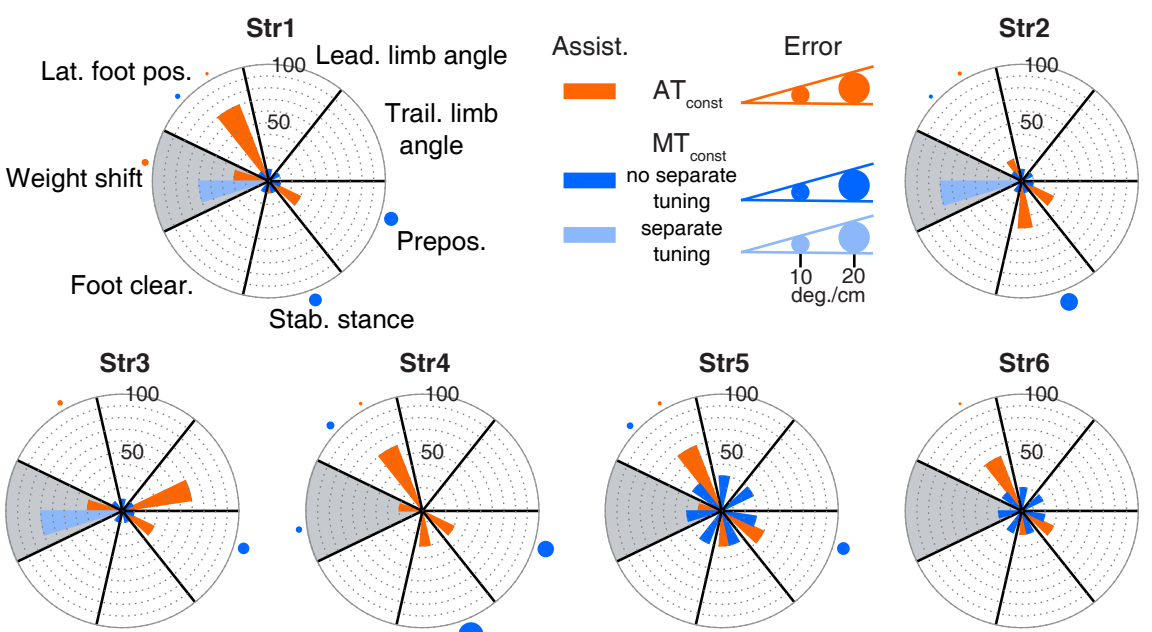

Str4
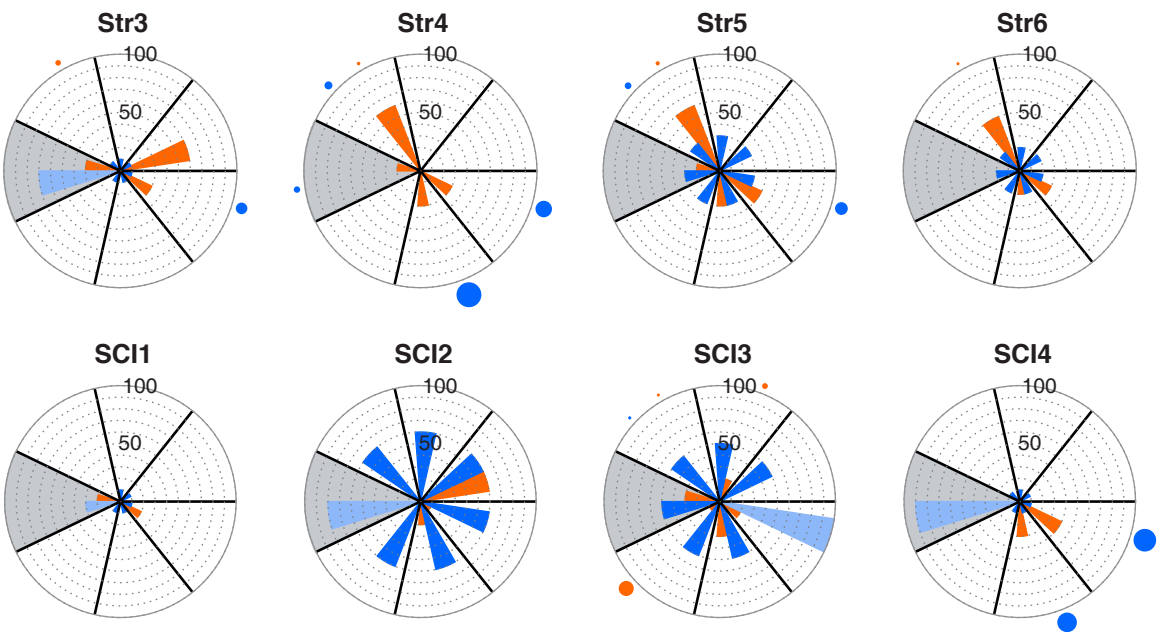

Figure 4.6: Assistance and errors for $A T_{\text {const }}$ and $M T_{\text {const }}$ for the less impaired leg (white background) and weight shift (grey background). Each polar plot shows the results for one participant. The distribution of the subtasks is the same for all polar plots (see Str1). The results for $M T_{\text {const }}$ are split up into subtasks that were separately tuned by the therapist in a specific participant (light blue) and subtasks that were not separately tuned (dark blue). The bars represent the amount of assistance that the participants received for each specific subtask. The circles outside of the polar plots represent the size of the error that was found for each specific subtask (see legend for scale). Only errors above the upper bound (as defined in Table 4.2) are shown.

bound in 10 of the 60 cases (orange dots). These errors for the AT algorithm were found because the algorithm did not adapt the assistance in $\mathrm{AT}_{\text {const }}$ and therefore, the assistance was not automatically increased when the error was larger than the upper bound.

Remarkably, although often less assistance was applied for $\mathrm{AT}_{\text {const }}$, the observed errors were much lower than for $\mathrm{MT}_{\text {const }}$ (always $<10$ deg.). The largest errors of up to $20 \mathrm{deg}$. of deviation from the reference trajectory were found for $\mathrm{MT}_{\text {const }}$, but only in subtasks that that were not separately tuned by the therapist (e.g. stability during stance subtask (e.g. Str2, Str4) and prepositioning (SCI4), see Figure 4.4. 


\section{Less impaired leg}

For the less impaired leg only two subtasks were tuned separately by the therapist and for these subtasks the error was lower than the upper bound. For $24 \%$ of the 58 subtasks that were not tuned separately, the error was larger than the upper bound (see dark blue dots in Figure 4.6. For $\mathrm{AT}_{\text {const }}$, the error was larger than the upper bound in only 9 of the 60 cases (orange dots).

The largest errors (up to $18 \mathrm{deg}$. of deviation from the reference trajectory) were found for $\mathrm{MT}_{\text {const }}$ for the stability during stance and prepositioning subtasks (see Figure 4.6). In most cases, the errors for $\mathrm{AT}_{\text {const }}$ were much lower. Only for one of the participants (SCI3) an error of $10 \mathrm{deg}$. was found for the foot clearance subtask in $\mathrm{AT}_{\text {const }}$, while all other errors were smaller than $10 \mathrm{deg}$.

\section{Weight shift}

Resulting errors for the weight shift subtask were generally small. Separate tuning of the weight shift subtask in $\mathrm{MT}_{\text {const }}$ always resulted in errors lower than the upper bound (see Figure 4.4 and 4.6). Only in one case the error was higher than the upper bound in $\mathrm{MT}_{\text {const }}$ when the assistance was not selectively tuned. The AT algorithm also resulted in errors lower than the upper bound in all except for one participants. The error was less than $5 \mathrm{~cm}$ in both cases $\left(\mathrm{MT}_{\text {const }}\right.$ and $\left.\mathrm{AT}_{\text {const }}\right)$.

\subsubsection{Questionnaires}

\section{Participants' preferences}

Participants evaluated safety, comfort and effect and amount of assistance on a scale with a maximum of 5 . On average, participants gave similar (high) scores for the safety (AT: 4.5, MT: 4.4) and the effect of assistance (AT: 4.0, MT: 3.9) (see Figure 4.7). The comfort was evaluated slightly better for the AT algorithm (4.0) compared to MT assistance (3.7). In contrast to this, participants were slightly more satisfied with the amount of assistance given by the MT algorithm (4.5) compared to the AT algorithm (4.1). The scores per participant were also checked to see whether there were clear differences between the two approaches in specific participants, however, the difference between AT and MT assistance was never larger than 1 for any of the questions.

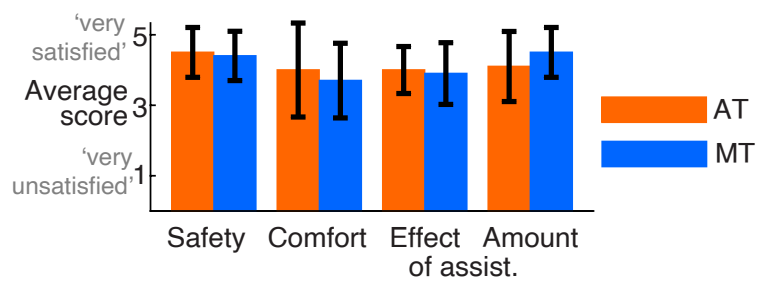

Figure 4.7: Average evaluation of the AT and $M T$ algorithms by all participants. The aspects safety, comfort, effect of assistance and amount of assistance were evaluated on a scale from 1 (very unsatisfied) to 5 (very satisfied). The average for all participants and standard deviation between participants is shown. 


\section{Therapist}

The therapist answered in eight of the ten participants that he/she adjusted the assistance for specific subtasks separately. The therapist decreased the assistance for all other subtasks to assist the most impaired subtasks, but let the participants do as much as possible by themselves. For four of the ten participants the therapist was satisfied with the result. For the other participants he/she was not satisfied with the exact effect of the assistance. Besides, the therapist claimed that it was often difficult to see what exactly changed (e.g. when decreasing the assistance), and that he/she sometimes had to rely on feedback from the participants.

\subsection{Discussion}

The goal of this study was to compare subtask-based MT and AT robotic assistance during gait in people with neurological disorders. We determined differences while tuning the assistance, final assistance levels, errors compared to reference trajectories and preferences of the participants. For all of these aspects, large differences were found between the AT and MT approach, except for the preferences of the participants, which were similar for both approaches.

\subsubsection{Possible reasons for differences between the AT and MT ap- proach}

There might be several reasons for the large differences in final assistance levels (and deviations from the reference trajectories) between the two approaches that can only be speculated on. The AT algorithm assured a good performance for all subtasks by tuning the assistance for each subtask separately. In contrast to this, the therapist (MT approach) tuned a small number of subtasks separately (the most affected ones) and aimed for a good performance (low errors) for these subtasks. For the subtasks that were not tuned separately, the largest errors were found, which means that the therapist accepted larger deviations for these subtasks. Although the therapist could have used the GUI to see the deviations from reference trajectories for all subtasks, he/she was mainly relying on visual assessment of the gait pattern and feedback from the participants when tuning the assistance. This could be an indication that the therapist did not attempt to decrease the deviations from the reference trajectories for all subtasks, but rather tried to reach an acceptable walking pattern. In addition, the therapist might have accepted larger deviations from reference trajectories to allow for compensation strategies.

Another possible reason for the differences between the AT and MT approach is that tuning all subtasks separately could be too complicated and time-consuming for clinical practice. The subtasks were related to common problems after neurological disorders $36-[40$. They were chosen based on input from physical therapists and rehabilitation physicians who indicated that they would like to have more possibilities to tune the assistance than in other (commercially available) robotic gait trainers, which often only allow to change the general assistance for the whole gait cycle and multiple joints simultaneously [4, 32]. The number of 
subtasks in the current study is relatively low (6 for each leg, and weight shift). Still, in this study, the therapist focused only on a low number of subtasks (up to 4 per participant) and tuned these subtasks separately.

A last possible reason for the difference between the AT and MT approach is that the therapist might also have acted on the safe side, by trying to prevent possible problems occurring with (too) low assistance levels (e.g. stumbling, exhaustion) and therefore more often higher assistance levels were found for the MT approach. An indication for this could be that for the most impaired participants the MT assistance was much higher than needed, even for most subtasks that were not tuned separately.

\subsubsection{Advantages of the AT approach compared to the MT approach}

A large advantage of the AT approach is that it is not influenced by subjective decisions of the therapist. However, there are various other factors that can be used to determine whether the AT or MT approach is better. In this study, we focused on the time to tune the assistance, the amount of assistance and deviations from reference trajectories.

The time to tune the assistance is an important factor that needs to be considered for clinical application. If the tuning takes too long, patients might not be able to exercise at their desired assistance levels as they might be too fatigued or the training session might end before the desired assistance levels are reached. In our study, the AT algorithm reached a constant assistance level more quickly than the MT algorithm. Two studies with other AT algorithms also have shown that stable assistance levels can be reached within a similar time as in our current study with an automatic algorithm [13, 21].

Another factor that we considered was the amount of assistance. From literature, it is known that active participation is an important factor in rehabilitation after neurological disorders and applying too much assistance might hinder recovery [4], 41]- 43]. There is accumulating evidence that focusing on algorithms that tailor therapy to the patient's needs by only applying as much assistance as needed, can increase training intensity and improve outcomes of RAGT. For example, Srivastava et al. 11] and Krishnan et al. 33] have shown that AT algorithms for RAGT can lead to improvements in clinical scales, however, no control groups were included in these studies to compare the AT algorithms to other approaches. Park et al. 44 found that progressively reducing the amount of assistance from $100 \%$ to $60 \%$ can lead to larger improvements in FAC score and Berg balance scale in people with subacute stroke compared to applying $100 \%$ assistance during a training program of four weeks. Though the evidence is still preliminary, these studies indicate that personalized and reduced robotic assistance leads to larger improvements. In this regard, better results were obtained for the AT algorithm in our current study: every subtask was tuned separately and the assistance was more often lower for the AT approach than for the MT approach.

Even though less assistance was often applied by the AT algorithm, the largest deviations from the reference trajectories were found for the MT approach. It is debatable how closely measured trajectories need to match reference trajectories 
(i.e. physiological trajectories) in RAGT as allowing compensatory mechanisms might also be beneficial [21], [42]. In the current study, the AT approach resulted in walking patterns close to the reference trajectories and assistance might have been increased to prevent compensatory strategies. In contrast to this, the therapist could have allowed compensatory strategies by decreasing assistance. In the future, the MT approach might be more suitable when compensatory strategies should be allowed, while the AT approach leads to smaller errors in the evaluation points (i.e. more physiological gait pattern).

Next to the factors that were analyzed in this study (time to tune the assistance, amount of assistance and deviations from reference trajectories), there are more factors that could influence the therapeutic effect of RAGT. For example, it is not known yet if assisting a specific subtask might lead to better clinical outcomes than assisting another specific subtask. In addition to this, applying less assistance might be more exhausting and result in shorter training duration (although fatigue might be partly compensated for by automatically increasing assistance with the AT algorithm). It is not known yet how shorter (but more intensive) robotic gait training sessions would affect therapy outcomes compared to longer (less intensive) training sessions [2].

To sum up, regarding the time to tune the assistance, the amount of assistance and deviations from reference trajectories, the AT algorithm has more advantages than the MT approach. However, we cannot draw any decisive conclusions about possible clinical outcomes yet as there are too many factors that might affect clinical outcomes.

\subsubsection{Study limitations}

Deriving reference trajectories for robot-assisted gait training is crucial but difficult. We used reference trajectories that depended on walking speed and body length 34 . However, these trajectories were collected during treadmill walking and did not take into account that the dynamics of the robot or PBWS could influence the gait pattern 15. It is still debated whether reference trajectories should be adjusted based on robot dynamics, PBWS or other therapeutic goals. In our current study, when using trajectories based on treadmill walking that were not adjusted to the specific gait trainer, maximal hip flexion was larger than the reference trajectory for nearly all participants (for the AT and MT approach). Therefore, the assistance that was applied for the leading limb angle subtask (mainly for the MT approach) might have impeded motion and decreased maximal hip flexion. Having the option to automatically (e.g. based on less impaired leg) or manually [10] change the reference trajectories might be useful for future training protocols.

Another limitation is that only one experienced therapist tuned the assistance in this study. For example, there might be differences in the settings that are applied by an experienced therapist compared to an inexperienced therapist (or compared to another experienced therapist). To our knowledge, there are no studies that compare the assistance that is applied by an experienced and inexperienced therapist for RAGT, especially not for LOPES II. Still, other studies analyzed differences between therapists for physical assistance that was applied 
during training. In [45], seven therapists applied similar forces to correct balance in stroke survivors during overground training. However, Galvez et al. [46] showed that the physical assistance applied to the legs of SCI patients during body weight supported treadmill training was different between experienced and inexperienced therapists. It is not clear yet what the exact reasons for the differences were and if they would also appear for tuning of RAGT. However, as various settings can be changed in LOPES II (six subtasks per leg, and weight shift) and the therapist in the current study was mainly relying on (subjective) visual assessment of the gait pattern, we would expect differences between therapists, especially between novice users and experienced therapists. Therefore, in future studies, experiments should be performed with multiple therapists (experienced and inexperienced) and/or therapists should be taught to rely more on the objective and quantitative feedback that is provided by the GUI as it is expected that this will lead to lower variability between therapists.

\subsubsection{Future directions}

Instead of choosing for either AT or MT assistance, in the future, a combined AT and MT approach might be used to take advantage of both approaches. Some possibilities that could be investigated in future studies are:

1. The AT algorithm could be used to give recommendations on the amount of assistance to apply while the therapist still has to take the final decision about which assistance levels are applied. The advantage of this is that the therapist's knowledge is taken into account, he/she has control over the training, he/she can take into account feedback from the patient and the AT algorithm might show that the user needs more assistance on certain subtasks that the therapist might not have taken into account otherwise. A disadvantage is that tuning of the assistance might be slower than with an AT algorithm alone.

2. The assistance for all subtasks is AT, however, the therapist could choose to tune some specific subtasks manually if he/she does not agree with the effect of the AT algorithm or wants to reduce specific errors even more. This would still give the therapist some control, the therapist could take into account feedback from the patient, but it would also make the whole process quicker as the therapist would not have to tune the exact assistance levels for each subtask anymore. Besides, compared to MT assistance alone which could be focused on a low number of subtasks, all subtasks would be tuned to the specific needs of the patient.

3. Another possibility would be that the therapist chooses more discrete levels (e.g. low, medium, high) which are each associated with a specific range of assistance levels (e.g. low from 0-30\%). Within these discrete levels an AT algorithm could choose the exact amount of assistance. In this case, the therapist would still be able to choose a broad assistance level based on his/her experience and feedback from the patient, and he/she is assisted by the AT algorithm in quickly choosing the exact level of assistance.

Although it is not known which combination would work best, we believe that a combination of AT and MT subtask-based assistance could be beneficial for future RAGT as it would take into account therapist's knowledge and experience, 
it allows the patient to give feedback, but it also simplifies tuning of the parameters compared to MT assistance alone.

In addition, it should be investigated whether the AT algorithm itself can be further improved. To promote active participation of the patient, our AT algorithm decreases the assistance when errors are small, however, it is not known yet whether adding a forgetting factor [14, [16] leads to even more active participation of the patient. It might also be beneficial to automatically tune other parameters (e.g. PBWS, walking speed) as these can also affect the gait pattern and amount of assistance that is applied by an AT algorithm [15].

\subsection{Conclusion}

We have found large differences in the assistance applied by an automaticallytuned and manually-tuned algorithm. Advantages of the AT approach compared to the MT approach were that the assistance was tuned quicker, lower assistance levels were used (enhancing active participation of the user), each subtask was tuned separately and a good performance was assured for all subtasks. In contrast to this, the MT approach focused on a limited number of subtasks (two to four) that were tuned separately. Future clinical trials need to show whether these apparent advantages of the AT approach result in better clinical outcomes. To exploit the advantages of the AT approach (e.g. quick tuning of all subtasks) and take into account the experience of therapists and feedback from patients during the training, a combined approach of manual and automatic tuning should be considered in the future.

The results from this study can be used to develop more extended (clinical) studies that are needed to get insight into the long-term effect of AT and MT subtask-based training protocols on walking function after neurological disorders.

\section{References}

[1] M. R. Spiess, F. Steenbrink, and A. Esquenazi, "Getting the Best Out of Advanced Rehabilitation Technology for the Lower Limbs: Minding Motor Learning Principles", Innovations Influencing Physical Medicine and Rehabilitation, vol. 10, no. 9, S165-S173, 2018, ISSN: 19341482. DOI: 10.1016/ j.pmrj.2018.06.007

[2] J. Mehrholz, S. Thomas, C. Werner, J. Kugler, M. Pohl, and B. Elsner, "Electromechanical-assisted training for walking after stroke (Review)", Cochrane Database of Systematic Reviews, no. 5, 2017. DoI: $10.1002 /$ 14651858. CD006185.pub4.

[3] K. Y. Nam, H. J. Kim, B. S. Kwon, J.-W. Park, H. J. Lee, and A. Yoo, "Robot-assisted gait training (Lokomat) improves walking function and activity in people with spinal cord injury: a systematic review", Journal of NeuroEngineering and Rehabilitation, vol. 14, no. 24, 2017, ISSN: 1743-0003. DOI: $10.1186 / \mathrm{s} 12984-017-0232-3$. 
[4] S. F. Atashzar, M. Shahbazi, and R. V. Patel, "Haptics-enabled Interactive NeuroRehabilitation Mechatronics: Classification, Functionality, Challenges and Ongoing Research", Mechatronics, vol. 57, pp. 1-19, 2019, ISSN: 09574158. DOI: $10.1016 /$ j.mechatronics.2018.03.002

[5] A. Schück, R. Labruyère, H. Vallery, R. Riener, and A. Duschau-Wicke, "Feasibility and effects of patient-cooperative robot-aided gait training applied in a 4-week pilot trial", Journal of NeuroEngineering and Rehabilitation, vol. 9, no. 31, 2012, ISSN: 1743-0003. DOI: 10.1186/1743-0003-9-31.

[6] B. M. Fleerkotte, B. Koopman, J. H. Buurke, E. H. F. van Asseldonk, H. van der Kooij, and J. S. Rietman, "The effect of impedance-controlled robotic gait training on walking ability and quality in individuals with chronic incomplete spinal cord injury: An explorative study", Journal of NeuroEngineering and Rehabilitation, vol. 11, no. 26, 2014, ISSN: 17430003. DOI: 10.1186/1743-0003-11-26.

[7] A. De Luca, H. Vernetti, C. Capra, I. Pisu, C. Cassiano, L. Barone, F. Gaito, F. Danese, G. Antonio Checchia, C. Lentino, P. Giannoni, and M. Casadio, "Recovery and compensation after robotic assisted gait training in chronic stroke survivors", Disability and Rehabilitation: Assistive Technology, pp. 1-13, 2018, ISSN: 17483115. DOI: $10.1080 / 17483107.2018$. 1466926 .

[8] W. H. Chang, M. S. Kim, J. P. Huh, P. K. Lee, and Y. H. Kim, "Effects of robot-assisted gait training on cardiopulmonary fitness in subacute stroke patients: A randomized controlled study", Neurorehabilitation and Neural Repair, vol. 26, no. 4, pp. 318-324, 2012, ISSN: 15459683. DOI: $10.1177 /$ 1545968311408916.

[9] E. Swinnen, N. Lefeber, W. Willaert, F. De Neef, L. Bruyndonckx, A. Spooren, M. Michielsen, T. Ramon, and E. Kerckhofs, "Motivation, expectations, and usability of a driven gait orthosis in stroke patients and their therapists", Topics in Stroke Rehabilitation, vol. 24, no. 4, pp. 299-308, 2017, ISSN: 19455119. DOI: 10.1080/10749357.2016.1266750.

[10] J. Meuleman, E. H. van Asseldonk, G. van Oort, H. Rietman, and H. van der Kooij, "LOPES II - Design and Evaluation of an Admittance Controlled Gait Training Robot with Shadow-Leg Approach", IEEE Transactions on Neural Systems and Rehabilitation Engineering, vol. 24, no. 3, pp. 352-363, 2016, ISSN: 15344320. DOI: 10.1109/TNSRE.2015.2511448.

[11] S. Srivastava, P. C. Kao, S. H. Kim, P. Stegall, D. Zanotto, J. S. Higginson, S. K. Agrawal, and J. P. Scholz, "Assist-as-Needed Robot-Aided Gait Training Improves Walking Function in Individuals Following Stroke", IEEE Transactions on Neural Systems and Rehabilitation Engineering, vol. 23, no. 6, pp. 956-963, 2015, ISSN: 15344320. DOI: 10.1109/TNSRE . 2014 . 2360822 . 
[12] A. Duschau-Wicke, A. Caprez, and R. Riener, "Patient-cooperative control increases active participation of individuals with SCI during robot-aided gait training", Journal of NeuroEngineering and Rehabilitation, vol. 7, no. 1 , pp. 1-13, 2010, ISSN: 17430003 . DOI: 10.1186/1743-0003-7-43.

[13] S. Maggioni, L. Lünenburger, R. Riener, and A. Melendez-Calderon, "Robot-Aided assessment of walking function based on an adaptive algorithm", IEEE International Conference on Rehabilitation Robotics, pp. 804-809, 2015, ISSN: 19457901. DOI: 10.1109/ICORR.2015.7281301.

[14] S. Maggioni, N. Reinert, L. Lünenburger, and A. Melendez-Calderon, "An Adaptive and Hybrid End-Point/Joint Impedance Controller for Lower Limb Exoskeletons", Frontiers in Robotics and AI, vol. 5, no. 104, 2018, ISSN: 1344-3542. DOI: 10.3389/frobt.2018.00104.

[15] C. Bayón, S. S. Fricke, E. Rocon, H. van der Kooij, and E. H. F. van Asseldonk, "Performance-Based Adaptive Assistance for Diverse Subtasks of Walking in a Robotic Gait Trainer: Description of a New Controller and Preliminary Results", Proceedings of the IEEE RAS and EMBS International Conference on Biomedical Robotics and Biomechatronics, pp. 414419, 2018, ISSN: 21551774. DOI: 10.1109/BIOROB.2018.8487189.

[16] J. L. Emken, S. J. Harkema, J. A. Beres-Jones, C. K. Ferreira, and D. J. Reinkensmeyer, "Feasibility of manual teach-and-replay and continuous impedance shaping for robotic locomotor training following spinal cord injury", IEEE Transactions on Biomedical Engineering, vol. 55, no. 1, pp. 322-334, 2008, ISSN: 00189294. DOI: 10.1109/TBME.2007.910683.

[17] S. Hussain, P. K. Jamwal, M. H. Ghayesh, S. Q. Xie, S. Member, and P. K. Jamwal, "Robust Nonlinear Control of an Intrinsically Compliant Robotic Gait Training Orthosis", IEEE Transactions on Industrial Electronics, vol. 64 , no. 2 , pp. 1675-1685, 2012, ISSN: 02780046. DOI: $10.1109 /$ TIE.2016.2580123.

[18] M. Zadravec, A. Olenšek, M. Rudolf, N. Bizovičar, N. Goljar, and Z. Matjačić, "A novel robot-assisted training approach for improving gait symmetry after stroke", IEEE International Conference on Rehabilitation Robotics, pp. 222-227, 2017, ISSN: 19457901. DOI: 10.1109/ICORR. 2017. 8009250 .

[19] D. Zanotto, P. Stegall, and S. K. Agrawal, "Adaptive assist-as-needed controller to improve gait symmetry in robot-assisted gait training", Proceedings - IEEE International Conference on Robotics and Automation, pp. 724-729, 2014, ISSN: 10504729. DOI: 10.1109/ICRA.2014.6906934.

[20] H. J. Asl and J. Yoon, "Stable assist-as-needed controller design for a planar cable-driven robotic system", International Journal of Control, Automation and Systems, vol. 15, no. 6, pp. 2871-2882, 2017, ISSN: 20054092. DOI: 10. 1007/s12555-016-0492-x. 
[21] B. Koopman, E. H. F. van Asseldonk, and H. van der Kooij, "Selective control of gait subtasks in robotic gait training: Foot clearance support in stroke survivors with a powered exoskeleton", Journal of NeuroEngineering and Rehabilitation, vol. 10, no. 1, 2013, ISSN: 17430003. DOI: 10.1186/17430003-10-3.

[22] J. M. Frullo, J. Elinger, A. U. Pehlivan, K. Fitle, K. Nedley, G. E. Francisco, F. Sergi, and M. K. O'Malley, "Effects of Assist-As-Needed Upper Extremity Robotic Therapy after Incomplete Spinal Cord Injury: A Parallel-Group Controlled Trial", Frontiers in Neurorobotics, vol. 11, no. 26, pp. 1-18, 2018. DOI: $10.3389 /$ fnbot .2017.00026.

[23] A. G. Grosmaire and C. Duret, "Does assist-as-needed upper limb robotic therapy promote participation in repetitive activity-based motor training in sub-acute stroke patients with severe paresis?", NeuroRehabilitation, vol. 41, no. 1, pp. 31-39, 2017, ISSN: 18786448. DOI: $10.3233 / \mathrm{NRE}-171454$.

[24] S. Y. A. Mounis, N. Z. Azlan, and S. Fatai, "Progress based assist-asneeded control strategy for upper-limb rehabilitation", Proceedings - 2017 IEEE Conference on Systems, Process and Control, ICSPC 2017, pp. 6570, 2017. DOI: 10.1109/SPC.2017.8313023.

[25] Y. Mao and S. K. Agrawal, "Design of a cable-driven arm exoskeleton (CAREX) for neural rehabilitation", IEEE Transactions on Robotics, vol. 28 , no. 4, pp. 922-931, 2012, ISSN: 15523098. DOI: 10.1109/TRO.2012. 2189496 .

[26] M. Mihelj, T. Nef, and R. Riener, "A novel paradigm for patientcooperative control of upper-limb rehabilitation robots", Advanced Robotics, vol. 21, no. 8, pp. 843-867, 2007, ISSN: 01691864. DOI: 10.1163/ 156855307780851975.

[27] L. H. Ting, H. J. Chiel, R. D. Trumbower, J. L. Allen, J. L. McKay, M. E. Hackney, and T. M. Kesar, "Neuromechanical principles underlying movement modularity and their implications for rehabilitation", Neuron, vol. 86, no. 1, pp. 38-54, 2015, ISSN: 10974199. DOI: 10.1016/j.neuron.2015.02. 042 .

[28] R. R. Neptune, D. J. Clark, and S. A. Kautz, "Modular control of human walking: A simulation study", Journal of Biomechanics, vol. 42, no. 9, pp. 1282-1287, 2009, ISSN: 00219290. DOI: $10.1016 /$ j . jbiomech . 2009 . 03.009 .

[29] J. Perry and J. M. Burnfield, Gait Analysis, Normal and Pathological Function. 1st ed. Thorofare, New Jersey: SLACK Incorporated, 1992, pp. 1-576, ISBN: 9781556421921. DOI: 10.2106/00004623-199303000-00027.

[30] M. A. Sharbafi, A. Seyfarth, and G. Zhao, "Locomotor Sub-functions for Control of Assistive Wearable Robots", Frontiers in Neurorobotics, vol. 11, no. 44, pp. 1-12, 2017, ISSN: 1662-5218. DOI: 10.3389/fnbot.2017.00044. 
[31] S. S. Fricke, C. Bayon, E. Rocon, H. van der Kooij, and E. H. F. van Asseldonk, "Pilot study of a performance-based adaptive assistance controller for stroke survivors", Biosystems and Biorobotics, vol. 21, pp. 302-306, 2018, ISSN: 21953570. DOI: 10.1007/978-3-030-01845-0_61.

[32] J. Meuleman, "Design of a Robot-Assisted Gait Trainer: LOPES II", PhD thesis, University of Twente, 2015, pp. 1-300, ISBN: 9789036539654. DOI: $10.3990 / 1.9789036539654$.

[33] C. Krishnan, D. Kotsapouikis, Y. Y. Dhaher, and W. Z. Rymer, "Reducing robotic guidance during robot-assisted gait training improves gait function: A case report on a stroke survivor", Archives of Physical Medicine and Rehabilitation, vol. 94, no. 6, pp. 1202-1206, 2013, ISSN: 00039993. DOI: 10.1016/j.apmr.2012.11.016.

[34] B. Koopman, E. H. F. van Asseldonk, and H. van der Kooij, "Speeddependent reference joint trajectory generation for robotic gait support", Journal of Biomechanics, vol. 47, no. 6, pp. 1447-1458, 2014, ISSN: 18732380. DOI: $10.1016 / \mathrm{j} \cdot \mathrm{jbiomech.2014.01.037.}$

[35] H.-S. Kim, S.-C. Chung, and M.-H. Choi, "Primary and secondary gait deviations of stroke survivors and their association with gait performance", The Journal of Physical Therapy Science Original, vol. 28, no. 9, pp. 26342640, 2016, ISSN: 0915-5287. DOI: 10.1589/jpts.28.2634.

[36] B. Balaban and F. Tok, "Gait Disturbances in Patients With Stroke", PM $R$, vol. 6 , no. 7 , pp. $635-642,2014$, ISSN: 19341482 . DOI: $10.1016 / \mathrm{j} \cdot \mathrm{pmrj}$. 2013.12.017.

[37] S. J. Olney and C. Richards, "Hemiparetic gait following stroke. Part I: Characteristics", Gait \& Posture, vol. 4, no. 2, pp. 136-148, Apr. 1996, ISSN: 09666362. DOI: 10.1016/0966-6362(96)01063-6.

[38] H. Barbeau, M. Ladouceq, K. E. Norman, A. Pépin, and A. Leroux, "Walking After Spinal Cord Injury: Evaluation, Treatment and Functional Recovery", Archiv, vol. 80, pp. 225-235, 1999. DoI: https://doi .org/10. 1016/S0003-9993(99)90126-0.

[39] H. Barbeau, S. Nadeau, and C. Garneau, "Physical Determinants, Emerging Concepts, and Training Approaches in Gait of Individuals with Spinal Cord Injury", Journal of Neurotrauma, vol. 23, no. 3, pp. 571-585, 2006. DOI: $10.1089 / \mathrm{neu} .2006 .23 .571$

[40] S. Armand, G. Decoulon, and A. Bonnefoy-Mazure, "Gait analysis in children with cerebral palsy", EFORT Open Reviews, vol. 1, no. 12, pp. 448 460, 2016, ISSN: 2396-7544. DOI: 10.1302/2058-5241.1.000052

[41] L. L. Cai, A. J. Fong, C. K. Otoshi, Y. Liang, J. W. Burdick, R. R. Roy, and V. R. Edgerton, "Implications of Assist-As-Needed Robotic Step Training after a Complete Spinal Cord Injury on Intrinsic Strategies of Motor Learning", Journal of Neuroscience, vol. 26, no. 41, pp. 10 564-10 568, 2006, ISSN: 0270-6474. DOI: 10.1523/JNEUROSCI . 2266-06.2006. 
[42] L. Marchal-Crespo and D. J. Reinkensmeyer, "Review of control strategies for robotic movement training after neurologic injury", Journal of NeuroEngineering and Rehabilitation, vol. 6, no. 1, p. 20, 2009, ISSN: 1743-0003. DOI: $10.1186 / 1743-0003-6-20$.

[43] R. Gassert and V. Dietz, "Rehabilitation robots for the treatment of sensorimotor deficits: A neurophysiological perspective", Journal of NeuroEngineering and Rehabilitation, vol. 15, no. 1, pp. 1-15, 2018, ISSN: 17430003. DOI: $10.1186 / \mathrm{s} 12984-018-0383-\mathrm{x}$.

[44] I. J. Park, J.-H. Park, H. Y. Seong, J. S. H. You, S. J. Kim, J. H. Min, H. Y. Ko, and Y.-I. Shin, "Comparative Effects of Different Assistance Force During Robot-Assisted Gait Training on Locomotor Functions in Patients with Subacute Stroke", American Journal of Physical Medicine $\&$ Rehabilitation, vol. 98, no. 1, pp. 58-64, 2018, ISSN: 0894-9115. DOI: 10.1097/phm.0000000000001027.

[45] J. A. Haarman, E. Maartens, H. Van Der Kooij, J. H. Buurke, J. Reenalda, and J. S. Rietman, "Manual physical balance assistance of therapists during gait training of stroke survivors: Characteristics and predicting the timing", Journal of NeuroEngineering and Rehabilitation, vol. 14, no. 1, pp. 1-11, 2017, ISSN: 17430003. DOI: 10.1186/s12984-017-0337-8.

[46] J. A. Galvez, A. Budovitch, S. J. Harkema, and D. J. Reinkensmeyer, "Quantification of therapists' manual assistance on the leg during treadmill gait training with partial body-weight support after spinal cord injury", Annual International Conference of the IEEE Engineering in Medicine and Biology - Proceedings, pp. 4028-4032, 2007, ISSN: 05891019. DOI: 10.1109/ IEMBS. 2007.4353217. 


\title{
Chapter 5
}

\section{Effects of selectively assisting impaired subtasks of walking in chronic stroke survivors}

S.S. Fricke, H.J.G. Smits, C. Bayón, J.H. Buurke, H. van der Kooij, E.H.F. van Asseldonk

This chapter has been submitted in February 2020 (under review).

\begin{abstract}
Recently developed controllers for robot-assisted gait training allow for the adjustment of assistance for specific subtasks (i.e. specific joints and intervals of the gait cycle that are related to common impairments after stroke). However, not much is known about possible interactions between subtasks and a better understanding of this can help to optimize (manual or automatic) assistance tuning in the future. In this study, we assessed the effect of separately assisting three commonly impaired subtasks after stroke: foot clearance (FC, knee flexion/extension during swing), stability during stance (SS, knee flexion/extension during stance) and weight shift (WS, lateral pelvis movement). For each of the assisted subtasks, we determined the influence on the performance of the respective subtask, and possible effects on other subtasks of walking and spatiotemporal gait parameters.

The robotic assistance for the FC, SS and WS subtasks was assessed in nine mildly impaired chronic stroke survivors while walking in the LOPES II gait trainer. Seven trials were performed for each participant in a randomized order: six trials in which either $20 \%$ or $80 \%$ of assistance was provided for each of the selected subtasks, and one baseline trial where the participant did not receive subtask-specific assistance. The influence of the assistance on performances for the assisted subtasks and other subtasks of walking as well as spatiotemporal parameters was analyzed.

Performances for the impaired subtasks (FC, SS and WS) improved significantly when assistance was applied for the respective subtask. Although WS performance improved when assisting this subtask, participants were not shifting
\end{abstract}


their weight well towards the paretic leg. On a group level, not many effects on other subtasks and spatiotemporal parameters were found. Still, performance for the leading limb angle subtask improved significantly resulting in a larger step length when applying FC assistance.

FC and SS assistance leads to clear improvements in performance for the respective subtask, while our WS assistance needs further improvement. As effects of the assistance were mainly confined to the assisted subtasks, tuning of FC, SS and WS can be done simultaneously. There is no need for specific, time-intensive tuning protocols (e.g. tuning subtasks after each other) in mildly impaired stroke survivors.

\subsection{Introduction}

Robot-assisted gait training (RAGT) has been developed to improve therapy after neurological disorders (e.g. stroke) by providing intensive and task-specific training while decreasing physical load for therapists. The use of robotic devices can positively affect gait training after stroke, especially when combined with common physical therapy and in the most impaired patients in the (sub-)acute phase after stroke 1]-3.

Previous studies suggest that RAGT can be further improved by personalizing training and promoting active participation since active participation is an essential factor in gait recovery and motor learning after stroke [4] -7 . To improve active participation, various controllers, based on the assist-as-needed principle (AAN, i.e. only assisting the patient when needed), have been developed [8]- 11 . Some of these current AAN controllers either set a specific assistance level for the whole gait cycle, or they adjust the assistance for each instance of the gait cycle (e.g. each percentage) [7], [9], [12]. Others focus on assisting specific joints and intervals of the gait cycle that are related to impairments after stroke (also called subtasks) [10, [11], 13], 14]. The assistance is changed for these subtasks based on deviations from reference trajectories [11], 13]. Assistance is applied for the (most) impaired subtasks, while subtask-based assistance allows the user to move freely during other, non-assisted, portions of the gait cycle.

A better understanding about the exact effect of subtask-based assistance on gait is needed to help with manual assistance tuning (i.e. tuning done by therapists), and optimize controllers that automatically tune assistance during RAGT. Recently, we developed an automatically-tuned subtask-based controller and tested it in people with stroke and spinal cord injury [13. This controller simultaneously adjusted the assistance for various subtasks of gait. However, whether interactions between subtasks affect this assistance tuning process is not known. For example, if the performance on one subtasks is limiting the overall gait performance, assistance on this subtask could lead to a widespread improvement on various other subtasks. In this case, only assistance on this 'bottleneck' subtask would be needed and not on each of the separate subtasks. We do not yet know whether these interactions occur and how they should be incorporated in the control.

In this study, we assessed the effect of assistance during walking for three of the most common impairments after stroke: 1. insufficient knee flexion during swing 
phase (foot clearance (FC) subtask), 2. increased knee flexion or hyperextension during stance phase (stability during stance (SS) subtask) and 3. problems with shifting the weight towards the paretic leg (weight shift (WS) subtask) [15], 16]. For each of these subtasks, only little is known about the effect on other intervals of the gait cycle and spatiotemporal parameters in stroke survivors:

Foot clearance $(F C)$ : Previous experiments in stroke survivors receiving foot clearance assistance in robotic gait trainers or using powered orthoses have also shown some effects, although minor, on other parts of the gait cycle. For example, foot clearance assistance in the LOPES I and LOPES II gait trainer resulted in an increase in knee and hip angles during the swing phase and a larger step height 12], [17. No significant effects on other spatiotemporal gait parameters were found in these studies. In addition, Sulzer et al. [18 found a small increase in peak hip abduction (2 deg.) when assisting knee flexion during the pre-swing phase with a powered knee orthosis (Series Elastic Remote Knee Actuator, SERKA).

Stability during stance (SS): To the best of our knowledge, so far no study investigated the effect of robotic assistance during the stance phase in stroke survivors [19]. In children with cerebral palsy, knee extension assistance did not lead to significant changes in step length and step width, however, an increase in peak stance knee and hip extension was found 20]. This shows the potential of SS assistance to also improve other aspects of walking.

Weight shift (WS): Only little is known about the effect of weight shift assistance. Next to improving lateral pelvis movement, weight shift assistance improved step length symmetry in a stroke survivor in LOPES II [10]. After spinal cord injury, weight shift assistance with another robotic device also led to an increased step length in the more impaired leg [21].

To sum up, sometimes small effects of assisting FC, SS and WS were found for specific subtasks in small groups of people with neurological disorders (1-9 participants per study). However, in most previous studies only a limited number of outcome measures were analyzed. The goal of the current study was to determine how robotic assistance for FC, SS and WS subtasks influences the gait pattern within one session. We analyzed the performance for various other subtasks and spatiotemporal gait parameters (e.g. step length, stance time). We expected that assistance for a specific subtask clearly improves performance for the assisted subtask, but could also influence other portions of the gait pattern. Findings from this study can lead to improvements in robotic gait training by optimizing assistance tuning and targeting assistance better towards the specific needs of the patient.

\subsection{Methods}

\subsubsection{Participants}

Nine chronic stroke survivors ( $>6$ months after stroke) participated in this study (7 male, $56 \pm 12$ years, height $1.78 \pm 0.06 \mathrm{~m}$, weight $83.4 \pm 8.9 \mathrm{~kg}$ ). Information about the participants, their clinical scores and settings while walking in LOPES II can be found in Table 5.1 .

The inclusion criteria were 1. diagnosis of a hemiparesis as a result of a stroke that occurred more than six months prior to the study, 2. a stable medical and 
Table 5.1: Overview of participants' characteristics, clinical scores and settings for LOPES II. ID: identification code used for each specific participant, 10MWT: 10 meter walking test, FAC: functional ambulation category, FMA: Fugl-Meyer assessment (lower extremity), MI: Motricity index (lower extremity), AFO: ankle foot orthosis, SCO: stance control orthosis.

\begin{tabular}{|c|c|c|c|c|c|c|c|c|c|c|c|c|}
\hline ID & Gender & Age & $\begin{array}{l}\text { Time post } \\
\text { stroke } \\
\text { (months) } \\
57\end{array}$ & $\begin{array}{l}\text { Paretic } \\
\text { leg } \\
\mathrm{r}\end{array}$ & $\begin{array}{l}\text { 10MWT } \\
(\mathrm{km} / \mathrm{h})\end{array}$ & $\begin{array}{lr}\text { Assistive } & \text { de- } \\
\text { vice(s) } & \text { used } \\
\text { during } & 10 \mathrm{MWT} \\
\end{array}$ & FAC & \multicolumn{2}{|c|}{ FMA MI } & $\begin{array}{l}\text { Walking } \\
\text { speed } \\
\text { LOPES } \\
(\mathrm{km} / \mathrm{h})\end{array}$ & $\begin{array}{l}\text { in } \\
\text { II }\end{array}$ & $\begin{array}{l}\text { Toe-lifter in } \\
\text { LOPES II }\end{array}$ \\
\hline $\mathrm{P} 2$ & $\mathrm{~m}$ & 61 & 28 & 1 & 2.4 & cane, AFO & 5 & 20 & 53 & 1.5 & & yes \\
\hline P3 & $\mathrm{m}$ & 74 & 55 & $\mathrm{r}$ & 4.0 & - & 5 & 28 & 64 & 1.5 & & no \\
\hline $\mathrm{P} 4$ & $\mathrm{~m}$ & 54 & 25 & $\mathrm{r}$ & 1.9 & cane, AFO & 5 & 11 & 28 & 1.2 & & yes \\
\hline P7 & $\mathrm{m}$ & 55 & 36 & 1 & 4.2 & - & 5 & 31 & 83 & 1.6 & & no \\
\hline P8 & f & 33 & 133 & 1 & 3.6 & - & 5 & 21 & 61 & 2.0 & & no \\
\hline P9 & f & 56 & 47 & $\mathrm{r}$ & 3.5 & $\mathrm{SCO}$ & 5 & 27 & 83 & 1.6 & & yes \\
\hline
\end{tabular}


physical condition that allowed for three minutes of walking, 3. an age of at least 18 years, and 4 . sufficient cognitive abilities to follow instructions and report any discomfort. Exclusion criteria were severe spasms that can interfere with the functions of LOPES II.

The experimental protocol was approved by the local medical ethical committee (METC Twente, Enschede, The Netherlands) and participants gave written informed consent prior to their participation.

\subsubsection{Robotic gait trainer and assistance}

The LOPES II robotic gait trainer was used in this study to provide robotic assistance to the participants during walking. LOPES II is a device that is attached to the user with push-pull rods at the pelvis and lower legs 10 . While the user is walking on an instrumented treadmill, the device can assist movements in eight degrees of freedom (DOFs): pelvis anteroposterior, pelvis mediolateral, and hip abduction/adduction, hip flexion/extension and knee flexion/extension for both legs. LOPES II is admittance controlled and the robot can be tuned from transparent mode ( $0 \%$ assistance, minimizing interaction forces between the device and the user) to full assistance (100\% assistance, mimicking position control).

LOPES II can move the user along reference trajectories when applying assistance. For each DOF, a reference trajectory is defined based on a data set from healthy, elderly subjects 22] and previous experiences with these reference trajectories [11, [13]. The amount of assistance that is applied by LOPES II depends on deviations from these reference trajectories and virtual spring stiffnesses (with equilibrium positions on the reference trajectories). This virtual spring stiffness $K$ is calculated with the following equation for each DOF $(j)$ and each instant $(i$ in $\%$ ) of the gait cycle:

$$
K_{j, i}=K_{\max , j}\left(\frac{G_{j, i}}{100}\right)^{2}
$$

where $K_{\max , j}$ is a maximal stiffness that is defined for each DOF of LOPES II and $G_{j, i}$ is the desired assistance [10].

For each DOF, a subtask-based assistance can be applied with LOPES II, i.e. assisting only specific intervals of the gait cycle for the respective DOF (e.g. for foot clearance only swing phase for the knee). In this study, we assisted the FC, SS and WS subtasks, as these subtasks are often the most impaired subtasks after stroke. The assistance profiles for these subtasks are shown in Table 5.2 together with other non-assisted subtasks that were used to assess the effect of assistance for FC, SS and WS. Each subtask was assisted separately at a low (20\%) and a high level (80\%), depending on the trial (see 5.2.3).

In addition to the subtask-based assistance, a general assistance was applied, meaning that the whole gait pattern was assisted (i.e. all DOFs during the whole gait cycle with a constant assistance level). The minimal impedance mode of LOPES II is not completely transparent and especially for the pelvis the virtual inertia is rather high (40kg) [10]. We applied a general assistance of $3 \%$ to make walking in the device without any additional assistance easier, especially for stroke survivors. 
Table 5.2: Overview of assistance and evaluated variables for different subtasks. Reference (black dotted lines) and example measured data (orange lines) positions and joint angles $\left(P_{\text {ref }}, P_{r e f}, \vartheta_{\text {ref }}, \vartheta_{\text {ref }}\right)$, assistance profiles $(K)$ and evaluation points (e.g. $\left.P_{\text {ref,e1 }}\right)$ that were used to calculate the error are shown. Each figure shows one gait cycle starting with left heel strike at 0\%. Weight shift to the right side, abduction and flexion angles are defined positive. The subtasks that were assisted in this study are shown with a grey background.

\begin{tabular}{|c|c|c|c|}
\hline Subtask (affected DOF) & $\begin{array}{l}\text { Reference } \\
\text { trajectory and } \\
\text { assistance profile }\end{array}$ & $\begin{array}{l}\text { Reference and } \\
\text { measured } \\
\text { trajectories, } \\
\text { evaluation points }\end{array}$ & Calculation of error \\
\hline $\begin{array}{l}\text { Foot clearance (knee } \\
\text { flexion during swing) }\end{array}$ & & ${ }^{\circ}$ & $\theta_{e r r}=\theta_{\text {ref }, e 1}-\theta_{\text {meas }, e 1}$ \\
\hline $\begin{array}{l}\text { Weight shift (lateral } \\
\text { pelvis position) }\end{array}$ & & & $P_{e r r}=\max \left(\left|P_{r e f, e 1}-P_{\text {meas }, e 1}\right|,\left|P_{\text {ref }, e 2}-P_{\text {meas }, e 2}\right|\right)$ \\
\hline
\end{tabular}

\section{Circumduction}

(maximal hip abduction angle)

\section{Lateral foot placement}

(hip abduction angle at $100 \%$ of gait cycle) not assisted

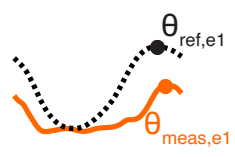

$$
\theta_{\text {err }}=\theta_{\text {meas }, e 1}-\theta_{\text {ref,e1 }}
$$

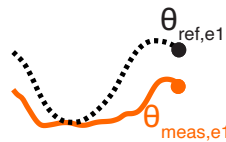

$$
\theta_{\text {err }}=\left|\theta_{\text {ref,e1 }}-\theta_{\text {meas }, e 1}\right|
$$


Trailing limb angle (minimal hip flexion angle)

Leading limb angle (maximal hip flexion angle)

Prepositioning (knee flexion at $100 \%$ of the gait cycle) not assisted

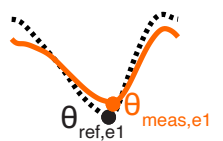

$$
\theta_{e r r}=\theta_{m e a s, e 1}-\theta_{r e f, e 1}
$$

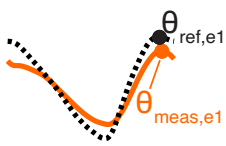

$$
\theta_{\text {err }}=\theta_{\text {ref }, e 1}-\theta_{\text {meas }, e 1}
$$

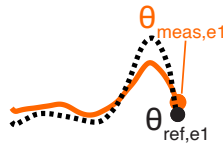

$\theta_{e r r}=\theta_{\text {meas }, e 1}-\theta_{\text {ref }, e 1}$ 


\subsubsection{Experimental procedures}

Each participant attended two sessions (familiarization and experimental session) on two different days. In the familiarization session, clinical assessment was performed by an experienced physical therapist. The following clinical scores were determined: Functional Ambulation Category (FAC) 23], comfortable walking speed in the 10 meter walking test (10MWT) [24, Fugl-Meyer Assessment (FMA) 25] for the lower extremity and Motricity Index (MI) 26 for the lower extremity (Table 5.1). After this, participants walked for three trials in LOPES II to get used to the device and to choose a comfortable walking speed. No body weight support was applied by the LOPES II system, however, participants used the hand rails of LOPES II during all trials in both sessions. In the first two trials of the familiarization session, participants received various amounts of general assistance (max. 30\%) to get used to LOPES II. In the last trial, $3 \%$ of general assistance was applied to make sure that they were able to walk in this condition. No individual assistance (FC, SS, WS) was applied in this familiarization session.

In the experimental session (Figure 5.1), participants started with a familiarization trial of 3 minutes to get used to walking in LOPES II again, receiving $3 \%$ general assistance. After this, seven trials (2 minutes each), which were used for data analysis in this study, were performed (Figure 5.1). The order of the trials was randomized between participants. In the baseline trial (BASE), no subtaskbased assistance was applied and participants received only $3 \%$ general assistance. In the other trials, $3 \%$ general assistance was applied for the whole gait cycle, and specific subtasks (FC, SS or WS) were assisted with either $20 \%$ or $80 \%$.

\begin{tabular}{|c|c|c|c|c|c|c|}
\hline BASE & FC20 & FC80 & SS20 & SS80 & WS20 & WS80 \\
\hline $\begin{array}{l}\text { No } \\
\text { assis- } \\
\text { tance }\end{array}$ & \multicolumn{2}{|c|}{$\begin{array}{c}\text { Foot } \\
\text { clearance } \\
\text { assistance }\end{array}$} & \multicolumn{2}{|c|}{$\begin{array}{c}\text { Stability } \\
\text { during stance } \\
\text { assistance }\end{array}$} & \multicolumn{2}{|c|}{$\begin{array}{l}\text { Weight shift } \\
\text { assistance }\end{array}$} \\
\hline
\end{tabular}

Figure 5.1: Overview of the seven trials of the experimental session. Each participant took part in all trials. The order of the seven trials was randomized and each trial took 2 minutes. BASE: baseline, WS: weight shift, FC: foot clearance, SS: stability during stance. No subtask-specific assistance was applied in BASE. 20 means 20\% of assistance and 80 means $80 \%$ of assistance for the respective subtask. For all other portions of the gait cycle $3 \%$ of general assistance was provided in all trials (including BASE).

\subsubsection{Outcome measures}

For each trial, the first five and last five steps were removed and not taken into account during further analyses. For each of the assisted subtasks, only participants who showed larger deviations than a specific threshold (FC: 7.54 deg., SS: 7.16 deg., WS: $2 \mathrm{~cm}$, Table 5.2 at the evaluation points in BASE were included in the analysis. These thresholds were based on average within trial standard deviations that were found in healthy participants while walking in minimal impedance mode 
in a previous study with LOPES II [1]. When participants showed smaller errors than these thresholds, they performed similar to healthy users and did not need the support. Including these well-performing participants would bias the outcome measures. Still, we performed all of the trials in those participants to use the same protocol in all participants.

Joint angles and joint positions were measured by LOPES II and sampled at $1000 \mathrm{~Hz}$ [10]. Based on these angles and joint positions, for each trial, we calculated the performance for various subtasks of gait and spatiotemporal parameters as described in the following paragraphs.

\section{Subtask performance}

To evaluate gait performance: FC, SS, WS, circumduction, lateral foot placement, trailing and leading limb angle, and prepositioning were used (Table 5.2). Subtaskbased performances were calculated based on deviations from reference trajectories for specific evaluations points (column 3 and 4 of Table 5.2).

For some subtasks, the absolute error was calculated as deviations in both directions can be found in stroke survivors. For example, for the SS subtask, some stroke survivors might have too much knee flexion during stance while others can get hyperextension of the knee. For other subtasks, we did not take the absolute error since we assumed that deviations in only one direction would be detrimental (Table 5.2): for example, a larger knee flexion during swing (foot clearance subtask) is acceptable. However, not enough knee flexion might lead to the toes being dragged along the ground which is detrimental for the walking pattern.

\section{Spatiotemporal parameters}

The following spatiotemporal parameters were assessed: step length (anteriorposterior distance between trailing and leading limb ankle at heel strike), step height (maximal ankle height during swing phase), step width (mediolateral distance between trailing and leading limb ankle at heel strike), and duration of the stance and swing phases.

\section{Statistics}

For each assisted subtask, Friedman tests were performed to assess differences between BASE and the two trials with assistance (20\% and $80 \%$ ) for subtask performances and spatiotemporal parameters. A p-value smaller than 0.05 was considered statistically significant. Post-hoc comparisons were performed by applying the Wilcoxon signed-rank test. A Bonferroni correction was used to account for multiple comparisons which means that p-values smaller than 0.017 (0.05 divided by 3 comparisons) were considered statistically significant for the post-hoc tests. 


\subsection{Results}

All participants performed all trials, however, one participant (P6) was not included for any of the results shown below as his performance was better than the threshold for each of the three assisted subtasks (FC, SS and WS) in BASE.

\subsubsection{Effect on subtask performance and spatiotemporal parameters Foot clearance (FC)}

For the FC subtask, seven of the nine participants showed an error larger than the threshold in BASE and were included in the analysis. Errors for the FC subtask significantly decreased when assistance was applied (Figure 5.3. $\left(\chi^{2}=14\right.$, $\mathrm{p}=0.0009))$. The average error decreased from $17.1 \mathrm{deg}$. in BASE to $7.9 \mathrm{deg}$. in FC20 and 1.7 deg. in FC80. Post hoc comparisons show significant differences for BASE-FC20, BASE-FC80 and FC20-FC80 ( $\mathrm{p}=0.0156$ in all three cases).

FC assistance also significantly affected the leading limb and trailing limb angle subtasks, showing consistent changes in various participants. The error for the leading limb angle subtask significantly decreased (i.e. hip flexion increased) when applying assistance $\left(\chi^{2}=11.14, \mathrm{p}=0.0038\right.$, posthoc: significant differences for BASE-FC80 and FC20-FC80 (both $\mathrm{p}=0.0156)$ ). However, it should be considered that in six of the seven participants the error was already close to 0 deg. or lower in BASE, and improvements for the leading limb angle subtask were not necessarily needed for these participants. For the trailing limb angle, significant differences were found $\left(\chi^{2}=6, p=0.0498\right)$. Although post hoc analyses showed a significant increase of the error between BASE and FC80 $(\mathrm{p}=0.0156)$, the effect was only small (about $1 \mathrm{deg}$.).

For the rest of the analysed subtasks, no significant effect on performances was found on a group level. Still, sometimes changes for other subtasks were found in specific participants (e.g. prepositioning for P4 and P9 in Figure 5.3.

Regarding the spatiotemporal parameters, significant effects on step length and step height of the paretic leg were found for FC assistance (Figure 5.2 step length: $\chi^{2}=12.29, \mathrm{p}=0.0021$, step height: $\left.\chi^{2}=7.14, \mathrm{p}=0.0281\right)$. Both, step length and step height were increased when more FC assistance was applied. For step length, a significantly increased paretic step length was found for FC assistance between BASE-FC20 and BASE-FC80 (in both cases p=0.0156). For step height, no significant differences were found for the posthoc tests.

In addition, a change in spatiotemporal parameters was observed for the (nonassisted) non-paretic leg: stance time was significantly affected $\left(\chi^{2}=6, p=0.0498\right)$. An increase in stance time from 1.60s in BASE to 1.65s in FC20 and FC80 was found. Posthoc tests did not show any significant differences for the pairwise comparisons.

\section{Stability during stance (SS)}

Seven of the nine participants needed assistance for the SS subtask (Figure 5.4). One of these participants (P8) had hyperextension in BASE while all other participants showed too much knee flexion during stance in BASE. 

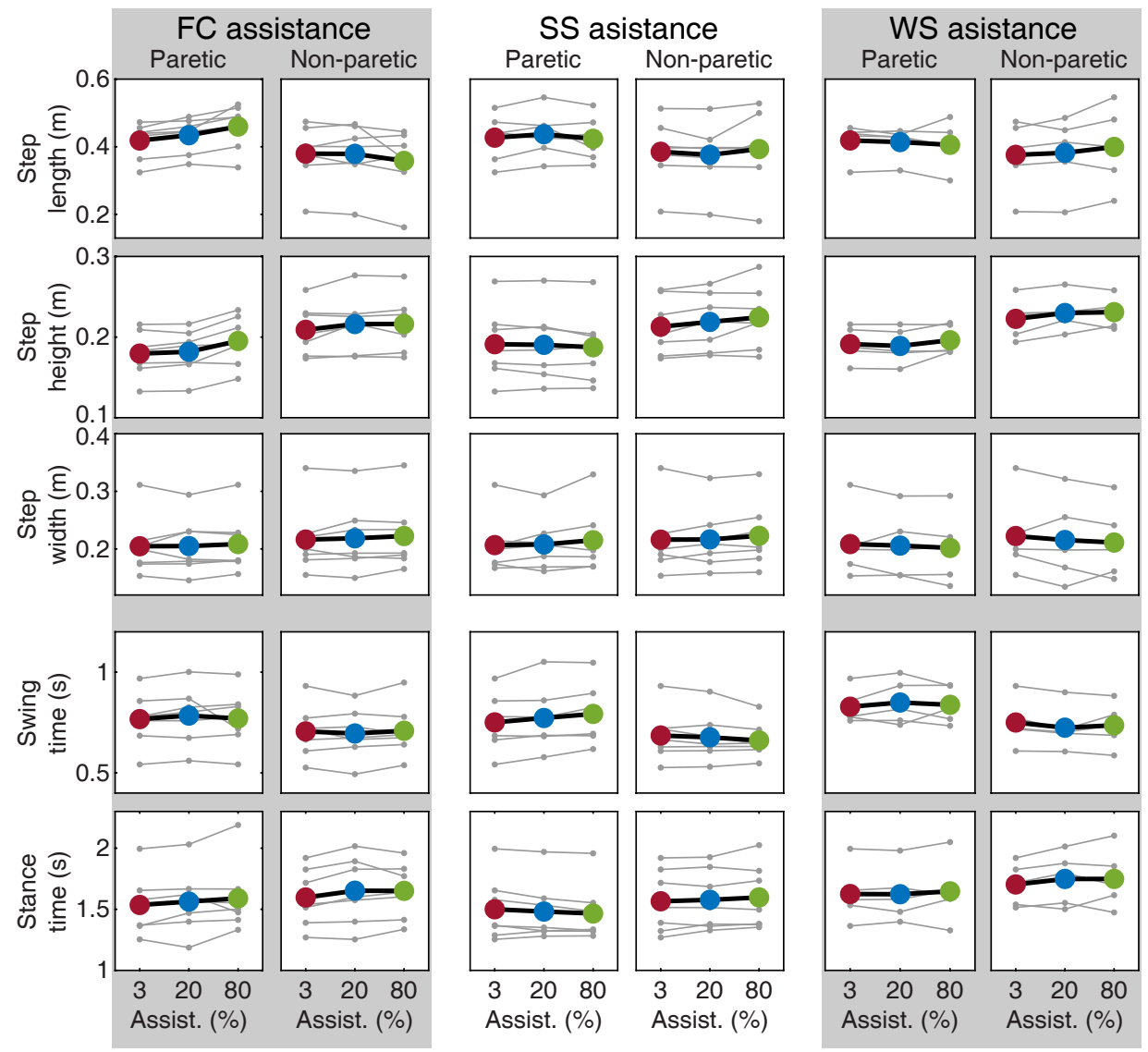

Figure 5.2: Effect of $F C, S S$ and WS assistance on spatial (top three rows) and temporal (bottom two rows) parameters. Only individuals that needed assistance for the respective subtask are shown in the columns for FC, SS and WS assistance. Each marker type and connecting grey line shows the results for one individual. The colored dots and connecting black lines show the average across participants for the respective parameter. Each black bar indicates significant differences between two conditions ( $a=0.05)$. 


\section{A (Trajectories)}
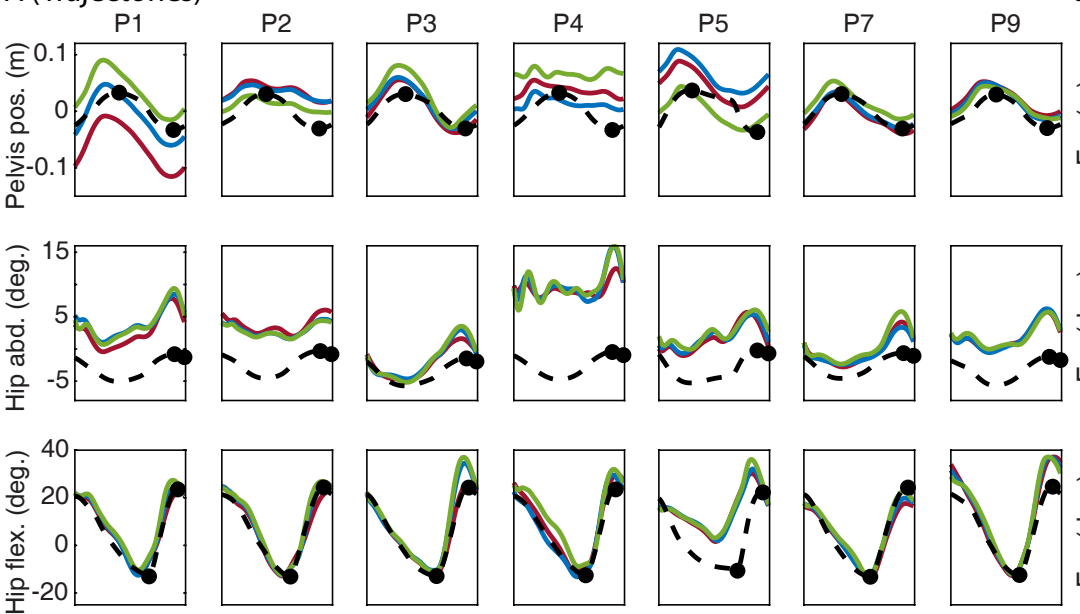

B (Errors)
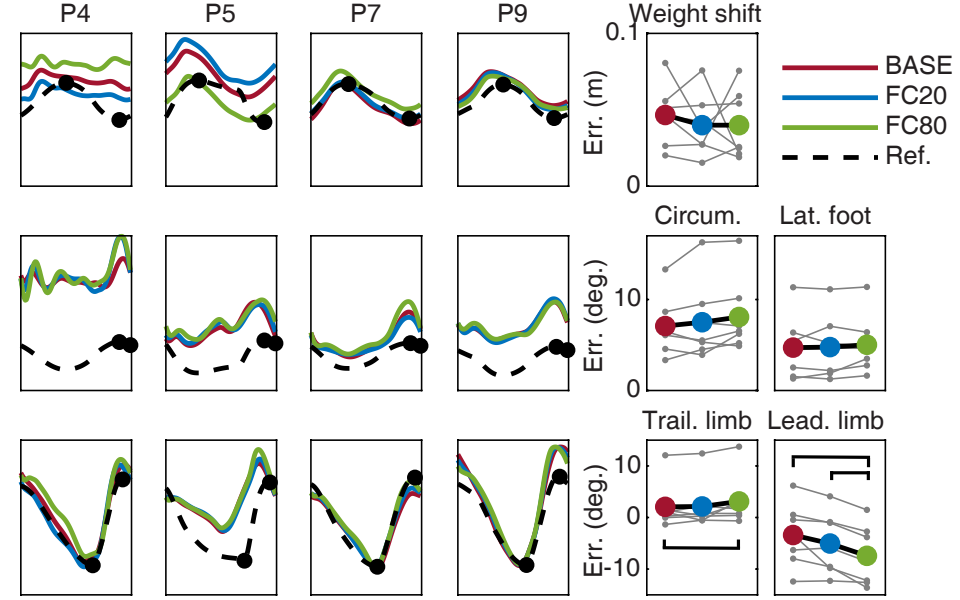

Lat. foot
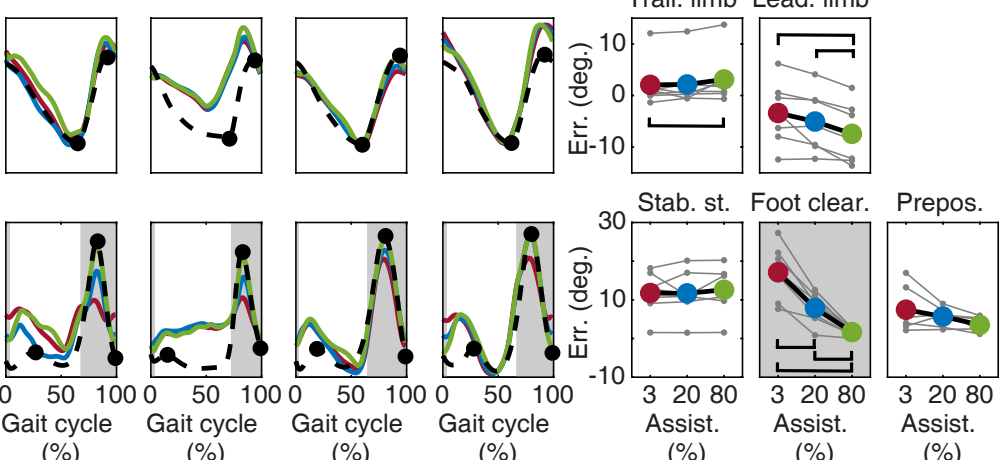

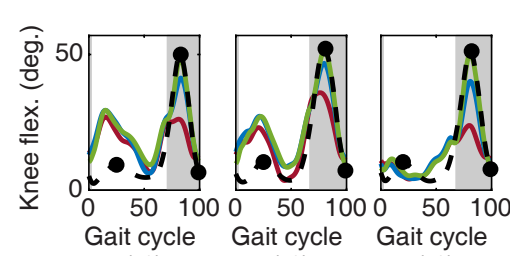

(\%)

(\%)

(\%)

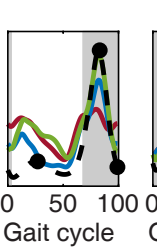

(\%)

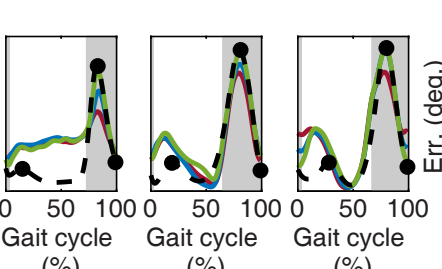

(\%)

(\%)

(\%)

(\%)

(\%)

(\%)

Figure 5.3: Joint trajectories (A) and errors (B) for each subtask for all individuals that needed FC assistance. Trajectories are shown for each individual separately. For the pelvis position, 0 is the center of the treadmill and a positive value means a deviation from the center towards the paretic side. Abduction and flexion angles are positive. The gait cycle starts with heel strike of the paretic leg. The grey area indicates the interval of the gait cycle within which the assistance was applied (A) and the assisted subtask (B). The black markers in $A$ indicate the evaluation points. For the weight shift, the largest error of the errors for both evaluation points is used to evaluate performance (see also Table 5.2. For the other DOFs the first marker corresponds to the first plot with errors, the second marker to the second plot with errors etc. Each grey marker and connecting grey line in B show the results for one individual. The colored dots and connecting black lines show the average errors across individuals. Each black bar indicates significant differences between two conditions (a=0.05). 


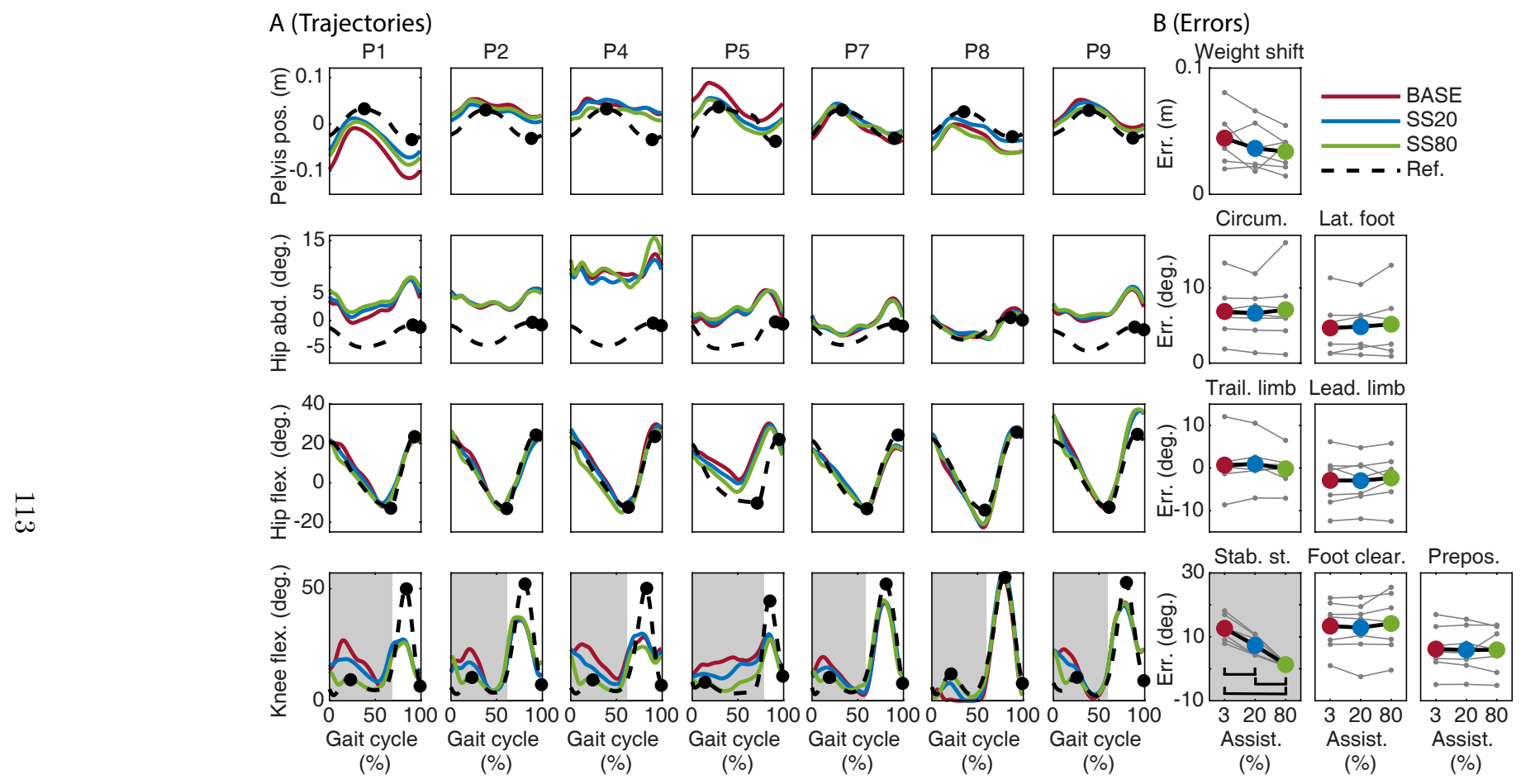

Figure 5.4: Joint trajectories (A) and errors (B) for each subtask for all individuals that needed SS assistance. Trajectories are shown for each individual separately. For the pelvis position, 0 is the center of the treadmill and a positive value means a deviation from the center towards the paretic side. Abduction and flexion angles are positive. The gait cycle starts with heel strike of the paretic leg. The grey area indicates the interval of the gait cycle within which the assistance was applied (A) and the assisted subtask (B). The black markers in A indicate the evaluation points. For the weight shift, the largest error of the errors for both evaluation points is used to evaluate performance (see also Table 5.2). For the other DOFs the first marker corresponds to the first plot with errors, the second marker to the second plot with errors etc. Each grey marker and connecting grey line in B show the results for one individual. The colored dots and connecting black lines show the average errors across individuals. Each black bar indicates significant differences between two conditions (a=0.05). 

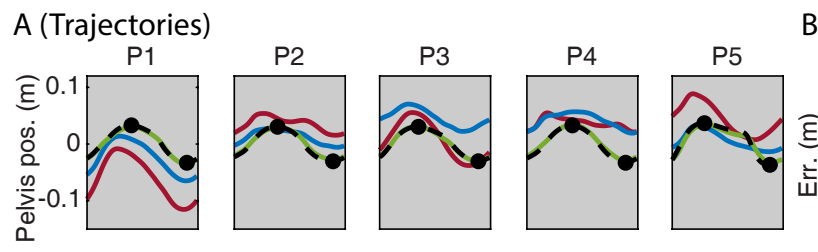

B (Errors)
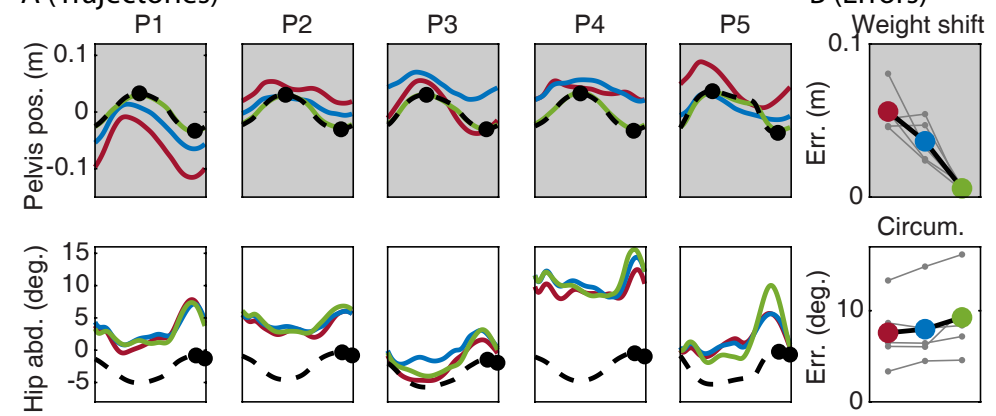

Lat. foot
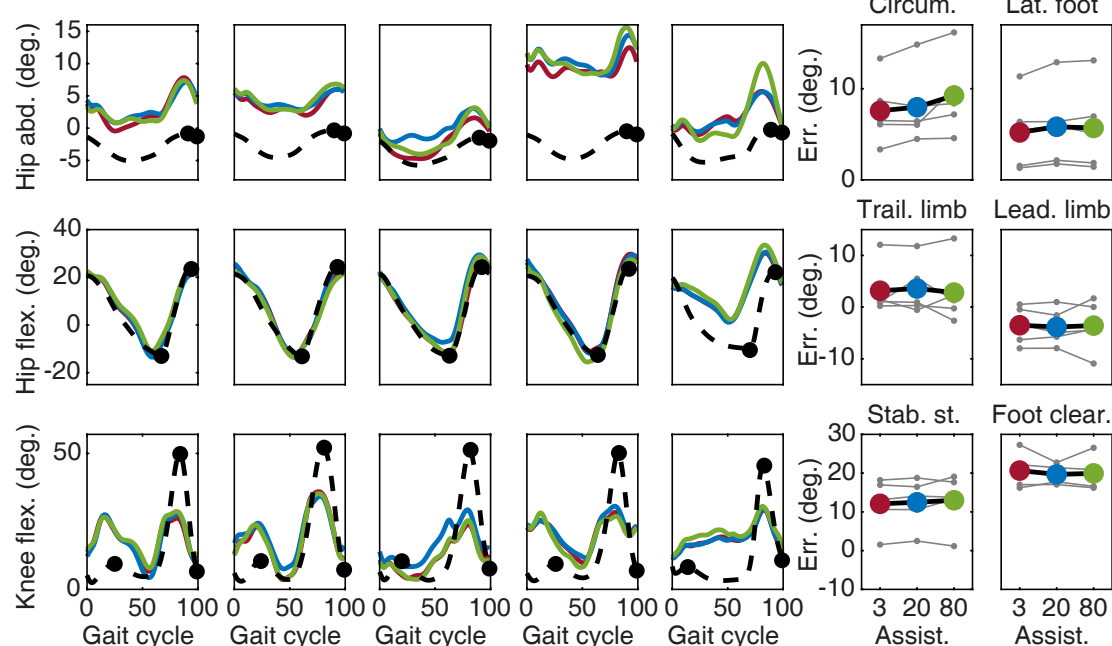

Gait cycle

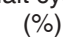

(\%)

(\%)

(\%)

(\%)

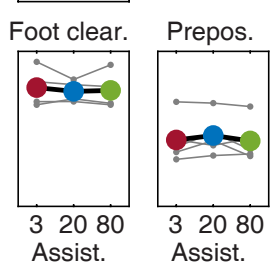

Figure 5.5: Joint trajectories (A) and errors (B) for each subtask for all individuals that needed WS assistance. Trajectories are shown for each individual separately. For the pelvis position, 0 is the center of the treadmill and a positive value means a deviation from the center towards the paretic side. Abduction and flexion angles are positive. The gait cycle starts with heel strike of the paretic leg. The grey area indicates the interval of the gait cycle within which the assistance was applied (A) and the assisted subtask (B). The black markers in A indicate the evaluation points. For the weight shift, the largest error of the errors for both evaluation points is used to evaluate performance (see also Table 5.2). For the other DOFs the first marker corresponds to the first plot with errors, the second marker to the second plot with errors etc. Each grey marker and connecting grey line in B show the results for one individual. The colored dots and connecting black lines show the average errors across individuals. Each black bar indicates significant differences between two conditions (a=0.05). 
The errors for the SS subtask significantly decreased by applying SS assistance $\left(\chi^{2}=14, \mathrm{p}=0.0009\right)$. Post hoc tests showed significant differences between BASESS20, BASE-SS80 and SS20-SS80 ( $p=0.0156$ in all cases). For none of the other subtasks a clear and significant effect of SS assistance was found on a group level.

No large changes for spatiotemporal parameters were found (Figure 5.2). Only swing time of the paretic leg was significantly influenced by SS assistance $\left(\chi^{2}=7.14\right.$, $\mathrm{p}=0.0281)$. Post hoc tests revealed a significant swing time increase of $0.04 \mathrm{~s}$ between BASE and FC80 ( $\mathrm{p}=0.0156)$.

\section{Weight shift (WS)}

Five participants were included for the assessment of WS assistance (Figure 5.5). WS assistance significantly affected performance for the WS subtask $\left(\chi^{2}=7.6\right.$, $\mathrm{p}=0.0224)$. Although participants were closer to reference trajectories in WS80 than in BASE or WS20 (Figure 5.5), post hoc comparisons did not show significant differences between any two of the three trials. In addition, no clear and consistent effect for all participants was found for the performance of any of the other subtasks.

The lateral pelvis movements were clearly affected by the WS assistance and all participants could closely follow the reference trajectory (Figure 5.5). However, this did not necessarily imply that participants were shifting their weight more towards the paretic leg. The mediolateral distance between the paretic ankle and the pelvis was much larger than the distance between the non-paretic ankle and the pelvis (Figure 5.6). Applying WS assistance moved the pelvis trajectory towards the reference trajectory, however, participants did not modify their foot placement relative to the pelvis. Consequently, for none of the participants, WS assistance resulted in changes in the distance between the ankle and pelvis for the paretic and non-paretic leg (Figure 5.7).

\subsection{Discussion}

The goal of this study was to determine the effect of FC, SS and WS assistance in LOPES II on the performance for the assisted subtasks, and to assess how this assistance affects the performance of other subtasks and spatiotemporal gait parameters. Performance for the assisted subtasks (FC, SS or WS) clearly improved when assistance was applied for the respective subtask. However, this selective assistance did hardly have beneficial effects on the performances of other non-assisted subtasks or spatiotemporal gait parameters. Only when applying FC assistance also the performance for the leading limb angle improved for the paretic leg, resulting in a larger step length. Still, the assistance also did not have significant detrimental effects on other intervals of the gait cycle, except for a small increase in the error for the trailing limb angle for $80 \% \mathrm{FC}$ assistance.

The lack of clear effects on other subtasks on a group level can have different causes. First, the patient population performed well for most unassisted subtasks, which means that no clear performance improvements were needed for these subtasks. However, as described below, participant-specific dependencies were found 


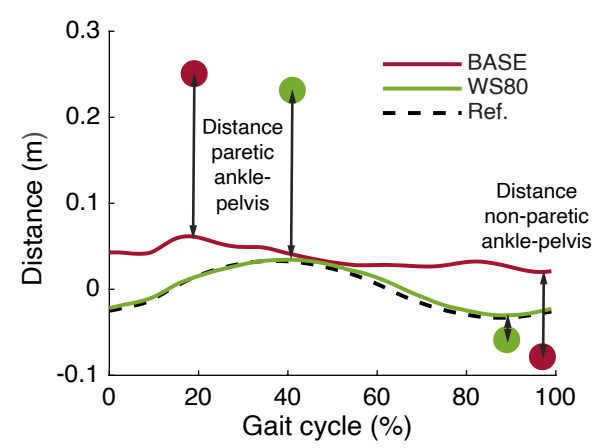

Figure 5.6: Example for the calculation of the distance between the ankles and pelvis. As an example, one step of BASE and one step of WS80 are shown. The line indicates the pelvis position and the markers indicate the position of the (paretic and nonparetic) ankle at that point of the gait cycle. $A$ distance of 0 means exactly in the center of the treadmill, the positive axis shows deviations from the center of the treadmill towards the side of the paretic leg. For each step, the distance between the pelvis and both ankles was calculated at the time instance of maximal and minimal lateral pelvis displacement as shown.

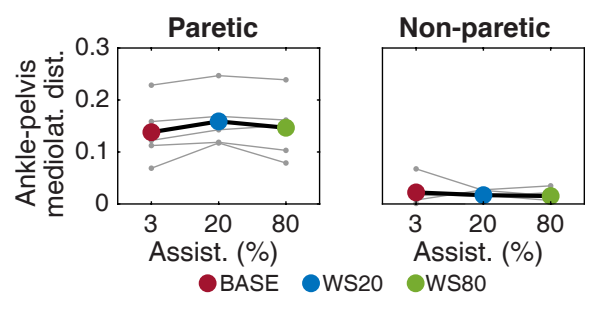

Figure 5.7: Distance between the pelvis and ankle (calculated as shown in Figure 5.6) for all individuals that needed WS assistance. A distance of 0 means exactly in the center of the treadmill, the positive axis shows deviations from the center of the treadmill towards the side of the respective leg. Each grey marker type shows the result for one individual. The coloured markers show the average for all included participants.

in some of the more impaired participants. Second, two of the most impaired subtasks, FC and SS, were assisted during different parts of the gait cycle (swing and stance, respectively), which makes interactions between these subtasks less likely.

\subsubsection{Comparison to literature}

Many of our results are in agreement with previous studies that evaluated the effect of FC, SS or WS assistance. However, the comparisons should be treated carefully due to the small sample sizes of the studies (including ours, max. 9 participants).

\section{Foot clearance (FC)}

For FC assistance, in line with our results, Koopman et al. [12 found an increased performance for the leading limb angle subtask in healthy participants. However, the results for the effect of FC assistance on hip abduction were less consistent: Koopman et al. 12 like us, did not find any significant differences in hip abduction when assisting FC during swing. In contrast to this, Sulzer et al. 18 found an increase in hip abduction when assisting knee flexion. One of the reasons for the differences could be that Sulzer et al. applied assistance during pre-swing phase 
which might have influenced the gait pattern in a different way than the assistance applied later during the swing phase in our study. Besides, it should be considered that we did not find a significant change in hip abduction on a group level. However, for 5 of the 7 participants we noticed more circumduction (i.e. more hip abduction during swing) for FC80 compared to BASE (Figure 5.3). Similar to results from Sulzer et al. [18, hip circumduction, which is sometimes seen as a compensatory mechanism in stiff-knee gait [15], did not decrease when applying knee flexion assistance during swing phase in our study. This also supports the results from a study by Akbas et al. [27] who claims that hip circumduction is not a compensatory mechanism for deficits in knee flexion during swing phase.

\section{Stability during stance (SS)}

For SS assistance, similar to a study by Lerner et al. [20 who applied assistance for the knee joint during the stance phase of walking in children with cerebral palsy, we did not find any significant effects on step length and step width. While Lerner et al. found an increase in peak hip extension during stance, in our study no clear changes in peak hip extension (trailing limb angle subtask) were found on a group level. A possible reason for this is that most of the stroke survivors already had sufficient hip extension in BASE (i.e. close to the reference trajectory) while children with crouch gait due to cerebral palsy do not have sufficient hip extension 28]. The one subject with limited hip extension in BASE in our study, did show an increase in peak hip extension resulting from SS assistance (P5 in Figure 5.4).

\section{Weight shift (WS)}

For WS assistance, our results deviate from previous pilot experiments with two stroke survivors walking in LOPES II [17], where WS assistance resulted in a decreased hip abduction. The reason for this is that the assistance is not directly comparable: Meuleman et al. 17] applied assistance for pelvis lateral movement as well as hip abduction during the whole gait cycle. In the current study, we wanted to focus on assistance of the lateral pelvis movement only, and did not assist hip abduction.

In our study, lateral pelvic movement assistance led to smaller deviations from the pelvis reference trajectory. However, users did not adjust their foot placement relative to the pelvis position, and therefore, did not shift their weight well towards the paretic leg. Assisting hip abduction, and ensuring proper lateral foot placement relative to the pelvis 17, can be a possible solution for this problem. Another approach is to adjust the reference trajectory for the pelvis based on foot placement. This means that the reference trajectory will be defined with respect to the leading stance foot to guarantee a certain weight shift, and not with respect to the global frame (center of treadmill) as currently done. 


\subsubsection{Study limitations}

\section{Patient population}

When assessing the effect of assistance for selective subtasks of gait, it is crucial that participants are able to walk without (or with low levels of) assistance, while still suffering from gait impairments that can effectively be supported. The reason for this is that a high general assistance would improve performance for all subtasks, making it impossible to clearly show effects of assisting one specific subtask. Due to these requirements, only mildly impaired stroke survivors could be included. Some of these participants already followed the reference trajectories well (low errors) for the FC, SS and/or WS subtask. Therefore, not all participants could be included for the analyses (maximum 7 out of 9 per subtask). Besides, the good performance for most of the unassisted subtasks left only little room for improvement on these subtasks which also makes it less likely to find interactions between subtasks. For more impaired participants in which several subtasks are affected, larger interactions could occur. This is illustrated by P5 in our study, who showed improved hip extension when applying SS assistance. Despite that it is difficult to find suitable participants that fulfill the above-mentioned requirements, we recommend to extend this research to more impaired participants.

\section{Handrail use}

Another limitation of this study is that participants were not able to walk without using the handrails as they did not feel secure enough. A previous study showed that handrail use while walking can influence step length and step width [29]. As participants used the handrails during all trails, we expected that handrail use did not have a large influence on the differences between trials. However, performance for weight shift is likely to be influence more by handrail use. Previous (unpublished) experiments with healthy participants walking in LOPES II in minimal impedance mode have shown limited lateral pelvic movements compared to the reference trajectory when using handrails.

\subsubsection{Future directions}

Our results can be used to optimize manual and automatic assistance tuning. In tuning support levels, we strive to quickly tune the assistance levels, and apply the least amount of assistance with the largest beneficial effects to enhance active participation of the user. Our ideal case would be to find the 'bottleneck' subtask of walking for each individual user. Only assisting this 'bottleneck' subtask would result in overall improvements of gait (positive effect in several other subtasks). We did not find many clear dependencies between subtasks in mildly impaired stroke survivors. Nonetheless, our results should be taken into account by therapists while manually tuning the assistance, and for the development of automaticallytuned controllers $[13$. FC, SS and WS can be tuned simultaneously without affecting each other and other non-assisted subtasks in mildly impaired stroke survivors. There is no need to use specific time-intensive 'tuning protocols' where, for example, subtasks are tuned after each other. 
In people with more impairments, and possibly more interactions between subtasks (e.g. similar to P5), advantages and disadvantages of various tuning protocols should be assessed. First, subtasks could still be tuned simultaneously to reduce the time needed for assistance tuning. Second, interactions between subtasks could be taken into account. For example, similar to how therapists tune the assistance [13], we would start tuning the most impaired subtask and apply low levels of assistance for other subtasks. If more improvements are needed, other subtasks should be tuned afterwards. Although it might be difficult to find suitable participants (see 5.4.2), future research is required to find an optimal tuning protocol for various subtask of gait in more impaired patients.

\subsection{Conclusion}

Separately assisting foot clearance and stability during stance enhances performance for the respective assisted subtask. Our weight shift assistance should be further improved in the future. Hardly any dependencies were found between the assisted subtasks and other subtasks as well as spatiotemporal parameters. Therefore, FC, SS and WS can be tuned simultaneously without affecting other subtasks in mildly impaired stroke survivors. No time-intensive tuning protocols (e.g. tuning subtasks after each other) are required in these patients.

\section{References}

[1] J. Mehrholz, S. Thomas, C. Werner, J. Kugler, M. Pohl, and B. Elsner, "Electromechanical-assisted training for walking after stroke (Review)", Cochrane Database of Systematic Reviews, no. 5, 2017. DoI: 10.1002/ 14651858. CD006185.pub4.

[2] M. F. Bruni, C. Melegari, M. C. De Cola, A. Bramanti, P. Bramanti, and R. S. Calabrò, "What does best evidence tell us about robotic gait rehabilitation in stroke patients: A systematic review and meta-analysis", Journal of Clinical Neuroscience, vol. 48, pp. 11-17, 2018, ISSN: 15322653. DOI: $10.1016 / \mathrm{j} \cdot$ jocn.2017.10.048.

[3] I. Schwartz and Z. Meiner, "Robotic-Assisted Gait Training in Neurological Patients: Who May Benefit?", Annals of Biomedical Engineering, vol. 43, no. 5, pp. 1260-1269, 2015, ISSN: 0090-6964. DOI: 10.1007/s10439-015$1283-\mathrm{x}$.

[4] S. F. Atashzar, M. Shahbazi, and R. V. Patel, "Haptics-enabled Interactive NeuroRehabilitation Mechatronics: Classification, Functionality, Challenges and Ongoing Research", Mechatronics, vol. 57, pp. 1-19, 2019, ISSN: 09574158. DOI: $10.1016 /$ j.mechatronics.2018.03.002.

[5] L. Marchal-Crespo and D. J. Reinkensmeyer, "Review of control strategies for robotic movement training after neurologic injury", Journal of NeuroEngineering and Rehabilitation, vol. 6, no. 1, p. 20, 2009, ISSN: 1743-0003. DOI: $10.1186 / 1743-0003-6-20$. 
[6] R. Gassert and V. Dietz, "Rehabilitation robots for the treatment of sensorimotor deficits: A neurophysiological perspective", Journal of NeuroEngineering and Rehabilitation, vol. 15, no. 1, pp. 1-15, 2018, ISSN: 17430003. DOI: $10.1186 / \mathrm{s} 12984-018-0383-\mathrm{x}$.

[7] J. L. Emken, S. J. Harkema, J. A. Beres-Jones, C. K. Ferreira, and D. J. Reinkensmeyer, "Feasibility of manual teach-and-replay and continuous impedance shaping for robotic locomotor training following spinal cord injury", IEEE Transactions on Biomedical Engineering, vol. 55, no. 1, pp. 322-334, 2008, ISSN: 00189294. DOI: 10.1109/TBME.2007.910683.

[8] S. Maggioni, L. Lünenburger, R. Riener, and A. Melendez-Calderon, "Robot-Aided assessment of walking function based on an adaptive algorithm", IEEE International Conference on Rehabilitation Robotics, pp. 804-809, 2015, ISSN: 19457901. DOI: 10.1109/ICORR.2015.7281301.

[9] S. Maggioni, N. Reinert, L. Lünenburger, and A. Melendez-Calderon, "An Adaptive and Hybrid End-Point/Joint Impedance Controller for Lower Limb Exoskeletons", Frontiers in Robotics and AI, vol. 5, no. 104, 2018, ISSN: 1344-3542. DOI: 10.3389/frobt.2018.00104

[10] J. Meuleman, E. H. van Asseldonk, G. van Oort, H. Rietman, and H. van der Kooij, "LOPES II - Design and Evaluation of an Admittance Controlled Gait Training Robot with Shadow-Leg Approach", IEEE Transactions on Neural Systems and Rehabilitation Engineering, vol. 24, no. 3, pp. 352-363, 2016, ISSN: 15344320. DOI: 10.1109/TNSRE.2015.2511448.

[11] C. Bayón, S. S. Fricke, E. Rocon, H. van der Kooij, and E. H. F. van Asseldonk, "Performance-Based Adaptive Assistance for Diverse Subtasks of Walking in a Robotic Gait Trainer: Description of a New Controller and Preliminary Results", Proceedings of the IEEE RAS and EMBS International Conference on Biomedical Robotics and Biomechatronics, pp. 414419, 2018, ISSN: 21551774. DOI: 10.1109/BIOROB.2018.8487189.

[12] B. Koopman, E. H. F. van Asseldonk, and H. van der Kooij, "Selective control of gait subtasks in robotic gait training: Foot clearance support in stroke survivors with a powered exoskeleton", Journal of NeuroEngineering and Rehabilitation, vol. 10, no. 1, 2013, ISSN: 17430003. DOI: 10.1186/17430003-10-3.

[13] S. S. Fricke, C. Bayón, H. van der Kooij, and E. H. F. van Asseldonk, "Automatic versus Manual Tuning of Robot-Assisted Gait Training in People with Neurological Disorders", Journal of NeuroEngineering and Rehabilitation, vol. 17, no. 9, 2020.

[14] G. Chen, J. Ye, Q. Liu, L. Duan, W. Li, Z. Wu, and C. Wang, "Adaptive control strategy for gait rehabilitation robot to assist-when-needed", 2018 IEEE International Conference on Real-Time Computing and Robotics, RCAR 2018, pp. 538-543, 2018. DOI: 10.1109/RCAR.2018.8621706

[15] B. Balaban and F. Tok, "Gait Disturbances in Patients With Stroke", PM $R$, vol. 6, no. 7, pp. 635-642, 2014, ISSN: 19341482. DOI: $10.1016 / \mathrm{j} \cdot \mathrm{pmrj}$. 2013.12.017. 
[16] S. J. Olney and C. Richards, "Hemiparetic gait following stroke. Part I: Characteristics", Gait \&3 Posture, vol. 4, no. 2, pp. 136-148, Apr. 1996, ISSN: 09666362. DOI: 10.1016/0966-6362(96)01063-6.

[17] J. Meuleman, "Design of a Robot-Assisted Gait Trainer: LOPES II", PhD thesis, University of Twente, 2015, pp. 1-300, ISBN: 9789036539654. DOI: 10.3990/1.9789036539654

[18] J. S. Sulzer, K. E. Gordon, Y. Y. Dhaher, M. A. Peshkin, and J. L. Patton, "Preswing Knee Flexion Assistance Is Coupled With Hip Abduction in People With Stiff-Knee Gait After Stroke", Stroke, vol. 41, no. 8, pp. 17091714, 2010, ISSN: 0039-2499. DOI: 10.1161/STROKEAHA.110.586917.

[19] B. Chen, B. Zi, Z. Wang, L. Qin, and W. H. Liao, "Knee exoskeletons for gait rehabilitation and human performance augmentation: A state-of-theart", Mechanism and Machine Theory, vol. 134, pp. 499-511, 2019, ISSN: 0094114X. DOI: 10.1016/j.mechmachtheory.2019.01.016.

[20] Z. F. Lerner, D. L. Damiano, and T. C. Bulea, "The effects of exoskeleton assisted knee extension on lower-extremity gait kinematics, kinetics, and muscle activity in children with cerebral palsy", Scientific Reports, vol. 7, no. 1, pp. 1-12, 2017, ISSN: 20452322. DOI: 10.1038/s41598-017-13554-2

[21] J. Lin, C. Hsu, W. Dee, D. Chen, W. Z. Rymer, and M. Wu, "Motor adaptation to weight shifting assistance transfers to overground walking in people with spinal cord injury", PmERR, 2019, ISSN: 1934-1482. DOI: $10.1002 / \mathrm{pmrj} .12132$

[22] B. Koopman, E. H. F. van Asseldonk, and H. van der Kooij, "Speeddependent reference joint trajectory generation for robotic gait support", Journal of Biomechanics, vol. 47, no. 6, pp. 1447-1458, 2014, ISSN: 18732380. DOI: $10.1016 / \mathrm{j} \cdot \mathrm{jbiomech} .2014 .01 .037$.

[23] M. Holden, K. Gill, M. Magliozzi, J. Nathan, and L. Piehl-Baker, "Clinical gait assessment in the neurologically impaired. Reliability and meaningfulness", Physical Therapy, vol. 64, no. 1, pp. 35-40, 1984, ISSN: 00319023. DOI: $10.1093 / \mathrm{ptj} / 64.1 .35$

[24] F. M. Collen, D. T. Wade, and C. M. Bradshaw, "Mobility after stroke: Reliability of measures of impairment and disability", Disability and Rehabilitation, vol. 12 , no. 1 , pp. 6-9, 1990, ISSN: 09638288. DOI: $10.3109 /$ 03790799009166594

[25] A. R. Fugl-Meyer, L. Jääskö, I. Leyman, and S. Olsson, "The post-stroke hemiplegic patient. 1. a method for evaluation of physical performance", Scandinavian journal of rehabilitation medicine, vol. 7, no. 1, pp. 13-31, 1975, ISSN: 0036-5505.

[26] D. T. Wade, Measurement in neurological rehabilitation, D. T. Wade, Ed., 2. Oxford: Oxford University Press, 1992, vol. 43, ISBN: 9780192621801. DOI: 10.1016/0960-8966(92)90066-F 
[27] T. Akbas, S. Prajapati, D. Ziemnicki, P. Tamma, S. Gross, and J. Sulzer, "Hip circumduction is not a compensation for reduced knee flexion angle during gait", Journal of Biomechanics, vol. 87, pp. 150-156, 2019, ISSN: 18732380. DOI: $10.1016 / \mathrm{j} \cdot \mathrm{jbiomech.2019.02.026}$.

[28] S. Armand, G. Decoulon, and A. Bonnefoy-Mazure, "Gait analysis in children with cerebral palsy", EFORT Open Reviews, vol. 1, no. 12, pp. 448460, 2016, ISSN: 2396-7544. DOI: 10.1302/2058-5241.1.000052.

[29] T. Ijmker, C. J. Lamoth, H. Houdijk, M. Tolsma, L. H. Van Der Woude, A. Daffertshofer, and P. J. Beek, "Effects of handrail hold and light touch on energetics, step parameters, and neuromuscular activity during walking after stroke", Journal of NeuroEngineering and Rehabilitation, vol. 12, no. 1, pp. 1-12, 2015, ISSN: 17430003. DOI: 10.1186/s12984-015-0051-3. 


\section{Chapter 6}

\section{General discussion}

The goal of this thesis was to take a next step towards personalized robot-assisted gait training. To achieve this goal, we defined two sub-goals:

1. Develop assessment tools to quantify walking function and underlying impairments affecting walking function (Chapters 2 and 3 ).

2. Improve subtask-based assistance and optimize assistance tuning based on users' walking abilities (Chapters 4 and 5).

In Chapter 2, we described assessment methods for joint impedance using a lower limb perturbator. We achieved first estimates of apparent hip joint impedance at different time points during the swing phase of walking in healthy participants. In the future, estimates of joint impedance can help to better understand the effect of RAGT on the neuromechanical system. In Chapter 3 , we tested a subtask-based controller that automatically adjusts assistance based on users' performance. This controller could be used for the assessment of walking function in a robotic gait trainer. However, both PBWS and walking speed affected the applied assistance. Therefore, this controller can only be used to quantify walking function and track progress if PBWS and walking speed are held constant for assessments. In Chapter 4, we compared manually-tuned (MT) to automaticallytuned (AT) subtask-based assistance in people with stroke and SCI. We found that automatic assistance tuning has several advantages above manual assistance tuning such as quick assistance tuning resulting in good performance for all subtasks. In Chapter 5. we showed that in mildly impaired stroke survivors various subtasks can be tuned simultaneously without affecting other subtasks. This means that no specific, time-intensive tuning protocols are needed (e.g. tuning subtasks after each other).

In the following sections we discuss our findings and implications regarding personalized RAGT, as well as future steps and recommendations in more detail. 


\subsection{Major findings and implications}

\subsubsection{Assessment of gait and underlying impairments using robotic devices}

Most previous developments for robotic gait trainers were aimed at (improving) therapy, and not at assessments. The Lokomat can be used for assessments such as joint position sense, range of motion, muscle strength and passive joint stiffness [1], 2]. However, these assessments are all performed in static situations (e.g. in a standing position). In order to get a more complete picture of impairments after neurological disorders and their effect on walking ability, it is important to perform assessments during walking. However, this leads to some additional challenges. First, devices should have negligible effects on movements of the user. Users need to be able to just continue walking while these assessments are performed. Second, severely impaired patients might not be able to walk without assistance. So, there is also a need for assessments that can result in meaningful outcomes when patients are assisted during walking. In this thesis, we described two assessment methods that can be applied during walking and are further discussed in the following paragraphs.

Modulation of joint impedance is crucial to be able to walk. Assessments are needed to get a better understanding of the modulation of joint impedance during walking 3. In Chapter 2, we developed the LOwer limb PERturbator (LOPER) which can be used to determine hip joint impedance during walking. Currently, this device overcomes one of the above mentioned challenges as only negligible effects on the walking pattern were observed for unperturbed parts of the gait cycle. We do not know yet whether the second challenge (i.e. assessment of joint impedance while assistance is applied) can also be addressed with this method. It should be investigated whether our joint impedance estimation method can also be used on top of assistive forces/torques. In addition, our experiments should be extended to people with neurological impairments. This will lead to a better understanding of how hip joint impedance (modulation) is affected after stroke and SCI, and how spasticity and hypertonia influence this impedance.

We determined first estimates of the apparent joint stiffness during the swing phase of walking, however, these estimates can be further improved. We did not estimate 'pure' hip joint impedance. The joint impedance of one joint (e.g. hip) can be influenced by other adjacent joints (e.g. knee) because of bi-articular muscles and heteronymous reflexes [4], [5]. Multiple-input multiple-output (MIMO) identification methods are needed to be able to determine pure hip and knee joint impedance as well as possible interactions between these joints. These methods require more advanced devices that can apply perturbations to multiple joints (hip and knee) 4]. Instead of directly starting with these more advanced devices, Chapter 2 was aimed at developing and evaluating a more simple device and approach to identify the apparent hip joint impedance. We considered this as a crucial step before advancing to more complex methods involving multiple perturbations.

In addition, more advanced system identification approaches can lead to a more detailed understanding of joint impedance, and the effect of RAGT on joint impedance. In Chapter 2, we have used a simple second order model to get a 
first estimate of apparent hip joint impedance during walking. This model did not take into account the different physiological contributions of joint impedance which are 1. the intrinsic contribution (due to limb inertia, viscoelastic properties of the joint, passive tissue, and passive and active muscle fibers) and 2 . the reflex contribution (due to neural reflex activity) 6], 7. . More advanced physiological models that include these two components have been used in previous studies [5], 7]-11. However, previously used system identification approaches that decompose joint impedance in the intrinsic and reflexive components are not suited for joint impedance estimations during walking. The reason for this is that these algorithms either do not take into account time-variant changes [8, 9] or can only detect slow time-variant changes [7, [10]. More advanced system identification algorithms are needed to be able to determine the contributions of the intrinsic and reflexive components to the total joint impedance during walking. This research could help to understand physiological changes that could occur after RAGT even better.

Before focusing on specific physiological changes after stroke/SCI and RAGT, it can be useful to assess walking function in general. Assessment of walking function using an AT controller is especially suited to overcome the second above mentioned challenge (i.e. assessment while assistance is applied). The users' walking ability could be evaluated by determining the minimal amount of assistance provided by an AT controller [2]. A high amount of assistance indicates that the users' walking ability is clearly diminished while no/a low amount of assistance means that the user is able to walk well with no/minimal assistance. The outcome measure of previous AT controllers was the amount of assistance for various bins over the complete gait cycle [12]. This resulting assistance is difficult to interpret by therapists and patients as it is not directly related to specific functional aspects of gait.

To overcome this, in Chapter 3, we developed an AT subtask-based controller. This controller adjusts assistance for several subtasks of walking which are related to common impairments after stroke and SCI (Table 1.1). The outcome measure of this controller is the assistance, i.e. virtual stiffness, for each subtask and leg. This outcome measure is easier to interpret than an outcome measure that is only related to specific bins of the gait cycle. Still, this subtask-based approach leads to a rather large number of outcome values as in total up to 13 subtasks can be assisted (Table 1.1). For ease of interpretation, it could be chosen to sort these subtasks based on the amount of assistance that is applied. If needed, therapists and/or patients can then focus on the most impaired subtask(s) only.

The AT subtask-based assessment was developed for LOPES II. Yet, it is very well suited to be implemented in other devices. Direct comparison of the outcome measures derived from these different devices will be cumbersome. The reason for this is that many differences can be found between robotic gait trainers regarding aspects such as transparency or degrees of freedom [13]-17]. For example, limitations in the degrees of freedom can possibly result in other (compensatory) movements that might affect the outcome measures. It needs to be investigated what the exact influence of differences between devices on the gait pattern is and whether using the same assessment in different devices results in similar outcome 
measures. We expect that future hardware and software improvements for robotic gait trainers can further decrease the influence of the device properties on outcome measures.

AT assistance and thus the outcome measures of this assessment are critically dependent on the reference trajectories. The predefined reference trajectories might not be optimal in several ways. First, the reference trajectories are based on average trajectories 18. As shown in Chapter 3, even the gait pattern of some unimpaired individuals deviates from these reference trajectories. This means that in some individuals assistance might be applied, even though they are able to walk without assistance. Second, reference trajectories were recorded during treadmill walking without PBWS, and were scaled based on walking speed 18. In Chapter 3, we have shown that factors such as PBWS and walking speed still might influence measured deviations from reference trajectories. Also, transparency and degrees of freedom of robotic gait trainers can affect deviations from reference trajectories. Third, it is not clear yet which reference patterns are optimal for people after neurological disorders. Reference patterns that take into account possible limitations (e.g. range of motion) and compensation mechanisms might be beneficial for training in this population. All of these limitations make it difficult to use AT assistance as an assessment tool, and compare different patients and walking conditions with each other. For now, the AT algorithm could be used to track progress within specific patients if the same conditions, such as device, walking speed and PBWS, are used for all sessions.

It should be ensured that automatically-tuned controllers result in minimal assistance that is needed to walk. Otherwise, if slacking occurs, the applied assistance would not be a measure of the real capacities of the user. We have determined the thresholds for increasing/decreasing the assistance based on gait variability within healthy participants. However, more research is needed to determine whether our current AT controller really results in the minimal assistance that is needed to be able to walk.

To sum up, we developed assessment methods for joint impedance and walking function (based on required assistance). The assessment of walking function is especially suited for more severely affected patients who need assistance during walking. It could be easily integrated into a training session. A high amount of assistance could indicate that the user has problems such as muscle weakness, deficiencies regarding control of muscles, or an impaired joint impedance (i.e. increased stiffness). Assessments of joint impedance can then give a better understanding of possible underlying impairments affecting gait. However, further developments are needed to show that this joint impedance assessment method can also be used in patients who are not able to walk without assistance.

\subsubsection{Personalized (subtask-based) assistance}

\section{Applied assistance and active participation}

Active participation and gait variability are important factors for neurorehabilitation after stroke and SCI. Active participation can possibly be enhanced by personalizing robotic gait assistance $[19-21$. To achieve this, we have developed 
an automatically-tuned subtask-based controller that adjusts assistance for various subtasks of gait based on users' performance (Chapters 3 and 4 ). In the following paragraphs we discuss this controller in more detail, and give some considerations regarding previous and future studies about RAGT.

There are several indications that our AT subtask-based controller promotes active participation and gait variability. First, the final assistance levels reached with the AT approach were often lower compared to MT assistance. We expect that AT assistance leads to more active participation than MT assistance. Second, when participants were performing well, assistance was decreased by the AT controller. We expect that this prevents slacking. Third, the AT controller only changed assistance if deviations from the reference trajectory were larger than a specific threshold allowing for some gait variability. Even though there are some indications that our controller promotes active participation and gait variability, it should be considered that we did not specifically assess this. To be able to give more conclusive evidence, active participation and gait variability should be investigated in the future, e.g. by measuring muscle activity and/or energy consumption.

There are several possibilities to further improve our AT subtask-based assistance. Other variations from Figure 1.4 could also be included. A deadband [22]-24] could be added to our controller. Within this deadband no assistance is applied to increase spatial variability during walking. In addition, high levels of PBWS were used in the most impaired participants. In the future, PBWS should be decreased as this can lead to more active participation and increased afferent feedback from load receptors which is crucial for gait rehabilitation [25]. Automatically tuning PBWS, simultaneously with the assistance levels $[12$, might help to easier test different combinations of PBWS and assistance levels. However, this is not possible with the current version of LOPES II. Hardware adjustments would be needed to be able to automatically tune PBWS.

The findings of Chapter 4 might have some implications for previously performed clinical trials. To our knowledge, AT assistance was not clinically implemented yet. Taking into account that AT assistance was often lower than MT assistance, it might be that patients received more assistance than needed in previous (clinical) studies where assistance was tuned manually. In Chapters 3 and 4 assistance was applied for specific subtasks, while other subtasks received low/no assistance. Assistance in previous clinical trials was not adjusted per subtask, but the whole gait cycle was assisted for all actuated joints, i.e. in most cases hip and knee joint [26-34]. This resulted in assistance for more parts of the gait cycle than needed. Also, it is likely that the amount of assistance depended on the most impaired subtask of gait in previous clinical studies. This would imply that other, less impaired, subtasks received more assistance than needed.

To personalize RAGT, it is important to get more insight into the amount of assistance that was applied in previous studies and how this affected clinical outcomes. However, information regarding the exact amount of assistance is often insufficient. Most clinical studies only describe the general protocol that was used to adjust walking speed, PBWS and assistance levels [26- 32 . These parameters were tuned based on visual assessments of patients capabilities. No exact assistance 
levels for specific patients were given in most previous clinical studies about RAGT [26-32]. For future clinical studies, we recommend to provide more information about the way that assistance is applied for each specific patient (e.g. timing, amount of assistance). An example of this is already provided in [35], where training duration, walking speed, PBWS and assistance levels are given for each participant. As the training effect can largely depend on the exact assistance that is applied [36], more detailed information about the assistance can help to understand possible differences in outcomes between clinical studies, and personalize RAGT in the future.

\section{Automatic and manual assistance tuning}

Despite that we found various advantages of the AT approach compared to the MT approach, we would not recommend to only use automatic tuning of robotic gait assistance. In Chapter 4, we have shown that the AT approach leads to faster assistance tuning, adjusts assistance for each subtask separately and results in lower assistance levels and good performance for all subtasks. Still, other, unstudied, factors that are not incorporated in the AT algorithm should also be considered during assistance tuning. First, therapists can directly use feedback from patients to tune the controller. Problems such as discomfort that can especially occur during long training sessions can be directly tackled by therapists. Second, our current AT controller tries to achieve movements similar to the reference trajectories. In contrast to this, therapists could choose to allow compensatory strategies which are important for the recovery of gait [37]. To sum up, we recommend to combine automatic and manual assistance tuning in the future to 1 . take into account feedback from users, 2. include therapists' knowledge, and 3. quickly tune assistance and achieve good performance for all subtasks.

AT and MT assistance can be combined in different ways. First, an AT controller can be used to quickly and continuously (e.g. every three steps) tune the assistance. Therapists can manually override the automatically set assistance. Second, therapists could choose specific assistance ranges for each subtasks (e.g. no, low, medium, high). For example, a low assistance could be associated with assistance percentages from 10-30\%. An AT controller determines the exact amount of assistance within the chosen range. Third, the AT controller could recommend specific assistance levels. However, therapists still have to set assistance levels manually. They could either follow the recommendations of the AT controller, or choose assistance levels themselves. It needs to be investigated whether any of these possible combinations of automatic and manual tuning results in the most benefits for therapists as well as patients.

Irrespective of the specific combination of AT and MT assistance that might be chosen in the future, we should aim for quick assistance tuning. This ensures that patients can exercise as long as possible at their personalized assistance levels. In Chapter 5, we have shown that in mildly impaired stroke survivors various subtasks (foot clearance, stability during stance, weight shift) can be tuned simultaneously without affecting other subtasks. No specific, time-intensive tuning protocols, such as tuning subtasks after each other (starting with the most impaired subtask), are 
needed. This also means that our developed AT controller, which tunes assistance for all subtasks simultaneously, does not need to be adjusted for mildly impaired stroke survivors. However, we do not know yet whether specific subtasks influence other subtasks in more impaired individuals with stroke/SCI. If these individuals show clear interactions between subtasks, more research is needed on the optimal tuning protocol. In this case, 1. assistance can be tuned like a therapist would do, by first focusing on the most impaired subtasks, or 2. still all subtasks could be tuned simultaneously, possibly resulting in faster assistance tuning.

\section{Other applications for automatic assistance tuning}

AT controllers can also be a valuable tool in educating therapists. The number of rehabilitation robotics is increasing 21]. Therapists need to learn how to use several devices. For some of these devices, assistance tuning can be complex and difficult to learn. Even when subtask-based assistance is used for RAGT, still up to 13 assistance levels can be tuned (Table 1.1). Especially for novice users this assistance tuning can be difficult and time-intensive. An AT controller could be used to show these therapists how the assistance can be tuned. Based on our results from Chapter 4 , we expect that AT controllers can especially demonstrate therapists that low assistance levels can result in good performance and can be safely used.

\subsection{Future steps and recommendations}

Studies comparing various controllers for RAGT in a broad patient population, especially on the long-term, are scarce 38]. Most previous clinical studies compared (impedance-based) RAGT with either physical therapy alone, or a combination of RAGT and physical therapy [39- 44 . More advanced controllers that potentially increase gait variability and active participation were often only tested in healthy participants and/or a low number of people with neurological disorders [12]- 14], 22], 23], 35], 45]-50. These studies were aimed at first tests with a new controller in a small number of participants. Therefore, often no control group receiving assistance by a different controller was used [13, 22], [23], 35], 45]. Only some studies compare multiple controllers regarding short term effects. Most of these studies show promising results such as an increased active participation for more advanced controllers [14], [47]-[50]. More short-term, and especially long-term studies in broad patient populations are needed. Different control algorithms, such as personalized (AT and MT) subtask-based robotic gait assistance, should be compared. These studies could provide more evidence about the best way to tune assistance for RAGT in the future.

The effect of RAGT is not only influenced by the chosen control algorithm and amount of assistance. Other training parameters (PBWS, walking speed) and treatment protocols (number of training hours, frequency and duration of training sessions) can influence outcomes for RAGT [51], 52]. For conventional training (i.e. physical therapy), a longer training duration resulting in more repetitions can improve training outcomes after stroke and SCI [51], 53]-55]. However, especially 
for RAGT, different combinations of several training parameters/treatment protocols can influence training intensity and training outcomes. Despite that some studies investigated the effect of specific parameters [47], 52], [56], so far, the optimal combinations of training parameters and treatment protocols are unknown. Future research should not only focus on comparing various controllers, but also take into account differences in other training parameters and treatment protocols.

Assessments as described in this thesis and other studies 11, 2] could help to quantify gait and underlying impairments, monitor changes during therapy, and also improve understanding of the exact physiological effect of RAGT. So far, most clinical studies use rather unspecific outcome measures such as walking speed, 10-meter walk test or 6-minute walk test [39], [57. Improvements for these outcome measures might occur for various reasons. Assessments of sensorimotor impairments, or specific impaired subtasks, could help to better understand why these outcome measures change after training for each specific patient. In the future, more specific outcome measures should be used to better understand the effect of robotic gait assistance, and to be able to adjust and personalize training protocols based on these results.

Assessments of other impairments than joint impedance can be used to further personalize RAGT and achieve a better understanding of physiological changes after RAGT. First, measurements of active and passive range of motion (ROM) can contribute to prevent injuries by ensuring that robotic gait trainers do not try to move patients in extreme positions out of their individual ROM. This measurement can already be performed with the Lokomat. Second, measurements of muscle strength and proprioception can also already be performed with the Lokomat and help to understand why gait function might have changed after RAGT. Third, an abnormal joint torque coupling between hip extension and adduction was found for the hip joint in a static position in stroke survivors [58]. If this coupling also occurs during walking, it might lead to instability during the stance phase where hip extension and hip abduction are required. Future research is needed to determine whether joint torque couplings during walking exist, and how this could be incorporated in the assistance tuning for RAGT. Fourth, other factors, e.g. balance [59], should also be taken into account to get a more complete picture of physiological changes after RAGT.

The AT subtask-based assistance that was used in LOPES II when walking on a treadmill could also be extended to other robotic gait trainers and to other gait-related tasks. In its current form, the controller can be used in devices that can measure joint angles and have a tunable joint impedance. These devices could be treadmill-based robotic gait trainers, but also robotic gait trainers or powered exoskeletons for overground walking [60, 61]. Currently, the controller can only assist with straight walking. The reference trajectories are based on straight treadmill walking, and thresholds to increase/decrease assistance are based on walking in LOPES II. The AT subtask-based controller could be extended to other tasks (e.g. turning, walking stairs) during overground walking by adjusting the reference trajectories and thresholds for increasing/decreasing the assistance.

In an ideal case, the same device can be used for training as well as assessment. This results in a low setup time for both therapists and patients. If assessments 
can be directly included in the training process, it allows to spend more time on the actual gait training. Unfortunately, current robotic gait trainers are in several ways not sophisticated enough to perform assessments of various impairments, while minimally influencing outcome measures. First, transparency needs to be further improved. Although healthy users show close to normal (i.e. free walking) gait patterns in LOPES II 13], it is still difficult to walk without handrails in minimal impedance mode due to the rather large virtual mass $(30 \mathrm{~kg})$ at the pelvis. Second, bandwidth is not high enough to apply the perturbations that are needed to determine joint impedance. Third, currently, devices such as the Lokomat and LOPES II cannot be used to perform assessments at the ankle joint. For a complete assessment of the lower limbs, actuation of the ankle joint is needed. ALEX III is a device that can already control the pelvis, hip, knee and ankle joints 17, 62. However, it is unknown how this device affects the gait pattern compared to free treadmill walking, and if the bandwidth is high enough for joint impedance estimates. To sum up, robotic gait trainers as well as assessment methods should be further evaluated and improved to be able to use these devices in an optimal way, not only for training, but also for assessments.

The development of controllers that personalize robotic gait assistance could lead to more effective RAGT for a broader range of patients. According to previous studies, robot-assisted gait trainers are most suited for severely impaired patients 61]. However, it should also be considered that assistance in previous clinical studies often was not personalized. We expect that RAGT has the potential to improve walking function in mildly impaired patients. Active participation should be ensured by personalizing assistance and using low levels of assistance. Also, controllers applying resistance [17], 63] or enhancing errors [49] can increase active participation while walking in robotic gait trainers.

\subsection{Final remarks}

In this thesis, we developed assessment methods and an automatically-tuned subtask-based controller that can be used to personalize robotic gait assistance. Despite that we have taken a next step towards personalized robotic gait training, we have also shown that there are still some unanswered questions and challenges to overcome. An important following step is to determine the long-term effect of various controllers. In addition, assessment tools should be used to better understand the exact physiological effects of robot-assisted gait training after stroke and SCI. More knowledge about the exact physiological changes after RAGT can help to get the most out of personalized robot-assisted gait training.

\section{References}

[1] A. Domingo and T. Lam, "Reliability and validity of using the Lokomat to assess lower limb joint position sense in people with incomplete spinal cord injury", Journal of NeuroEngineering and Rehabilitation, vol. 11, no. 1, pp. 1-10, 2014, ISSN: 17430003. DOI: 10.1186/1743-0003-11-167. 
[2] S. Maggioni, A. Melendez-Calderon, E. H. F. van Asseldonk, V. KlamrothMarganska, L. Lünenburger, R. Riener, and H. van der Kooij, Robot-aided assessment of lower extremity functions: A review, 2016. DOI: 10.1186/ s12984-016-0180-3.

[3] H. Lee, E. J. Rouse, and H. I. Krebs, "Summary of Human Ankle Mechanical Impedance during Walking", IEEE Journal of Translational Engineering in Health and Medicine, vol. 4, no. September, 2016, ISSN: 21682372. DOI: $10.1109 /$ JTEHM. 2016.2601613.

[4] B. Koopman, E. H. F. van Asseldonk, and H. van der Kooij, "Estimation of Human Hip and Knee Multi-Joint Dynamics Using the LOPES Gait Trainer", IEEE Transactions on Robotics, vol. 32, no. 4, pp. 920-932, 2016, ISSN: 15523098. DOI: 10.1109/TRO.2016.2572695.

[5] C. G. M. Meskers, J. H. de Groot, E. de Vlugt, and A. C. Schouten, "NeuroControl of movement: system identification approach for clinical benefit", Frontiers in Integrative Neuroscience, vol. 9, no. September, pp. 1-11, 2015. DOI: $10.3389 /$ fnint .2015.00048.

[6] M. M. Mirbagheri, H. Barbeau, and R. E. Kearney, "Intrinsic and reflex contributions to human ankle stiffness: Variation with activation level and position", Experimental Brain Research, vol. 135, no. 4, pp. 423-436, 2000, ISSN: 00144819. DOI: 10.1007/s002210000534.

[7] D. Ludvig and R. E. Kearney, "Real-time estimation of intrinsic and reflex stiffness", IEEE Transactions on Biomedical Engineering, vol. 54, no. 10, pp. 1875-1884, 2007, ISSN: 00189294. DOI: 10.1109/TBME.2007.894737.

[8] M. M. Mirbagheri, H. Barbeau, M. Ladouceur, and R. E. Kearney, "Intrinsic and reflex stiffness in normal and spastic, spinal cord injured subjects", Experimental Brain Research, vol. 141, no. 4, pp. 446-459, 2001, ISSN: 00144819. DOI: 10.1007/s00221-001-0901-z.

[9] M. M. Mirbagheri, L. Alibiglou, M. Thajchayapong, and W. Z. Rymer, "Muscle and reflex changes with varying joint angle in hemiparetic stroke", Journal of NeuroEngineering and Rehabilitation, vol. 5, pp. 1-15, 2008, ISSN: 17430003. DOI: 10.1186/1743-0003-5-6.

[10] R. C. van 't Veld, A. C. Schouten, H. van der Kooij, and E. H. F. van Asseldonk, "Validation of Online Intrinsic and Reflexive Joint Impedance Estimates Using Correlation with EMG Measurements", Proceedings of the IEEE RAS and EMBS International Conference on Biomedical Robotics and Biomechatronics, vol. 2018-Augus, pp. 13-18, 2018, ISSN: 21551774. DOI: 10.1109/BIOROB. 2018.8488123.

[11] C. G. Meskers, A. C. Schouten, J. H. De Groot, E. De Vlugt, B. J. Van Hilten, F. C. Van Der Helm, and H. J. Arendzen, "Muscle weakness and lack of reflex gain adaptation predominate during post-stroke posture control of the wrist", Journal of NeuroEngineering and Rehabilitation, vol. 6, no. 1, 2009, ISSN: 17430003. DOI: 10.1186/1743-0003-6-29. 
[12] S. Maggioni, L. Lünenburger, R. Riener, and A. Melendez-Calderon, "Robot-Aided assessment of walking function based on an adaptive algorithm", IEEE International Conference on Rehabilitation Robotics, pp. 804-809, 2015, ISSN: 19457901. DOI: 10.1109/ICORR.2015.7281301.

[13] J. Meuleman, E. H. van Asseldonk, G. van Oort, H. Rietman, and H. van der Kooij, "LOPES II - Design and Evaluation of an Admittance Controlled Gait Training Robot with Shadow-Leg Approach", IEEE Transactions on Neural Systems and Rehabilitation Engineering, vol. 24, no. 3, pp. 352-363, 2016, ISSN: 15344320. DOI: 10.1109/TNSRE.2015.2511448.

[14] T. Aurich-Schuler, F. Grob, H. J. Van Hedel, and R. Labruyère, "Can Lokomat therapy with children and adolescents be improved? An adaptive clinical pilot trial comparing Guidance force, Path control, and FreeD", Journal of NeuroEngineering and Rehabilitation, vol. 14, no. 1, pp. 1-14, 2017, ISSN: 17430003. DOI: 10.1186/s12984-017-0287-1.

[15] R. Riener, L. Lünenburger, I. C. Maier, G. Colombo, and V. Dietz, "Locomotor training in subjects with sensori-motor deficits: An overview of the robotic gait orthosis Lokomat", Journal of Healthcare Engineering, vol. 1, no. 2, pp. 197-216, 2010, ISSN: 20402309. DOI: 10.1260/2040-2295.1.2. 197.

[16] J. F. Veneman, R. Kruidhof, E. E. Hekman, R. Ekkelenkamp, E. H. F. van Asseldonk, and H. van der Kooij, "Design and Evaluation of the LOPES Exoskeleton Robot for Interactive Gait Rehabilitation", IEEE Transactions on Neural Systems and Rehabilitation Engineering, vol. 15, no. 3, pp. 379 386, 2007, ISSN: 07907915.

[17] P. Stegall, D. Zanotto, and S. K. Agrawal, "Variable damping force tunnel for gait training using ALEX III", Physiology and Behavior, vol. 2, no. 3, pp. 1495-1501, 2017, ISSN: 1873507X. DOI: 10.1016/j.physbeh.2017.03. 040 .

[18] B. Koopman, E. H. F. van Asseldonk, and H. van der Kooij, "Speeddependent reference joint trajectory generation for robotic gait support", Journal of Biomechanics, vol. 47, no. 6, pp. 1447-1458, 2014, ISSN: 18732380. DOI: $10.1016 / \mathrm{j} \cdot \mathrm{jbiomech.2014.01.037.}$

[19] S. F. Atashzar, M. Shahbazi, and R. V. Patel, "Haptics-enabled Interactive NeuroRehabilitation Mechatronics: Classification, Functionality, Challenges and Ongoing Research", Mechatronics, vol. 57, pp. 1-19, 2019, ISSN: 09574158. DOI: $10.1016 / \mathrm{j}$.mechatronics.2018.03.002.

[20] R. Colombo and V. Sanguineti, Assistive controllers and modalities for robot-aided neurorehabilitation. Elsevier Ltd., 2018, pp. 63-74, ISBN: 9780128119952. DOI: $10.1016 /$ b978-0-12-811995-2.00005-9.

[21] R. Gassert and V. Dietz, "Rehabilitation robots for the treatment of sensorimotor deficits: A neurophysiological perspective", Journal of NeuroEngineering and Rehabilitation, vol. 15, no. 1, pp. 1-15, 2018, ISSN: 17430003. DOI: $10.1186 / \mathrm{s} 12984-018-0383-\mathrm{x}$. 
[22] S. Maggioni, N. Reinert, L. Lünenburger, and A. Melendez-Calderon, "An Adaptive and Hybrid End-Point/Joint Impedance Controller for Lower Limb Exoskeletons", Frontiers in Robotics and AI, vol. 5, no. 104, 2018, ISSN: 1344-3542. DOI: $10.3389 /$ frobt.2018.00104.

[23] S. Srivastava, P. C. Kao, S. H. Kim, P. Stegall, D. Zanotto, J. S. Higginson, S. K. Agrawal, and J. P. Scholz, "Assist-as-Needed Robot-Aided Gait Training Improves Walking Function in Individuals Following Stroke", IEEE Transactions on Neural Systems and Rehabilitation Engineering, vol. 23, no. 6, pp. 956-963, 2015, ISSN: 15344320. DOI: 10.1109/ TNSRE . 2014 . 2360822 .

[24] D. Zanotto, P. Stegall, and S. K. Agrawal, "Adaptive assist-as-needed controller to improve gait symmetry in robot-assisted gait training", Proceedings - IEEE International Conference on Robotics and Automation, pp. 724-729, 2014, ISSN: 10504729. DOI: 10.1109/ICRA.2014.6906934.

[25] V. Dietz, R. Müller, and G. Colombo, "Locomotor activity in spinal man: significance of afferent input from joint and load receptors", Brain, vol. 125, no. 12 , pp. 2626-2634, 2002, ISSN: 0006-8950. DOI: 10.1093/brain/awf 273

[26] J. Hidler, D. Nichols, M. Pelliccio, K. Brady, D. D. Campbell, J. H. Kahn, and T. G. Hornby, "Multicenter randomized clinical trial evaluating the effectiveness of the Lokomat in subacute stroke", Neurorehabilitation and Neural Repair, vol. 23, no. 1, pp. 5-13, 2009, ISSN: 15459683. DOI: 10.1177/ 1545968308326632 .

[27] B. Husemann, F. Müller, C. Krewer, S. Heller, and E. Koenig, "Effects of locomotion training with assistance of a robot-driven gait orthosis in hemiparetic patients after stroke: A randomized controlled pilot study", Stroke, vol. 38, no. 2, pp. 349-354, 2007, ISSN: 00392499. DOI: $10.1161 / 01$. STR.0000254607.48765.cb.

[28] M. P. van Nunen, K. H. Gerrits, M. Konijnenbelt, T. W. Janssen, and A. de Haan, "Recovery of walking ability using a robotic device in subacute stroke patients: A randomized controlled study", Disability and Rehabilitation: Assistive Technology, vol. 10, no. 2, pp. 141-148, 2015, ISSN: 17483115. DOI: $10.3109 / 17483107.2013 .873489$.

[29] A. Esquenazi, S. Lee, A. Wikoff, A. Packel, T. Toczylowski, and J. Feeley, "A Comparison of Locomotor Therapy Interventions: Partial-Body WeightSupported Treadmill, Lokomat, and G-EO Training in People With Traumatic Brain Injury", $P M$ and $R$, vol. 9, no. 9, pp. 839-846, 2017, ISSN: 19341482. DOI: $10.1016 / \mathrm{j} \cdot \mathrm{pmrj} \cdot 2016.12 .010$.

[30] A. Furnari, R. S. Calabrò, M. C. De Cola, M. Bartolo, A. Castelli, A. Mapelli, G. Buttacchio, E. Farini, P. Bramanti, and R. Casale, "Roboticassisted gait training in Parkinson's disease: a three-month follow-up randomized clinical trial", International Journal of Neuroscience, vol. 127, no. 11, pp. 996-1004, 2017, ISSN: 15635279. DOI: 10.1080/00207454.2017. 1288623 . 
[31] S. Straudi, F. Manfredini, N. Lamberti, P. Zamboni, F. Bernardi, G. Marchetti, P. Pinton, M. Bonora, P. Secchiero, V. Tisato, S. Volpato, and N. Basaglia, "The effectiveness of Robot-Assisted Gait Training versus conventional therapy on mobility in severely disabled progressIve MultiplE sclerosis patients (RAGTIME): Study protocol for a randomized controlled trial", Trials, vol. 18, no. 1, pp. 1-12, 2017, ISSN: 17456215. DOI: 10.1186/s13063017-1838-2.

[32] C. Vaney, B. Gattlen, V. Lugon-Moulin, A. Meichtry, R. Hausammann, D. Foinant, A. M. Anchisi-Bellwald, C. Palaci, and R. Hilfiker, "Roboticassisted step training (Lokomat) not superior to equal intensity of overground rehabilitation in patients with multiple sclerosis", Neurorehabilitation and Neural Repair, vol. 26, no. 3, pp. 212-221, 2012, ISSN: 15459683. DOI: $10.1177 / 1545968311425923$

[33] K. P. Westlake and C. Patten, "Pilot study of Lokomat versus manualassisted treadmill training for locomotor recovery post-stroke", Journal of NeuroEngineering and Rehabilitation, vol. 6, no. 1, pp. 1-11, 2009, ISSN: 17430003. DOI: 10.1186/1743-0003-6-18.

[34] A. Mayr, M. Kofler, E. Quirbach, H. Matzak, K. Fröhlich, and L. Saltuari, "Prospective, blinded, randomized crossover study of gait rehabilitation in stroke patients using the Lokomat gait orthosis", Neurorehabilitation and Neural Repair, vol. 21, no. 4, pp. 307-314, 2007, ISSN: 15459683. DOI: $10.1177 / 1545968307300697$

[35] B. M. Fleerkotte, B. Koopman, J. H. Buurke, E. H. F. van Asseldonk, H. van der Kooij, and J. S. Rietman, "The effect of impedance-controlled robotic gait training on walking ability and quality in individuals with chronic incomplete spinal cord injury: An explorative study", Journal of NeuroEngineering and Rehabilitation, vol. 11, no. 26, 2014, ISSN: 17430003. DOI: 10.1186/1743-0003-11-26.

[36] I. J. Park, J.-H. Park, H. Y. Seong, J. S. H. You, S. J. Kim, J. H. Min, H. Y. Ko, and Y.-I. Shin, "Comparative Effects of Different Assistance Force During Robot-Assisted Gait Training on Locomotor Functions in Patients with Subacute Stroke", American Journal of Physical Medicine E Rehabilitation, vol. 98, no. 1, pp. 58-64, 2018, ISSN: 0894-9115. DOI: $10.1097 / \mathrm{phm} .0000000000001027$.

[37] J. H. Buurke, A. V. Nene, G. Kwakkel, V. Erren-Wolters, M. J. Ijzerman, and H. J. Hermens, "Recovery of gait after stroke: What changes?", Neurorehabilitation and Neural Repair, vol. 22, no. 6, pp. 676-683, 2008, ISSN: 15459683. DOI: $10.1177 / 1545968308317972$.

[38] L. Marchal-Crespo and R. Riener, Robot-assisted gait training. Elsevier Ltd., 2018, pp. 227-240, ISBN: 9780128119952. DOI: $10.1016 /$ b978-012-811995-2.00016-3. 
[39] J. Mehrholz, S. Thomas, C. Werner, J. Kugler, M. Pohl, and B. Elsner, "Electromechanical-assisted training for walking after stroke (Review)", Cochrane Database of Systematic Reviews, no. 5, 2017. DoI: 10.1002/ 14651858.CD006185.pub4.

[40] J. E. Cho, J. S. Yoo, K. E. Kim, S. T. Cho, W. S. Jang, K. H. Cho, and W. H. Lee, "Systematic Review of Appropriate Robotic Intervention for Gait Function in Subacute Stroke Patients", BioMed Research International, vol. 2018, 2018, ISSN: 23146141. DOI: 10.1155/2018/4085298

[41] M. F. Bruni, C. Melegari, M. C. De Cola, A. Bramanti, P. Bramanti, and R. S. Calabrò, "What does best evidence tell us about robotic gait rehabilitation in stroke patients: A systematic review and meta-analysis", Journal of Clinical Neuroscience, vol. 48, pp. 11-17, 2018, ISSN: 15322653. DOI: $10.1016 / j \cdot j o c n .2017 .10 .048$

[42] J. Mehrholz, L. A. Harvey, S. Thomas, and B. Elsner, "Is body-weight supported treadmill training or robotic-assisted gait training superior to overground gait training and other forms of physiotherapy in people with spinal cord injury? A systematic review", Spinal Cord, vol. 56, no. 4, p. 412, 2018, ISSN: 14765624. DOI: 10.1038/s41393-017-0059-6.

[43] C.-Y. Hsu, Y.-H. Cheng, C.-H. Lai, and Y.-N. Lin, "Clinical non-superiority of technology-assisted gait training with body weight support in patients with subacute stroke: A meta-analysis.", Annals of physical and rehabilitation medicine, 2019, ISSN: 1877-0665 (Electronic). DOI: 10.1016/j.rehab. 2019.09.009.

[44] S. C. Hayes, C. R. James Wilcox, H. S. Forbes White, and N. Vanicek, "The effects of robot assisted gait training on temporal-spatial characteristics of people with spinal cord injuries: A systematic review", The Journal of Spinal Cord Medicine, vol. 0, no. 0, pp. 1-15, 2018, ISSN: 1079-0268. DOI: 10.1080/10790268.2018.1426236

[45] B. Koopman, E. H. F. van Asseldonk, and H. van der Kooij, "Selective control of gait subtasks in robotic gait training: Foot clearance support in stroke survivors with a powered exoskeleton", Journal of NeuroEngineering and Rehabilitation, vol. 10, no. 1, 2013, ISSN: 17430003. DOI:10.1186/17430003-10-3.

[46] G. Chen, J. Ye, Q. Liu, L. Duan, W. Li, Z. Wu, and C. Wang, "Adaptive control strategy for gait rehabilitation robot to assist-when-needed", 2018 IEEE International Conference on Real-Time Computing and Robotics, RCAR 2018, pp. 538-543, 2018. DOI: 10.1109/RCAR.2018.8621706

[47] A. Duschau-Wicke, A. Caprez, and R. Riener, "Patient-cooperative control increases active participation of individuals with SCI during robot-aided gait training", Journal of NeuroEngineering and Rehabilitation, vol. 7, no. 1 , pp. 1-13, 2010, ISSN: 17430003. DOI: 10.1186/1743-0003-7-43 
[48] T. Aurich-Schuler and R. Labruyère, "An Increase in Kinematic Freedom in the Lokomat Is Related to the Ability to Elicit a Physiological Muscle Activity Pattern: A Secondary Data Analysis Investigating Differences Between Guidance Force, Path Control, and FreeD", Frontiers in Robotics and AI, vol. 6, pp. 1-9, 2019, ISSN: 2296-9144. DOI: 10.3389/frobt.2019.00109.

[49] L. Marchal-Crespo, P. Tsangaridis, D. Obwegeser, S. Maggioni, R. Riener, R. Group, M. Faculty, R. Group, S. Cord, M. Faculty, and C. Author, "Haptic Error Modulation Outperforms Visual Error Amplification when Learning a Modified Gait Pattern", Frontiers in Neuroscience, vol. 13, no. 61, 2019, ISSN: 1662-453X. DOI: 10.3389/fnins.2019.00061.

[50] C. Krishnan, D. Kotsapouikis, Y. Y. Dhaher, and W. Z. Rymer, "Reducing robotic guidance during robot-assisted gait training improves gait function: A case report on a stroke survivor", Archives of Physical Medicine and Rehabilitation, vol. 94, no. 6, pp. 1202-1206, 2013, ISSN: 00039993. DOI: 10.1016/j.apmr.2012.11.016.

[51] M. Maier, B. R. Ballester, and P. F. Verschure, "Principles of Neurorehabilitation After Stroke Based on Motor Learning and Brain Plasticity Mechanisms", Frontiers in Systems Neuroscience, vol. 13, no. 74, 2019, ISSN: 16625137. DOI: $10.3389 /$ fnsys.2019.00074

[52] C. Bayón, T. Martín-Lorenzo, B. Moral-Saiz, Ó. Ramírez, Á. PérezSomarriba, S. Lerma-Lara, I. Martínez, and E. Rocon, "A robot-based gait training therapy for pediatric population with cerebral palsy: Goal setting, proposal and preliminary clinical implementation", Journal of NeuroEngineering and Rehabilitation, vol. 15, no. 1, pp. 1-15, 2018, ISSN: 17430003. DOI: $10.1186 / \mathrm{s} 12984-018-0412-9$.

[53] G. Kwakkel, "Intensity of practice after stroke: More is better", Schweizer Archiv fur Neurologie und Psychiatrie, vol. 160, no. 7, pp. 295-298, 2009, ISSN: 02587661. DOI: 10.1080/09638280500534861.

[54] G. Kwakkel, R. Van Peppen, R. C. Wagenaar, S. W. Dauphinee, C. Richards, A. Ashburn, K. Miller, N. Lincoln, C. Partridge, I. Wellwood, and P. Langhorne, "Effects of augmented exercise therapy time after stroke: A meta-analysis", Stroke, vol. 35, no. 11, pp. 2529-2536, 2004, ISSN: 00392499. DOI: $10.1161 / 01$. STR.0000143153.76460.7d.

[55] J. M. Veerbeek, E. Van Wegen, R. Van Peppen, P. J. Van Der Wees, E. Hendriks, M. Rietberg, and G. Kwakkel, "What is the evidence for physical therapy poststroke? A systematic review and meta-analysis", PLoS ONE, vol. 9, no. 2, 2014, ISSN: 19326203. DOI: 10.1371/journal.pone.0087987

[56] K. van Kammen, A. M. Boonstra, L. H. van der Woude, C. Visscher, H. A. Reinders-Messelink, and R. den Otter, "Lokomat guided gait in hemiparetic stroke patients: the effects of training parameters on muscle activity and temporal symmetry", Disability and Rehabilitation, 2019, ISSN: 14645165. DOI: $10.1080 / 09638288.2019 .1579259$. 
[57] M. M. Mirbagheri, X. Niu, M. Kindig, and D. Varoqui, "The effects of locomotor training with a robotic-gait orthosis (Lokomat) on neuromuscular properties in persons with chronic SCI", Proceedings of the Annual International Conference of the IEEE Engineering in Medicine and Biology Society, EMBS, pp. 3854-3857, 2012, ISSN: 1557170X. DOI: 10.1109/EMBC. 2012.6346808.

[58] N. Sánchez, A. M. Acosta, R. Lopez-Rosado, A. H. A. Stienen, and J. P. A. Dewald, "Lower Extremity Motor Impairments in Ambulatory Chronic Hemiparetic Stroke: Evidence for Lower Extremity Weakness and Abnormal Muscle and Joint Torque Coupling Patterns", Neurorehabilitation and Neural Repair, vol. 31, no. 9, p. 154596831772 197, 2017, ISSN: 1545-9683. DOI: $10.1177 / 1545968317721974$.

[59] C. Shirota, E. H. F. van Asseldonk, Z. Matjačić, H. Vallery, P. Barralon, S. Maggioni, J. H. Buurke, and J. F. Veneman, "Robot-supported assessment of balance in standing and walking", Journal of NeuroEngineering and Rehabilitation, vol. 14, no. 1, pp. 1-19, 2017, ISSN: 17430003. DOI: 10.1186/s12984-017-0273-7.

[60] C. Bayón, "Design, Development and Evaluation of a Robotic Platform for Gait Rehabilitation and Training in Patients with Cerebral Palsy", PhD thesis, 2018. DOI: $10.1016 /$ j.robot.2016.12.015.

[61] G. Morone, S. Paolucci, A. Cherubini, D. De Angelis, V. Venturiero, P. Coiro, and M. Iosa, "Robot-assisted gait training for stroke patients: Current state of the art and perspectives of robotics", Neuropsychiatric Disease and Treatment, vol. 13, pp. 1303-1311, 2017, ISSN: 11782021. DOI: 10.2147/NDT.S114102.

[62] D. Zanotto, P. Stegall, and S. K. Agrawal, "ALEX III: A novel robotic platform with 12 DOFs for human gait training", in Proceedings - IEEE International Conference on Robotics and Automation, 2013, pp. 3914-3919, ISBN: 9781467356411. DOI: 10.1109/ICRA.2013.6631128.

[63] E. P. Washabaugh and C. Krishnan, "A wearable resistive robot facilitates locomotor adaptations during gait", Restorative Neurology and Neuroscience, vol. 36 , no. 2, pp. 215-223, 2018, ISSN: 18783627. DOI: 10.3233/ RNN-170782, 


\section{Acknowledgements}

This work would not have been possible without the help of many others. Here, I would like to thank everyone who contributed to this work and who supported me during the last years.

First of all, I would like to thank everyone who participated in my experiments. Thanks for your enthusiasm, patience, feedback, and the fun we had during the experiments. Without you this work would not have been possible!

Of course, only having great participants was not sufficient to finish this thesis. Another crucial ingredient were good supervision and collaboration. Herman and Edwin, I would like to thank you for this. Herman, thanks for your feedback and new ideas that further improved this work. Edwin, thanks for all your assistance! You were always there to help, not only with my thesis, but also my personal development.

Cristina and Ronald, thanks for being my paranymphs. Cristina, if you had not come to the Netherlands, this thesis would definitely not look like this. I enjoyed working together with you and also thanks for all your feedback on my papers and thesis. Ronald, thanks for the good collaboration on Chapter 2, and especially for helping me with all 'system identification questions'.

Thanks to all of my 'office mates' (especially Cristina, Guillaume, René and Ronald). I enjoyed sharing an office with you, and having random conversations when we needed a break from work. When we finally can work at the UT again we should go for another muffin (probably both the 'vreugde- en uithuilmuffinmeter' are already full again).

Next, I would thank all of my (other) colleagues from the Biomechanical Engineering group. I enjoyed working together with you, learning from you, or just having the lunch breaks and monthly drinks together. Our secretaries Lianne and Jeanine were always there to help with all kinds of questions. Our technicians, especially Nikolai, Quint and Wouter, 'saved' some experiments by repairing devices just before or even during my experiments. Gijs, thanks for teaching me how to use the LOPES and for helping out with all LOPES- and LOPER-related questions. Ander and Alfred, thanks for the good collaboration and all the work you did on Chapter 2. Celia, you helped a lot with the extra analyses of the clinical study. Unfortunately, due to time limitations, this work could not be included in this thesis anymore.

I also really enjoyed all the activities we did outside of work with some of the BE colleagues, such as playing board games, visiting German Christmas markets or doing sports together (running, bootcamp, mountain biking, ...). Maybe at 
some point we can finally go mountain biking together in Nijmegen? I heard that 'the best trails in the Netherlands' are in Nijmegen... When talking about sports, I should also not forget the BE/BSS futsal team and the 'UT kring' futsal group. Playing futsal with you during the lunch break or on Monday evening was always fun (and hopefully we can continue soon).

Jos, Karlijne and Tom, thanks for ensuring that we could continue using the LOPES. You were a great help when we had problems with the LOPES, thanks for all your efforts! Bertine, Brenda, Jaap, Jolanda and Martijn, thanks for your feedback on parts of my work (from the 'clinical point of view') and for helping with finding participants. Martijn, thanks again for all your help during the LOPES experiments, I enjoyed working together with you and the participants. Also, thanks to the whole user committee for the useful discussions and feedback during our meetings.

I supervised a number of students during my $\mathrm{PhD}$ and I enjoyed working together with them. Thanks for your enthusiasm and the work that you have done on LOPES II or other setups. A special thanks to Hilde who helped a lot with her work on Chapter 5. If you had not spent so many hours on finding suitable participants, we would probably still be searching for them now...

Last but not least, thanks to my friends and family for their support and all the nice activities that were needed to distract me from my thesis. Milou, thanks for the great cover design and all the 'cycling adventures'. Joran, thanks for supporting me during the last years and for always cheering me up. 


\section{Biography}

Simone Fricke was born in 1990 in Bremen (Germany). She studied Biomedical Engineering at the University of Twente (Enschede, The Netherlands) and received her bachelor degree in 2013 and her master degree in 2015 (both cum laude). In her master thesis she described the task-dependency of transcutaneous spinal direct current stimulation. During her master study she also performend an internship at the Center for Sensory-Motor Interaction (Aalborg, Denkmark) where she worked on research about soccer-induced central and peripheral fatigue and its effect on joint position sense in the ankle.

After her study, Simone joined the Department of Biome-

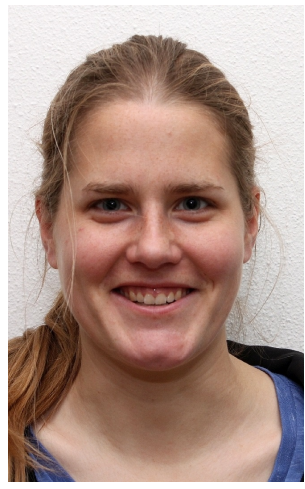
chanical Engineering at the University of Twente where she worked as a $\mathrm{PhD}$ candidate and performed research on robot-assisted gait training under the supervision of prof. dr. Herman van der Kooij and dr. Edwin van Asseldonk. Simone was involved in developing assessment tools and an automatically-tuned subtaskbased controller for the LOPES II gait trainer. Since April 2020, she is working as a Research Data Steward at the University of Twente. 


\section{Scientific contributions}

\section{Journal contributions}

- S.S. Fricke, C. Bayón, H. van der Kooij, E.H.F. van Asseldonk, Automatic versus manual tuning of robot-assisted gait training in people with neurological disorders, published in Journal of NeuroEngineering and Rehabilitation, 2020, 17(9)

- S.S. Fricke, H.J.G. Smits, C. Bayón, J.H. Buurke, H. van der Kooij, E.H.F. van Asseldonk, Effects of selectively assisting impaired subtasks of walking in chronic stroke survivors, submitted February 2020 (under review)

- S.S. Fricke, R.C. van 't Veld, A. Vallinas Prieto, H. van der Kooij, A.C. Schouten, E.H.F. van Asseldonk, Design and evaluation of a lower limb perturbator to estimate hip joint impedance during walking, submitted March 2020

\section{Peer-reviewed conference contributions}

- C. Bayón*, S.S. Fricke*, E. Rocon, H. van der Kooij, E.H.F. van Asseldonk, Performance-based adaptive assistance for diverse subtasks of walking in a robotic gait trainer: Description of a new controller and preliminary results, Proceedings of the IEEE RAS and EMBS International Conference on Biomedical Robotics and Biomechatronics, 2018, p. 414-419

*these authors contributed equally to this work 
ISBN: 978-90-365-5002-4

DOI: $10.3990 / 1.9789036550024$

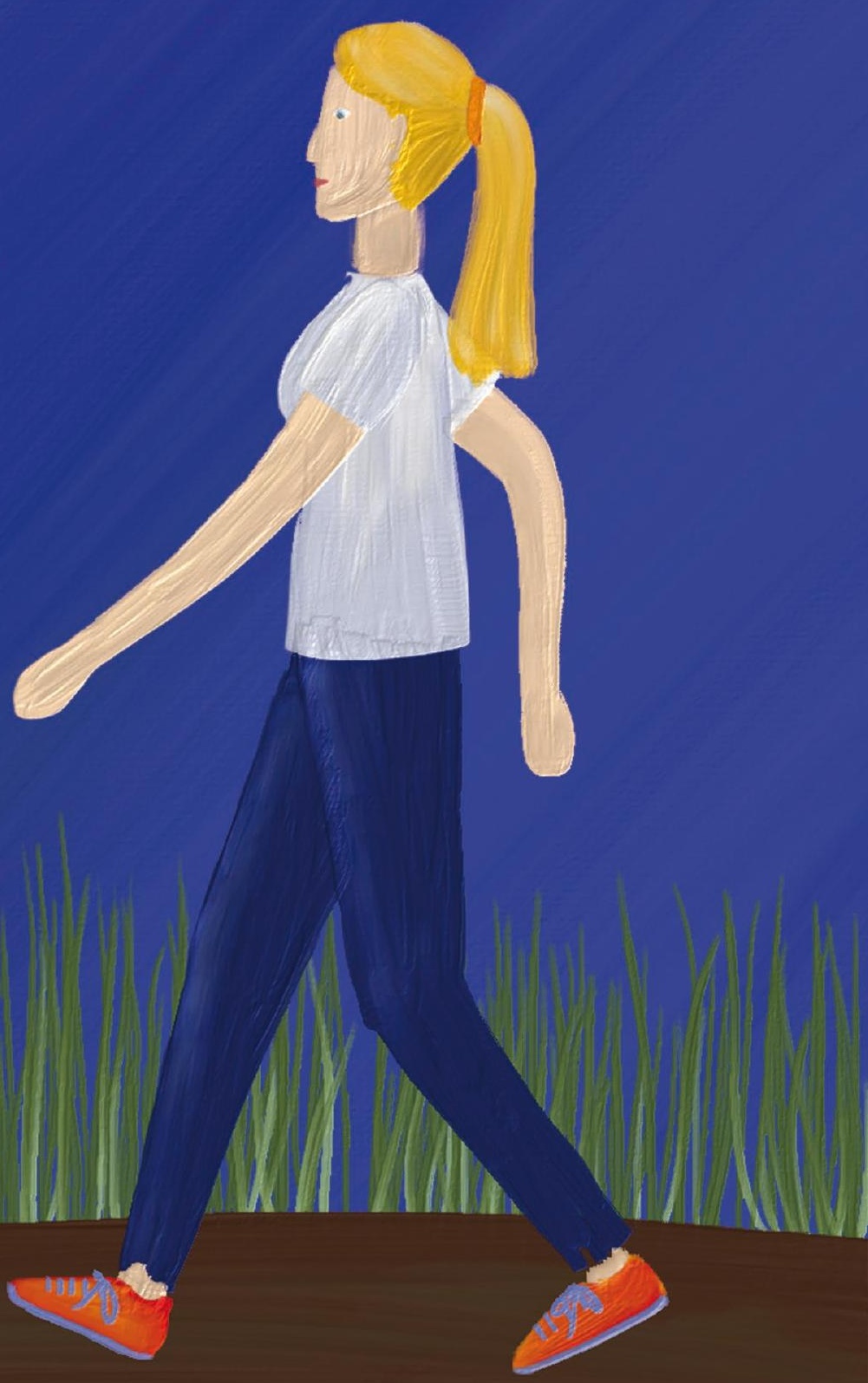

